\title{
Statistically Hiding Commitments and Statistical Zero-Knowledge Arguments from Any One-Way Function
}

\section{Citation}

Haitner, Iftach, Minh-Huyen Nguyen, Shien Jin Ong, Omer Reingold, and Salil Vadhan. 2009. "Statistically Hiding Commitments and Statistical Zero-Knowledge Arguments from Any OneWay Function." SIAM Journal on Computing 39, no. 3: 1153-1218.

\section{Published Version}

doi:10.1137/080725404

\section{Permanent link}

http://nrs.harvard.edu/urn-3:HUL.InstRepos:14123818

\section{Terms of Use}

This article was downloaded from Harvard University's DASH repository, and is made available under the terms and conditions applicable to Other Posted Material, as set forth at http:// nrs.harvard.edu/urn-3:HUL.InstRepos:dash.current.terms-of-use\#LAA

\section{Share Your Story}

The Harvard community has made this article openly available.

Please share how this access benefits you. Submit a story.

\section{Accessibility}




\title{
STATISTICALLY HIDING COMMITMENTS AND STATISTICAL ZERO-KNOWLEDGE ARGUMENTS FROM ANY ONE-WAY FUNCTION*
}

\author{
IFTACH HAITNER ${ }^{\dagger}$, MINH-HUYEN NGUYEN ${ }^{\ddagger}$, SHIEN JIN ONG ${ }^{\ddagger}$, OMER REINGOLD ${ }^{\S}$, \\ AND SALIL VADHAN ${ }^{\ddagger}$
}

\begin{abstract}
We give a construction of statistically hiding commitment schemes (those in which the hiding property holds against even computationally unbounded adversaries) under the minimal complexity assumption that one-way functions exist. Consequently, one-way functions suffice to give statistical zero-knowledge arguments for any NP statement (whereby even a computationally unbounded adversarial verifier learns nothing other than the fact that the assertion being proven is true, and no polynomial-time adversarial prover can convince the verifier of a false statement). These results resolve an open question posed by Naor et al. [J. Cryptology, 11 (1998), pp. 87-108].
\end{abstract}

Key words. cryptography, statistically hiding commitments, statistical zero-knowledge argument systems, one-way functions, interactive hashing

AMS subject classifications. 94A60, 68P25, 68Q99

DOI. $10.1137 / 080725404$

1. Introduction. As first discovered by Shannon [3] for the case of encryption, most interesting cryptographic tasks are impossible to achieve with absolute, information-theoretic security. Thus, modern cryptography aims to design protocols that are computationally intractable to break. Specifically, following Diffie and Hellman [4], this is typically done by showing that breaking the protocol is as hard as some intractable problem from complexity theory. Unfortunately, proving lower bounds of the sort needed seems beyond the reach of current techniques in complexity theory and indeed would require at least proving $\mathrm{P} \neq \mathrm{NP}$.

Given this state of affairs, research in the foundations of cryptography has aimed to design cryptographic protocols based on complexity assumptions that are as weak and general as possible. This project was enormously successful in the 1980s. In a beautiful sequence of works, it was shown that many cryptographic primitives, such as pseudorandom generators, pseudorandom functions, private-key encryption, authentication, digital signatures, (computationally hiding) commitment schemes, and (computational) zero-knowledge proofs, could be constructed from any one-way function $[5,6,7,8,9]$, and moreover this complexity assumption is minimal in the sense that each of these primitives (and indeed almost any cryptographic task) implies the

${ }^{*}$ Received by the editors November 5, 2007; accepted for publication (in revised form) February 24, 2009; published electronically September 2, 2009. Preliminary versions of this paper appeared as [1] and [2].

http://www.siam.org/journals/sicomp/39-3/72540.html

${ }^{\dagger}$ Microsoft Research, New England Campus, One Memorial Drive, Cambridge, MA 02142 (iftach@ microsoft.com). Most of this author's research was done while the author was at Weizmann Institute of Science, supported by grant 1300/05 from the Israel Science Foundation.

${ }^{\ddagger}$ School of Engineering and Applied Sciences, Harvard University, Cambridge, MA 02138 (mnguyen@eecs.harvard.edu, shienjin@eecs.harvard.edu, salil@eecs.harvard.edu). The research of these authors was supported by NSF grant CNS-0430336, ONR grant N00014-04-1-0478, and USIsrael BSF grant 2006060.

$\S$ Faculty of Mathematics and Computer Science, Weizmann Institute of Science, Rehovot, 76100, Israel (omer.reingold@weizmann.ac.il). The research of this author was supported by grant 1300/05 from the Israel Science Foundation. 
existence of one-way functions $[10,11]$. Moreover, it was shown that many of the remaining primitives, such as public-key encryption, collision-resistant hashing, and oblivious transfer, could not be reduced to the existence of one-way functions in a "black-box" manner [12, 13].

However, a few important primitives resisted classification into the above categories. That is, it was only known how to build these primitives from seemingly stronger assumptions than the existence of one-way functions, yet there was no blackbox separation between these primitives and one-way functions. In this work, we are interested in two such examples - statistically hiding commitment schemes and statistical zero-knowledge arguments for NP.

1.1. Statistically hiding commitments. A commitment scheme defines a two-stage interactive protocol between a sender $S$ and a receiver $R$; informally, after the commit stage, $S$ is bound to (at most) one value, which stays hidden from $R$, and in the reveal stage $R$ learns this value. The two security properties hinted at in this informal description are known as binding (namely, that $S$ is bound to at most one value after the commit stage) and hiding (namely, that $R$ does not learn the value to which $S$ commits before the reveal stage).

As with most cryptographic primitives, each of these security properties comes in two main flavors - computational security, whereby a polynomial-time adversary cannot violate the property except with negligible probability, and the stronger notion of statistical security, whereby even a computationally unbounded adversary cannot violate the property except with negligible probability. (An even stronger notion is that of perfect security, in which we do not even allow a negligible probability of breaking the scheme.) Naturally, statistical security, when achievable, is preferable to computational security. However, it can be shown that there do not exist commitment schemes that are simultaneously statistically hiding and statistically binding. Thus, at best we can hope for one of the two properties to be statistical and the other to be computational.

The complexity of statistically binding commitment schemes has been understood for a long time; they can be constructed from any one-way function $[8,5]$, and conversely, one-way functions are necessary for commitment schemes, even with both security properties computational [10]. In this work, however, we are interested in statistically hiding commitments, which have some advantages over statistically binding commitments. Specifically, when commitment schemes are used in constructing larger protocols, one typically needs the binding property to ensure the integrity of commitments that are opened during the protocol execution itself and the hiding property to ensure that the unopened commitments remain secret even after the protocol execution. Thus, for the binding property, we need only be concerned with the adversary's current resources, and thus it may be safe for this property to be computational. For the hiding property, however, we need to consider resources that the adversary may gain far into the future, and thus statistical security is preferable.

Some of the most important examples of cryptographic protocols based on commitments are the zero-knowledge protocols for proving membership in an arbitrary NP language $[9,14]$. In the protocol of [9], the hiding property of the commitment scheme translates to the zero-knowledge property of the protocol (i.e., the verifier learns nothing other than the fact that the assertion being proven is true), and the binding property of the commitment translates to the soundness property of the protocol, (i.e., the prover cannot convince the verifier of a false assertion). Thus, the existence of statistically hiding commitments implies that arbitrary NP statements 
can be proven with statistical zero knowledge and computational soundness; that is, every language in NP has a statistical zero-knowledge argument system [14, 15, 16].

Using statistically hiding commitments and the resulting statistical zero-knowledge arguments in known reductions [9, 17], one can actually transform any two-party protocol that is secure against passive (a.k.a. honest-but-curious) adversaries into one that is secure against malicious adversaries while preserving statistical security for one of the two parties.

Perfectly hiding commitment schemes and perfect zero-knowledge arguments for $\mathrm{NP}$ were first shown to exist based on specific number-theoretic assumptions [14, 15, $18,19,20]$ or, more generally, based on any collection of claw-free permutations [21, $22]$. The assumption for statistically hiding commitment schemes and statistical zeroknowledge arguments was reduced further to collision-resistant hash functions [23, 24]. Even though it seems intuitive that the computational binding property of statistically hiding commitments should be closely related to collision resistance, the beautiful work of Naor et al. [16] showed that actually any one-way permutation can be used to construct a perfectly hiding commitment scheme. Recently, Haitner et al. [25] reduced the assumption further by constructing statistically hiding commitments based on regular one-way functions with known preimage size, and more generally on one-way functions where the preimage sizes can be efficiently approximated. The question of whether an arbitrary, unstructured one-way function implies statistically hiding commitments or statistical zero-knowledge arguments for NP, however, was left open.

1.2. Our results. In this paper, we resolve the complexity of statistically hiding commitments.

THEOREM 1.1. If one-way functions exist, then statistically hiding commitment schemes exist.

By Impagliazzo and Luby [10], the existence of commitment schemes implies the existence of one-way functions, and thus the above result is tight.

As discussed above, combining Theorem 1.1 and standard constructions of zeroknowledge protocols from commitments (cf., $[9,14,15,16,26]$ ), we obtain our second main result.

THEOREM 1.2. If one-way functions exist, ${ }^{1}$ then every language in NP has a statistical zero-knowledge argument system.

The assumption that one-way functions exist also seems to be essentially minimal here: Ostrovsky and Wigderson $[27,11]$ showed that a zero-knowledge argument system for a hard-on-average problem implies the existence of one-way functions, and it follows from [26] that a zero-knowledge argument system for a language outside of $\mathrm{AM} \cap \mathrm{coAM}$ (or even outside SZKP) implies the existence of "nonuniform" one-way functions, where both the efficiency and security refer to polynomial-sized circuits (and security holds for infinitely many input lengths).

To avoid a lengthy detour into zero knowledge, we omit the formal definitions and proofs needed for Theorem 1.2 and instead refer the reader to [26], where our work plays a key role in proving unconditional results about zero-knowledge arguments.

1.3. Our techniques. We begin by using one-way functions to construct a variant of commitment schemes called 2-phase commitment schemes, recently introduced

\footnotetext{
${ }^{1}$ The standard definitions of zero knowledge and soundness are nonuniform notions of security, and thus this theorem requires assuming the existence of one-way functions that are secure even against nonuniform adversaries.
} 
by Nguyen and Vadhan [28]. ${ }^{2}$ We then use this 2-phase commitment scheme together with universal one-way hash functions (whose existence is also implied by the existence of one-way functions [7]) to construct the desired statistically hiding commitment scheme.

1.3.1. 2-phase commitments from any one-way function. 2-phase commitment schemes are commitment schemes with two phases, each consisting of a commit stage and a reveal stage. In the first phase, the sender commits to and reveals one value $v_{1}$, and subsequently, in the second phase, the sender commits to and reveals a second value $v_{2}$. We say that the 2 -phase commitment is hiding if both phases are hiding and say that it is 1-out-of-2-binding, symbolically written as $\left(\begin{array}{l}2 \\ 1\end{array}\right)$ binding, if the following holds: with high probability, the sender will be forced to reveal the correct committed value in at least one of the phases (but which of the two phases can be determined dynamically by the malicious sender?). More specifically, with high probability after the first-phase commit, there is a single value such that if the sender decommits to any other value, then the second commitment is guaranteed to be binding (in the standard sense).

Even though we draw upon [28] for the notion of 2-phase commitments, there are many differences between the contexts of the two works and their constructions of 2-phase commitments. In [28], the goal was to prove unconditional results about prover efficiency in zero-knowledge proofs (specifically, that one can transform zeroknowledge proofs with inefficient provers into ones with efficient provers). This was done by showing that every problem having a zero-knowledge proof has an "instancedependent" 2-phase commitment scheme, where the sender and receiver get an instance $x$ of the problem as auxiliary input and we only require hiding to hold when $x$ is a "yes instance" and binding when $x$ is a "no instance." Here, we are giving conditional results (assuming the existence of one-way functions) and are obtaining standard (as opposed to instance-dependent) 2-phase commitments. Moreover, the focus in [28] is on statistically binding 2-phase commitments; thus here we need to develop new formulations to work with the computational binding property.

Our initial construction, which gives a 2-phase commitment scheme satisfying a "weak hiding" property, is inspired by the construction of [28]. Indeed, the second phase in [28] was also introduced to deal with nonregular functions (corresponding to "nonflat distributions" in their setting), and our construction can be seen as applying the same idea to a variant of the protocol of [25]. However, in [28], this construction immediately gives a "strong hiding" property, whereas much of the technical work in the current paper comes from amplifying the "weak hiding" property we obtain into a strong one.

Like [28], another complication is that our initial construction does not provide a single 2-phase commitment scheme, but rather polynomially many schemes, one of which is weakly hiding and all of which are $\left(\begin{array}{l}2 \\ 1\end{array}\right)$-binding. After applying our amplification procedure and the transformation discussed below to each of these schemes, we obtain polynomially many standard commitments, at least one of which is statistically hiding and all of which are computationally binding; these can then be combined into a single statistically hiding commitment scheme using standard techniques.

\footnotetext{
${ }^{2}$ Using methods from [28], one can directly construct statistically zero-knowledge arguments for NP from 2-phase commitments and thereby prove Theorem 1.2. However, it is conceptually simpler to prove Theorem 1.1 and then deduce Theorem 1.2 using standard constructions.
} 


\subsubsection{From 1-out-of-2-binding commitments to standard commitments.} We would like to use a 2-phase commitment scheme to construct a (standard) commitment scheme. A naive attempt would simply have the receiver randomly choose, after the first commit phase, whether to stick with the first-phase commitment or to use the second-phase commitment as the actual commitment instead. The intuition is that since the commitment is $\left(\begin{array}{l}2 \\ 1\end{array}\right)$-binding, the sender cannot cheat in both phases together and thus the receiver would catch a cheating sender with probability one half (which we can then boost using standard techniques). The problem is, however, that the sender can decide in which phase he will cheat after knowing the receiver's choice. Hence, the sender can cheat successfully in both cases without violating the $\left(\begin{array}{l}2 \\ 1\end{array}\right)$-binding of the underlying protocol.

Our additional idea is to use a universal one-way hash function in order to force the sender to decide in which phase it is about to cheat before knowing the receiver's choice. Universal one-way hash functions are a relaxation of collision-resistant hash functions that were defined by Naor and Yung [23] and shown to be constructable from any one-way function by Rompel [7]. (See also [29].) We show that the above problem can be solved by having the sender provide a universal one-way hash of the secret he has committed to in the first phase. This turns out to (computationally) determine whether the first or second phase will be binding while leaving enough entropy in the first-phase secret to still achieve hiding.

1.4. Subsequent work. As the above suggests, our construction and its analysis are rather involved. Fortunately, a much simpler and more direct construction has recently been found [30]. Some of the techniques we develop here (such as those for working with collision probability as a measure of hiding in section 6) may nevertheless still be useful for other purposes.

1.5. Outline. We present the basic notation and definitions in section 2. As a warm-up, we present constructions of statistically hiding commitments based on one-way permutations in section 3 and from regular one-way functions with known preimage size in section 4 . In section 5 , we show how to construct 2 -phase commitments from regular one-way functions with unknown preimage size, and in section 6 , we extend it to any one-way function. Finally, in section 7, we present our transformation from 2-phase commitments to (standard) statistically hiding commitments.

\section{Definitions.}

2.1. Basic notation. If $X$ is a random variable taking values in a finite set $\mathcal{U}$, then we write $x \stackrel{\mathrm{R}}{\leftarrow} X$ to indicate that $x$ is selected according to $X$. If $S$ is a subset of $\mathcal{U}$, then $x \stackrel{\mathrm{R}}{\leftarrow} S$ means that $x$ is selected according to the uniform distribution on $S$. When the universe $\mathcal{U}$ is clear from context, we write $\mu(S)=|S| /|\mathcal{U}|$ to denote the density of $S$. We adopt the convention that when the same random variable occurs several times in an expression, all occurrences refer to a single sample. For example, $\operatorname{Pr}[f(X)=X]$ is defined to be the probability that when $x \stackrel{\mathrm{R}}{\leftarrow} X$, we have $f(x)=x$. We write $U_{n}$ to denote the random variable distributed uniformly over $\{0,1\}^{n}$. The support of a random variable $X$ is $\operatorname{Supp}(X)=\{x: \operatorname{Pr}[X=x]>0\}$. A random variable is flat if it is uniform over its support. If $X$ and $Y$ are random variables, then $X \otimes Y$ denotes the random variable obtained by taking independent random samples $x \stackrel{\mathrm{R}}{\leftarrow} X$ and $y \stackrel{\mathrm{R}}{\leftarrow} Y$ and outputting the pair $(x, y)$. We write $\otimes^{k} X$ to denote the random variable consisting of $k$ independent copies of $X$. For an event $E$, $\left.X\right|_{E}$ denotes the random variable $X$ conditioned on $E$. The statistical difference (also 
known as the variation distance) between random variables $X$ and $Y$ taking values in $\mathcal{U}$ is defined to be $\Delta(X, Y)=\max _{S \subset \mathcal{U}}|\operatorname{Pr}[X \in S]-\operatorname{Pr}[Y \in S]|$. We say that $X$ and $Y$ are $\varepsilon$-close if $\Delta(X, Y) \leq \varepsilon$.

A function $\varepsilon: \mathbb{N} \rightarrow[0,1]$ is called negligible if $\varepsilon(n)=n^{-\omega(1)}$. We let neg $(n)$ denote an arbitrary negligible function (i.e., when we say that $f(n)<\operatorname{neg}(n)$ we mean that there exists a negligible function $\varepsilon(n)$ such that for every $n, f(n)<\varepsilon(n))$. Likewise, $\operatorname{poly}(n)$ denotes an arbitrary function $f(n)=n^{O(1)}$.

For a probabilistic algorithm $A$, we write $A(x ; r)$ to denote the output of $A$ on input $x$ and coin tosses $r$. In this case, $A(x)$ is a random variable representing the output of $A$ for uniformly selected coin tosses. PPT (probabilistic polynomial-time) refers to probabilistic algorithms (i.e., Turing machines) that run in strict polynomial time. A nonuniform PPT algorithm is a pair $(A, \bar{z})$, where $\bar{z}=z_{1}, z_{2}, \ldots$ is an infinite sequence of strings in which $\left|z_{n}\right|=\operatorname{poly}(n)$, and $A$ is a PPT algorithm that receives pairs of inputs of the form $\left(x, z_{|x|}\right)$. (The string $z_{n}$ is called the advice string for $A$ for inputs of length $n$.) Nonuniform PPT algorithms are equivalent to (nonuniform) families of polynomial-sized Boolean circuits.

Two probability ensembles $\left\{X_{n}\right\}_{n \in \mathbb{N}}$ and $\left\{Y_{n}\right\}_{n \in \mathbb{N}}$ are computationally indistinguishable if for every PPT $D$, there exists a negligible function $\varepsilon$ such that for all $n \in \mathbb{N}$,

$$
\left|\operatorname{Pr}\left[D\left(1^{n}, X_{n}\right)=1\right]-\operatorname{Pr}\left[D\left(1^{n}, Y_{n}\right)=1\right]\right| \leq \varepsilon(|x|) .
$$

Similarly, we say that $\left\{X_{n}\right\}$ and $\left\{Y_{n}\right\}$ are statistically indistinguishable if the above is required for all functions $D$ (instead of only PPT $D$ 's). Equivalently, $\left\{X_{n}\right\}$ and $\left\{Y_{n}\right\}$ are statistically indistinguishable if $X_{n}$ and $Y_{n}$ are $\varepsilon(n)$-close for some negligible function $\varepsilon$ and all $n \in \mathbb{N}$.

An interactive protocol $(A, B)$ consists of two algorithms that compute the nextmessage functions of the (honest) parties in the protocol. Specifically, $A\left(x, a, \alpha_{1}, \ldots\right.$, $\left.\alpha_{k} ; r\right)$ denotes the next message $\alpha_{k+1}$ sent by party $A$ when the common input is $x$, $A$ 's auxiliary input is $a, A$ 's coin tosses are $r$, and the messages exchanged so far are $\alpha_{1}, \ldots, \alpha_{k}$. There is a special message, halt, which immediately halts the interaction, at which time each party can compute one more message, which is their private output. Sometimes we will refer to protocols with a joint output, which is required to be a deterministic polynomial-time function of just the common input and transcript of messages exchanged (and not the parties' auxiliary inputs or private coin tosses). We say that party $A$ (resp., $B$ ) is probabilistic polynomial-time (PPT) if its next-message function can be computed in polynomial time (in $|x|+|a|+\left|\alpha_{1}\right|+\cdots+\left|\alpha_{k}\right|$ ).

For an interactive protocol $(A, B)$, we write $(A(a), B(b))(x)$ to denote the random process obtained by having $A$ and $B$ interact on common input $x$, with (private) auxiliary inputs $a$ and $b$ to $A$ and $B$, respectively (if any), and with independent random coin tosses for $A$ and $B$. We call $(A, B)$ polynomially bounded if there is a polynomial $p$ such that for all $x, a, b$, the total length of all messages exchanged in $(A(a), B(b))(x)$ is at most $p(|x|)$ with probability 1 . Moreover, if $B^{*}$ is any interactive algorithm, then $A$ will immediately halt in $\left(A(a), B^{*}(b)\right)(x)$ if the total length of the messages ever exceeds $p(|x|)$; we have the analogous requirement for $B$ interacting with any $A^{*}$. We call a protocol $(A, B)$ a polynomial time if $(A, B)$ is polynomially bounded and both $A$ and $B$ are PPT.

The number of rounds in an execution of the protocol is the total number of messages exchanged between $A$ and $B$, not including the final accept/reject message. We call the protocol $(A, B)$ public coin for $A$ (resp., $B$ ) if all the messages sent by $A$ 
(resp., $B$ ) are simply the output of its coin tosses (independent of the history), except for the final halt message and $A$ 's (resp., $B$ 's) private output, which is computed as a deterministic function of the transcript.

We associate several random variables with the interaction $(A(a), B(b))(x)$. The private output of $A$ is denoted by output $A(A(a), B(b))(x)$, and view $A(A(a), B(b))(x)$ denotes $A$ 's view of the interaction; i.e., its values are transcripts $\left(\gamma_{1}, \gamma_{2}, \ldots, \gamma_{t} ; r\right)$, where the $\gamma_{i}$ 's are all the messages exchanged and $r$ is A's coin tosses. Similarly, output $_{B}(A(a), B(b))(x)$ and view $B(A(a), B(b))$ denote $B$ 's private output and view, respectively. The joint output, if any, is denoted by output $(A(a), B(b))(x)$.

2.2. One-way functions. The most basic primitive of modern cryptography is a one-way function, which is a function that is easy to compute but hard to invert.

Definition 2.1. Let $s: \mathbb{N} \rightarrow \mathbb{N}$ be any function. A function $f:\{0,1\}^{*} \rightarrow$ $\{0,1\}^{*}$ is an $s(n)$-secure one-way function, or equivalently has security $s(n)$, if $f$ is computable in polynomial time and for every PPT A,

$$
\operatorname{Pr}_{y \leftarrow\{0,1\}^{n}}\left[A\left(1^{n}, f(y)\right) \in f^{-1}(f(y))\right]<1 / s(n)
$$

for all sufficiently large $n$. Function $f$ is a one-way function if $f$ is $s(n)$-secure for every polynomial s. If the above holds also for nonuniform PPT A, we say that $f$ is nonuniformly secure.

One-way function $f$ is a regular one-way function if there exists a function $g: \mathbb{N} \rightarrow$ $\mathbb{N}$ such that $\forall z \in \operatorname{Supp}\left(f\left(U_{n}\right)\right),\left|\left\{y \in\{0,1\}^{n}: f(y)=z\right\}\right|=g(n) ; g(n)$ is called the preimage size of $f$. We say that $f$ is known-regular if $g(n)$ can be computed in time $\operatorname{poly}(n)$.

Without loss of generality, we can consider only one-way functions (regular or nonregular) that are length-preserving, that is, for all $y \in\{0,1\}^{*},|f(y)|=|y|$. This is because general one-way functions can be converted into ones that are lengthpreserving (cf., [31, p. 39]).

2.3. Commitment schemes. Another basic primitive of modern cryptography is a (bit) commitment scheme, which is a two-stage protocol between a sender and a receiver. In the first stage, called the commit stage, the sender commits to a private bit $b$. In the second stage, called the reveal stage, the sender reveals $b$ and proves that it was the bit to which she committed in the first stage. We require two properties of commitment schemes. The hiding property says that the receiver learns nothing about $b$ in the commit stage. The binding property says that after the commit stage, the sender is bound to a particular value of $b$; that is, she cannot successfully open the commitment to two different bits in the reveal stage.

Definition 2.2. A commitment scheme is a polynomial-time interactive protocol Com $=(S, R)$ with the following properties:

1. Scheme Com proceeds in two stages: a commit stage and a reveal stage. In both stages, the sender $S$ and the receiver $R$ receive a security parameter $1^{n}$ as common input.

2. At the beginning of the commit stage, sender $S$ receives a private input $b \in$ $\{0,1\}$, which denotes the bit that $S$ is supposed to commit to. The commitment stage results in a joint output, which we call the commitment $c=$ output $\left((S(b), R)\left(1^{n}\right)\right)$, and a private output for $S$, which we call the decommitment string $d=$ output $_{S}(S(b), R)\left(1^{n}\right)$. Without loss of generality, $c$ can be taken to be the full transcript of the interaction between $S$ and $R$, and $d$ to be the private coin tosses of $S$. 
3. In the reveal stage, sender $S$ sends the pair $(b, d)$, where $d$ is the decommitment string for bit $b$. Receiver $R$ accepts or rejects based on $b, d$, and $c$.

4. $R$ will always accept (with probability 1 ) if both sender $S$ and receiver $R$ follow their prescribed strategy.

A commitment scheme is public coin if all messages sent by the receiver are independent random coins.

Next, we define the hiding and binding properties of commitment schemes.

Definition 2.3. Commitment scheme Com $=(S, R)$ is statistically (resp., computationally) hiding if for every (resp., PPT) $R^{*}$, the ensembles $\left\{\operatorname{view}_{R^{*}}(S(0)\right.$, $\left.\left.R^{*}\right)\left(1^{n}\right)\right\}_{n \in \mathbb{N}}$ and $\left\{\operatorname{view}_{R^{*}}\left(S(1), R^{*}\right)\left(1^{n}\right)\right\}_{n \in \mathbb{N}}$ are statistically (resp., computationally) indistinguishable, where view $R^{*}\left(S(b), R^{*}\right)$ denotes the view of $R^{*}$ in the commit stage interacting with $S(b)$.

Definition 2.4. Commitment scheme Com $=(S, R)$ is statistically (resp., computationally) binding if for every (resp., PPT) $S^{*}$, there exists a negligible function $\varepsilon$ such that the malicious sender $S^{*}$ succeeds in the following game with probability at most $\varepsilon(n)$ :

On security parameter $1^{n}, S^{*}$ interacts with $R$ in the commit stage obtaining commitment $c$. Then $S^{*}$ outputs pairs $\left(0, d_{0}\right)$ and $\left(1, d_{1}\right)$ and succeeds if in the reveal stage $R\left(0, d_{0}, c\right)=R\left(1, d_{1}, c\right)=$ accept.

If the above holds for every nonuniform PPT $S^{*}$, we say that Com is computationally binding with nonuniform security.

Constructing commitment schemes based on any one-way function. Naor [8] constructed commitment schemes that are computationally hiding and statistically binding from any pseudorandom generator, which in turn can be based on any one-way function [5]. The main result of this paper, Theorem 1.1, shows that commitment schemes that are statistically hiding and computationally binding can be based on any one-way function.

3. Statistically hiding commitments from one-way permutations. Consider a one-way permutation $f:\{0,1\}^{n} \rightarrow\{0,1\}^{n}$. Naor et al. [16] obtained a statistically hiding commitment scheme based on $f$ by using a protocol called interactive hashing as a subroutine. Our agenda for this section is as follows: we will first informally describe interactive hashing and state the two main properties that we want from it; then, in section 3.1 we give an informal description of the Naor et al. [16] scheme, henceforth called the NOVY commitment scheme; and finally, in section 3.2, we give a formal definition of interactive hashing and a protocol satisfying that definition.

Interactive hashing is a protocol between a sender $S_{\mathrm{IH}}$ and a receiver $R_{\mathrm{IH}}$. The sender begins with a private input $z$, and at the end both parties output $z_{0}$ and $z_{1}$ such that $z \in\left\{z_{0}, z_{1}\right\}$. Informally, an interactive hashing protocol has the following two properties:

1. Hiding: If the sender's private input $z$ is uniformly random, then every receiver, even computationally unbounded malicious ones, fails to learn which of $z_{0}$ or $z_{1}$ equals to $z$.

2. Binding: The sender, including PPT malicious ones, can only "control" the value of at most one of the two outputs; the value of the other output should be essentially uniformly distributed.

3.1. The NOVY commitment scheme. Using an interactive hashing protocol as a subroutine, Naor et al. [16] constructed the following statistically hiding commitment scheme. 
1. $S$ chooses a uniform $x \leftarrow\{0,1\}^{n}$ and computes $z=f(x)$.

2. $S$ and $R$ engage in an interactive hashing protocol using $z$ as $S$ 's private input. Let $z_{0}$ and $z_{1}$ be the common outputs, and let $z=z_{d}$ for some $d \in\{0,1\}$.

3. To commit to bit $b, S$ sends $c=b \oplus d$ to $R$.

4. To decommit, $S$ sends $b, d$, and $x$ to $R$. $R$ verifies the decommitment by checking if $c=b \oplus d$ and $z_{d}=f(x)$.

Let us informally argue why the above scheme constitutes a statistically hiding and computationally binding commitment. First, we argue its hiding property. We have mentioned that $z$ is uniform in $\{0,1\}^{n}$ because $f$ is a permutation and $x$ is chosen uniformly in $\{0,1\}^{n}$. By the hiding property of interactive hashing, even a computationally unbounded malicious receiver does not know if $z=z_{0}$ or $z=z_{1}$, or equivalently, it does not know if $d=0$ or $d=1$. Therefore, the scheme is statistically hiding. Next, we argue its binding property. By the binding property of interactive hashing, at least one of the outputs, say, $z_{\alpha}$, is uniform in $\{0,1\}^{n}$ and outside the sender's control. Therefore, if the sender is able to decommit to both 0 and 1 , it must find a preimage of $z_{\alpha}$. This is equivalent to finding a preimage of $f\left(U_{n}\right)$, and this task is computationally infeasible since $f$ is a one-way permutation. Hence, the scheme is computationally binding.

3.2. Interactive hashing. Interactive hashing was introduced by Ostrovsky, Venkatesan, and Yung [32] in the context of oblivious transfer protocols. As mentioned above, it was applied to the construction of statistically hiding commitments by Naor et al. [16], and it will also prove to be a powerful and useful tool in our result. For our application, we will need the sender to commit to multiple bits in one execution of interactive hashing. Consequently, we extend the notion of interactive hashing to allow multiple outputs (instead of just two output strings). Since the number of outputs could be possibly superpolynomial, we succinctly describe the set of outputs as the image of a polynomial-sized circuit $C:\{0,1\}^{k} \rightarrow\{0,1\}^{q}$, where $k$ and $q$ are polynomially related to the security parameter. (We will not actually need superpolynomially many outputs in this paper but use this more general formulation because it may be useful in future work.)

In addition to allowing for multiple outputs, our application of interaction hashing also requires a more refined notion of computational binding than the one provided by Naor et al. [16]. ${ }^{3}$ It is for this reason that we consider the notion of what it means to be a witness for a given relation $W$ as follows: For a relation $W$, define the set of witnesses for $z$ as $W_{z}=\{x: W(z, x)=1\}$, and we naturally refer to any $x \in W_{z}$ as a witness for $z$. (A natural choice, utilized in the analysis of the NOVY commitment scheme described above, is $W=\{(z, x): f(x)=z\}$ for a one-way function/permutation $f$.)

Definition 3.1. An interactive hashing protocol with multiple outputs is a polynomial-time protocol $\left(S_{\mathrm{IH}}, R_{\mathrm{IH}}\right)$ where both parties receive common inputs $\left(1^{q}, 1^{k}\right)$ and $S_{\mathrm{IH}}$ receives a private input $z \in\{0,1\}^{q}$. At the end of the interaction, the common output is a (polynomial-sized) circuit $C:\{0,1\}^{k} \rightarrow\{0,1\}^{q}$, and the private output of $S_{\mathrm{IH}}$ is a string $d \in\{0,1\}^{k}$. We call $q$ the input length and $k$ the output length. The protocol $\left(S_{\mathrm{IH}}, R_{\mathrm{IH}}\right)$ has to satisfy the following security properties.

1. Correctness: For all $R^{*}$ and all $z \in\{0,1\}^{q}$, it is the case that $C(d)=z$, where $C=\left(S_{\mathrm{IH}}(z), R^{*}\right)\left(1^{q}, 1^{k}\right)$ is the common output, and $d=$ output $_{\mathrm{IH}_{\mathrm{H}}}\left(S_{\mathrm{IH}}(z), R^{*}\right)$

\footnotetext{
${ }^{3}$ Although the notion of interactive hashing was introduced by Ostrovsky, Venkatesan, and Yung [32], it was Naor et al. [16] who proved a computational binding property of interactive hashing that allows for its application to statistically hiding commitments based on any one-way permutation.
} 
is the private output of $S_{\mathrm{IH}}{ }^{4}$

2. Hiding: For all $R^{*}$, random variables $(V, D)$ and $\left(V, U_{k}\right)$ are identically distributed, where the view of receiver $R^{*}$ is $V=\operatorname{view}_{R^{*}}\left(S_{\mathrm{IH}}\left(U_{q}\right), R^{*}\right)$, and the private output of $S_{\mathrm{IH}}$ is $D=$ output $_{S_{\mathrm{IH}}}\left(S_{\mathrm{IH}}\left(U_{q}\right), R^{*}\right)$.

3. Binding: There exists an oracle PPT algorithm A such that for every adversary $S^{*}$ and any relation $W$, denoting the common output as $C=$ $\left(S^{*}, R_{\mathrm{IH}}\right)\left(1^{q}, 1^{k}\right)$, and private output of $S^{*}$ as $\left(\left(x_{0}, d_{0}\right),\left(x_{1}, d_{1}\right)\right)=$ output $_{S^{*}}\left(S^{*}, R_{\mathrm{IH}}\right)$, if it is the case that

$$
\operatorname{Pr}\left[x_{0} \in W_{C\left(d_{0}\right)} \wedge x_{1} \in W_{C\left(d_{1}\right)} \wedge d_{0} \neq d_{1}\right]>\varepsilon,
$$

where the above probability is over the coins of $R_{\mathrm{IH}}$ and $S^{*}$, then it is also the case that

$$
\operatorname{Pr}_{z \leftarrow\{0,1\}^{q}}\left[A^{S^{*}}\left(z, 1^{q}, 1^{k}, \varepsilon\right) \in W_{z}\right]>2^{-k} \cdot(\varepsilon / q)^{O(1)} .
$$

Remark 3.2. We make three remarks regarding Definition 3.1.

1. The security requirements should hold for computationally unbounded $R^{*}$ (for correctness and hiding) and computationally unbounded $S^{*}$. In addition, the relation $W$ need not be polynomial-time computable.

2. To simplify notation, we often write $A^{S^{*}}(z)$, or even $A(z)$, to denote $A^{S^{*}}\left(z, 1^{q}\right.$, $\left.1^{k}, \varepsilon\right)$.

3. Although the private output of the honest sender $S_{\mathrm{IH}}$ is always a string $d$, the private output of the cheating sender $S^{*}$ is arbitrary; hence, we can assume without loss of generality that $S^{*}$ breaks binding by producing two pairs of strings $\left(x_{0}, d_{0}\right)$ and $\left(x_{1}, d_{1}\right)$.

The interactive hashing protocol given in $[32,16]$, henceforth called the NOVY Interactive Hashing protocol, satisfies Definition 3.1 with $k=1$. To obtain an interactive hashing protocol with multiple outputs (i.e., the case when $k>1$ ), we simply end the NOVY Interactive Hashing protocol $k-1$ rounds earlier.

Protocol 3.3. Interactive hashing with multiple outputs $\left(S_{\mathrm{IH}}, R_{\mathrm{IH}}\right)$.

Inputs:

1. Input length $1^{q}$ and output length $1^{k}$, both given as common input.

2. String $z \in\{0,1\}^{q}$, given as private input to sender $S_{\mathrm{IH}}$.

Protocol:

$R_{\mathrm{IH}}$ : Select $h_{0}, h_{1}, \ldots, h_{q-k-1}$ such that each $h_{i}$ is a random vector over $\mathrm{GF}[2]$ that is outside the span of $\left\{h_{0}, h_{1}, \ldots, h_{i-1}\right\}$.

For $j=0, \ldots, q-k-1$, do the following:

$R_{\mathrm{IH}} \rightarrow S_{\mathrm{IH}}:$ Send $h_{j}$.

$S_{\mathrm{IH}} \rightarrow R_{\mathrm{IH}}:$ Send $c_{j}=\left\langle h_{j}, z\right\rangle$.

Output:

- Common output is a circuit $C:\{0,1\}^{k} \rightarrow\{0,1\}^{q}$ computing an injective affine transformation whose image is $\left\{z:\left\langle h_{j}, z\right\rangle=c_{j} \forall j=0, \ldots, q-k-1\right\}$.

- Private output of $S_{\mathrm{IH}}$ is a string $d \in\{0,1\}^{k}$ such that $C(d)=z$.

THEOREM 3.4. There exists an interactive hashing protocol with multiple outputs, namely, Protocol 3.3.

\footnotetext{
${ }^{4}$ The correctness property of protocols is typically defined for honest parties; in our setting this would be $S_{\mathrm{IH}}$ and $R_{\mathrm{IH}}$. In our applications, however, it is convenient to have a stronger correctness property that also holds against malicious receivers $R^{*}$.
} 
The correctness of Protocol 3.3 is easy to see. Hence, we divide the proof of this theorem into lemmas establishing the hiding and binding properties of Protocol 3.3.

Lemma 3.5. Protocol 3.3 satisfies the hiding property of Definition 3.1. In other words, letting interactive hashing $\left(S_{\mathrm{IH}}, R_{\mathrm{IH}}\right)$ be as in Protocol 3.3, we have for all $R^{*}$ that $(V, D)$ is distributed identically to $\left(V, U_{k}\right)$, where $V=\operatorname{view}_{R^{*}}\left(S_{\mathrm{IH}}\left(U_{q}\right), R^{*}\right)$ is the view of receiver $R^{*}$, and $D=$ output $_{S_{\mathrm{IH}}}\left(S_{\mathrm{IH}}\left(U_{q}\right), R^{*}\right)$ is the private output of $S_{\mathrm{IH}}$.

Proof. The view of any $R^{*}$ will be the hash functions $h_{0}, h_{1}, \ldots, h_{q-k-1}$ together with $S_{\mathrm{IH}}$ 's responses $c_{0}, c_{1}, \ldots, c_{q-k-1}$. Conditioned on such a view, the sender's private input $Z=U_{q}$ is uniformly distributed in the $k$-dimensional affine subspace $\left\{z:\left\langle h_{j}, z\right\rangle=c_{j} \forall j\right\}$, and hence the value $D$ s.t. $C(D)=Z$ is uniformly distributed in $\{0,1\}^{k}$.

Lemma 3.6. Protocol 3.3 satisfies the binding property of Definition 3.1. That is, letting interactive hashing $\left(S_{\mathrm{IH}}, R_{\mathrm{IH}}\right)$ be as in Protocol 3.3, there exists an oracle PPT algorithm A such that

for every $S^{*}$ and any relation $W$, denoting the common output as $C=$ $\left(S^{*}, R_{\mathrm{H}}\right)\left(1^{q}, 1^{k}\right)$, and private output of $S^{*}$ as $\left(\left(x_{0}, d_{0}\right),\left(x_{1}, d_{1}\right)\right)=$ output $_{S^{*}}\left(S^{*}, R_{\mathrm{IH}}\right)$, if it is the case that

$$
\operatorname{Pr}\left[x_{0} \in W_{C\left(d_{0}\right)} \wedge x_{1} \in W_{C\left(d_{1}\right)} \wedge d_{0} \neq d_{1} \in\{0,1\}^{k}\right]>\varepsilon,
$$

where the above probability is over the coin tosses of $R_{\mathrm{IH}}$ and $S^{*}$, then it is also the case that

$$
\operatorname{Pr}_{z \leftarrow\{0,1\}^{q}}\left[A^{S^{*}}\left(z, 1^{q}, 1^{k}, \varepsilon\right) \in W_{z}\right]=\Omega\left(\varepsilon^{2} q^{-8} 2^{-k}\right) .
$$

Proof. Note that $C\left(d_{0}\right)$ and $C\left(d_{1}\right)$ are two distinct elements in $\{0,1\}^{q}$ and that both elements are consistent with the transcript of the protocol; i.e., an honest $S_{\mathrm{IH}}$ getting each of these elements as input would have acted in the same way as $S^{*}$ does in the interaction. Thus, we are in the setting of the recent interactive hashing theorem presented by Haitner and Reingold [33], and the proof follows by [33, Theorem $3.9 .^{5}$

3.2.1. Information-theoretic bounds. We think of the string $d$ as a $k$-bit string commitment associated to one of the $2^{k}$ outputs strings, namely, $z=C(d)$, and a witness $x \in W_{z}=W_{C(d)}$ as a decommitment to $d$. Intuitively, the knowledge of $x$ gives the sender the ability to decommit to $d$. The binding property, read in its contrapositive, says that if it is hard to find a witness for a uniformly random string $z$, then it is hard for a sender to successfully decommit to two different values. Note that this hypothesis - that it is hard to find a witness for a uniformly random $z$ - says that for every PPT $A$, there is a negligible set $T_{A}$ of $z$ 's on which $A$ succeeds in finding a witness. In general, the set $T_{A}$ may depend on the adversary $A$. In several places, however, we will need only the special case of a static set as captured in the following lemma.

LEMma 3.7 (binding for static sets). For any protocol $\left(S_{\mathrm{IH}}, R_{\mathrm{IH}}\right)$ satisfying the binding condition of Definition 3.1, the following holds: For all $S^{*}$ and any set $T \subseteq$

\footnotetext{
${ }^{5}$ Actually, [33, Theorem 3.9] directly applies to the case that the $h_{i}$ 's are uniformly random vectors rather than being constrained to be linearly independent as in Protocol 3.3. However, up to $q$ uniformly random $q$-dimensional vectors will be linearly independent with at least constant probability. Thus, if $S^{*}$ breaks Protocol 3.3 with probability greater than $\varepsilon$, then it also breaks the version with uniformly random vectors with probability $\Omega(\varepsilon)$, and [33, Theorem 3.9] applies.
} 
$\{0,1\}^{q}$, denoting the common output as $C=\left(S^{*}, R_{\mathrm{IH}}\right)\left(1^{q}, 1^{k}\right)$, we have

$$
\operatorname{Pr}\left[\exists d_{0} \neq d_{1} \text { such that } C\left(d_{0}\right), C\left(d_{1}\right) \in T\right]<O\left(q^{4}\right) \cdot\left(\mu(T) \cdot 2^{k}\right)^{1 / 2},
$$

where the above probability is taken over the coins of $S^{*}$ and $R_{\mathrm{IH}}$ and $\mu(T) \stackrel{\text { def }}{=}|T| / 2^{q}$ denotes the density of $T$.

Setting $k=1$ in the above lemma gives an information-theoretic bound of the NOVY Interactive Hashing protocol; information-theoretic bounds on NOVY Interactive Hashing were studied in the context of memory-bounded oblivious transfer $[34,35,36]$. Our bound is not tight but suffices for our applications. For tighter bounds, we refer the reader to $[34,36]$, or for a constant-round interactive hashing protocol that is binding for static sets, we refer the reader to [35].

Compare the bound of Lemma 3.7 to the case where the adversarial sender $S^{*}$ had control of only one output string. This means that the rest of the $2^{k}-1$ output strings are distributed uniformly on $\{0,1\}^{q}$, and hence the bound would be roughly $\mu(T) \cdot\left(2^{k}-1\right)$. The reason for this is that $S^{*}$ will make the string that it controls lie in $T$, and the probability that at least one of the rest of the $2^{k}-1$ strings lie in $T$ is at most $\mu(T) \cdot\left(2^{k}-1\right)$, by a union bound. The above bound is almost as good, and, in particular, if $\mu(T)$ is negligible and $k$ logarithmic, both probabilities are negligible.

Proof of Lemma 3.7. Define the relation $W=\{(a, b): a \in T\}$, that is, $W(a, b)=1$ if $a \in T$ (for all values of $b$ ), and 0 if $a \notin T$ (no matter what the value of $b$ is). Suppose there exists an $S^{*}$ that with probability $\varepsilon$ produces two elements $d_{0} \neq d_{1}$ such that both $C\left(d_{0}\right), C\left(d_{1}\right) \in T$. Then, by Lemma 3.6 , there will be a procedure that is given a random $z \leftarrow\{0,1\}^{q}$ and makes $z \in T$ with probability $p=\Omega\left(2^{-k} \cdot \varepsilon^{2} / q^{8}\right)$. Since $T$ is a fixed set, it must be the case that $p \leq \mu(T)$. This implies that $\varepsilon=$ $O\left(q^{4}\right) \cdot\left(\mu(T) \cdot 2^{k}\right)^{1 / 2}$.

4. Statistically hiding commitments from known-regular one-way functions. Our first hurdle is to relax the permutation structure of $f$ to just assuming that $f$ is a regular one-way function with known preimage size of, say, $2^{n-t}$ for some known value of $t \in\{1,2, \ldots, n\}$. This is the setting considered by Haitner et al. [25], and we review ideas from their construction in this section. To simplify the construction and analysis, we further assume $f$ has a known superpolynomial security $s(n)=n^{\omega(1)}$. (Haitner et al. [25] do not make this assumption, and we will also not need it in our final construction based on an arbitrary one-way function.)

Observe that the statistical hiding property of the NOVY commitment scheme based on one-way permutation $f$ relies only on the fact that $f$ is a permutation because we require that $f\left(U_{n}\right)$ be uniform. Now if $f$ just a regular function, then $f\left(U_{n}\right)$ might no longer be uniform, but instead all we can say is that $f\left(U_{n}\right)$ is a flat distribution with support $\operatorname{Supp}\left(f\left(U_{n}\right)\right)$ of size $2^{t}$. We will use pairwise-independent hash functions, a notion to be discussed next, to obtain an almost-uniform distribution from $f\left(U_{n}\right)$.

4.1. Hashing and randomness extraction. The entropy of a random variable $X$ is

$$
\mathrm{H}(X)=\underset{x \leftarrow X}{\mathrm{E}}\left[\frac{1}{\log \operatorname{Pr}[X=x]}\right]
$$

where here and throughout all logarithms are of base 2. This notion of entropy corresponds to Shannon entropy or information entropy in the information theory literature. Intuitively, $\mathrm{H}(X)$ measures the amount of randomness in $X$ on average (in 
bits). For a worst-case measure of randomness, the min-entropy of $X$ is most often used and is defined as

$$
\mathrm{H}_{\infty}(X)=\min _{x}\left[\frac{1}{\log \operatorname{Pr}[X=x]}\right] .
$$

In general, $\mathrm{H}_{\infty}(X) \leq \mathrm{H}(X)$, but when $X$ is flat (i.e., uniform on its support), then $\mathrm{H}(X)=\mathrm{H}_{\infty}(X)=\log |\operatorname{Supp}(X)|$.

A family of hash functions $\mathcal{H}=\left\{h:\{0,1\}^{n} \rightarrow\{0,1\}^{m}\right\}$ is pairwise-independent (a.k.a. strongly 2-universal) if for any two $x \neq x^{\prime} \in\{0,1\}^{n}$ and any two $y, y^{\prime} \in\{0,1\}^{m}$ when we randomly choose $h \leftarrow \mathcal{H}$, we have $\operatorname{Pr}\left[h(x)=y\right.$ and $\left.h\left(x^{\prime}\right)=y^{\prime}\right]=2^{-2 m}$.

An example of a pairwise-independent family of hash functions for $n \geq m$ is the family $\mathcal{H}=\left\{h_{a, b}:\{0,1\}^{n} \rightarrow\{0,1\}^{m}\right\}$, where $h_{a, b}(x)=\left.(a \cdot x+b)\right|_{m}$, arithmetic is done in the field $\mathrm{GF}\left(2^{n}\right)$, and $\left.\right|_{m}$ denotes taking the first $m$ bits. We define $\ell(n, m)$ to be the number of bits required to describe an element of the hash function family $\mathcal{H}$. In our example, it takes $2 n$ bits to describe each hash function $h_{a, b}$ since both $a$ and $b$ are elements of $\mathrm{GF}\left(2^{n}\right)$; hence, a family of pairwise-independent hash functions $\mathcal{H}$ mapping $n$-bit strings to $m$-bit strings exists with $\ell(n, m)=2 n$. We will use the following property of pairwise-independent hash functions to obtain an almost-uniform random variable from a random variable with sufficient min-entropy.

Lemma 4.1 (Leftover Hash Lemma [37, 5]). Let random variable $H$ denote a uniformly random hash function from a family of pairwise-independent hash functions $\mathcal{H}$ mapping $n$-bit strings to $m$-bit strings, and let $X$ be a random variable taking values in $\{0,1\}^{n}$. For any $\varepsilon>0$, if $\mathrm{H}_{\infty}(X) \geq m+2 \log (1 / \varepsilon)$, and $H$ is independent from $X$, then the random variable $(H, H(X))$ is $\varepsilon$-close in statistical distance to uniform.

4.2. The commitment scheme. Let us return to our regular one-way function $f:\{0,1\}^{n} \rightarrow\{0,1\}^{n}$ with known preimage size $2^{n-t}$ and known security $s(n)=n^{\omega(1)}$. Consider a family of pairwise-independent hash functions $\mathcal{H}=\left\{h:\{0,1\}^{n}\right.$ $\left.\rightarrow\{0,1\}^{t-\Delta}\right\}$, where $t=\mathrm{H}\left(f\left(U_{n}\right)\right)$ and $\Delta=\frac{1}{2} \log s(n)$. Let random variable $H$ represent a random hash function selected from $\mathcal{H}$. By the Leftover Hash Lemma, Lemma 4.1, random variable $Z=\left(H, H\left(f\left(U_{n}\right)\right)\right)$ is $(1 / s(n))^{\Omega(1)}$-close to uniform, which statistically gives indistinguishability from uniform because $s(n)=n^{\omega(1)}$. So if we designate $z=(h, h(f(x)))$ as the sender's private input to the interactive hashing protocol (Protocol 3.3), even an all-powerful receiver will not get more than a negligible advantage to guess which one of the outputs is $z$. This hints at the following commitment scheme.

Protocol 4.2. Commitment scheme $(S, R)$ is based on a regular one-way function $f:\{0,1\}^{n} \rightarrow\{0,1\}^{n}$ with known preimage size $2^{n-t}$ and known security $s(n)=n^{\omega(1)}$.

Commit stage.

1. Let $\mathcal{H}=\left\{h:\{0,1\}^{n} \rightarrow\{0,1\}^{t-\Delta}\right\}$, where $t=\mathrm{H}\left(f\left(U_{n}\right)\right)$ and $\Delta=$ $\frac{1}{2} \log s(n)$. S selects a uniform $x \leftarrow\{0,1\}^{n}$ and a hash function $h \leftarrow \mathcal{H}$ and computes $y=f(x)$ and $z=(h, h(y))$.

2. $S$ and $R$ engage in interactive hashing (Protocol 3.3) with $S$ acting as $S_{\mathrm{IH}}, R$ acting as $R_{\mathrm{IH}}$, parameters $k=1$ and $q=|z|$, and $S_{\mathrm{IH}}$ having private input $z$. Their common output is a circuit $C:\{0,1\} \rightarrow\{0,1\}^{q}$, and the sender receives a bit $d \in\{0,1\}$ such that $C(d)=z$.

3. To commit to the bit $b, S$ sends $c=d \oplus b$ to $R$. The commitment of $b$ is represented as the pair $(C, c)$. 
Reveal stage. To decommit, $S$ sends bits $b$ and $d$, string $x$, and hash function $h$ to $R$. $R$ verifies the decommitment by checking if $c=d \oplus b$ and $C(d)=(h, h(f(x)))$.

As we have argued previously, the sender's private input $z$ is statistically close to uniform, and hence by the hiding property of interactive hashing, this implies that the commitment scheme is statistically hiding. As for the binding property, the one-wayness of $f$ intuitively guarantees that the set $T$ of $w$ 's for which a sender $S^{*}$ can compute an element of $f^{-1}(w)$ is of density at most $1 / s(n)$ in the range of $f$; that is, the size of $T$ is at most $2^{\mathrm{H}\left(f\left(U_{n}\right)\right)-\log s(n)}$. Thus, for any fixed $h$, the fraction of $z=(h, h(w))$ such that $w \in T$ is at most $2^{\mathrm{H}\left(f\left(U_{n}\right)\right)-\log s(n)} / 2^{t-\Delta}=s(n)^{-1 / 2}=$ neg $(n)$. By the binding property of interactive hashing stated in Lemma 3.7, the probability that $S^{*}$ can force both $C(0), C(1) \in T$ is negligible, and hence, the scheme is computationally binding. The complete argument to prove the binding property is actually more subtle because the set $T$ is not actually fixed in advance, and so we need to employ the stronger binding property given in Definition 3.1. We omit the full proof, because this is merely a warm-up example to motivate our actual construction and proof in subsequent sections.

5. 1-out-of-2-binding commitments from unknown-regular one-way functions. Our next hurdle is to remove to the constraint on knowing (i.e., being able to efficiently compute) the preimage size. For this setting, let us consider a regular one-way function $f:\{0,1\}^{n} \rightarrow\{0,1\}^{n}$ with preimage size $2^{n-t}$, for an unknown ${ }^{6}$ value of $t \in\{1,2, \ldots, n\}$, but with known security $s(n)=n^{\omega(1)} .^{7}$ Constructing statistically hiding commitments even in this setting was still an open problem prior to our work.

Let us examine why we need to know the correct value of $t$ in the previous scheme of Protocol 4.2. If the value of $t$ is too high, that is, $t \gg \mathrm{H}\left(f\left(U_{n}\right)\right)$, then the scheme is no longer hiding (but would be binding). This is because the Leftover Hash Lemma, Lemma 4.1, no longer applies, since in this case the min-entropy $\mathrm{H}\left(f\left(U_{n}\right)\right)$ is too small relative to $t$. On the other hand, if the value of $t$ is too low, that is, $t \ll \mathrm{H}\left(f\left(U_{n}\right)\right)$, then the scheme is no longer binding (but would be hiding). To see this, at least intuitively, observe that when $t$ is very small, we are hashing $f\left(U_{n}\right)$ to a very small set $\{0,1\}^{t-\Delta}$; in other words, $h$ collapses too many elements in $f\left(U_{n}\right)$. As a consequence, inverting $h\left(f\left(U_{n}\right)\right)$ could be easy (even though inverting $f\left(U_{n}\right)$ is hard), and this allows us to break the binding property of our scheme.

All hope, however, is not lost. We can still use Protocol 4.2, trying all values of $t \in\{1,2, \ldots, n\}$, to do our first phase commitments. And to overcome the difficulty of ensuring both hiding and binding, we will introduce a second phase that will be binding when $t \lesssim \mathrm{H}\left(f\left(U_{n}\right)\right)$ and hiding when $t \gtrsim \mathrm{H}\left(f\left(U_{n}\right)\right)$; this is obtained by the sender using a hash of the preimage $x$ as an input to another execution of interactive hashing. This means that for the right value of $t=\mathrm{H}\left(f\left(U_{n}\right)\right)$, both phases will be hiding, but for any value of $t$, at least one phase is binding. What we are describing here is a 2-phase commitment scheme with a 1-out-of-2 binding property, notions that we formally define in the next section.

5.1. 2-phase commitment schemes. As mentioned previously, we will work with 2-phase commitment schemes, an alternate variant of commitments introduced

\footnotetext{
${ }^{6}$ What we mean by unknown is that we are not able to compute the preimage size efficiently.

${ }^{7}$ Like in section 4 , we consider only length-preserving functions, that is, $|f(x)|=|x|$ for all $x \in\{0,1\}^{*}$, to avoid introducing new parameters. Our construction can nevertheless be easily generalized to regular one-way functions that are not length-preserving.
} 
by Nguyen and Vadhan [28]. These are commitment schemes with two sequential and related stages such that in each stage, the sender commits to and reveals a value.

Definition 5.1. A 2-phase commitment scheme $(S, R)$, with security parameter $n$ and message lengths $\left(k_{1}(n), k_{2}(n)\right)$, consists of four polynomial-time interactive protocols: the first commitment stage $\left(S_{c}^{1}, R_{c}^{1}\right)$, the first reveal stage $\left(S_{r}^{1}, R_{r}^{1}\right)$, the second commitment stage $\left(S_{c}^{2}, R_{c}^{2}\right)$, and the second reveal stage $\left(S_{r}^{2}, R_{r}^{2}\right)$. (We also write $S=\left(S^{1}, S^{2}\right)$ and $R=\left(R^{1}, R^{2}\right)$ to denote the first and second phases of $S$ and $R$, respectively.) For us, both reveal phases will always be noninteractive, consisting of a single message from the sender to the receiver.

1. In the first commitment stage, $S_{c}^{1}$ receives a private input $\sigma^{(1)} \in\{0,1\}^{k_{1}}$ and coin tosses $r_{S}$. At the end of the interaction, both $S_{c}^{1}$ and $R_{c}^{1}$ output a commitment $c^{(1)}$. (Without loss of generality, we can assume that $c^{(1)}$ is the transcript of the first commitment stage.)

2. In the first (noninteractive) reveal stage, both $S_{r}^{1}$ and $R_{r}^{1}$ receive as common input the commitment $c^{(1)}$, and $S_{r}^{1}$ receives as private input its previous coin tosses $r_{S} . S_{r}^{1}$ sends $R_{r}^{1}$ a pair $\left(\sigma^{(1)}, \gamma^{(1)}\right)$ with $\gamma^{(1)}$ interpreted as a decommitment for $\sigma^{(1)} \in\{0,1\}^{k_{1}}$. $R_{r}^{1}$ accepts or rejects based on $c^{(1)}, \sigma^{(1)}$, and $\gamma^{(1)}$. After that, both $S_{r}^{1}$ and $R_{r}^{1}$ output a string $\tau$. (Without loss of generality, we can assume that $\tau$ is the transcript of the first commitment stage and the first reveal stage and includes $R_{r}^{1}$ 's decision to accept or reject.)

3. In the second commitment stage, both $S_{c}^{2}$ and $R_{c}^{2}$ receive as common input the string $\tau$, and $S_{c}^{2}$ receives a private input $\sigma^{(2)} \in\{0,1\}^{k_{2}}$ and its previous coin tosses $r_{S}$. At the end of the interaction, both $S_{c}^{2}$ and $R_{c}^{2}$ output a commitment $c^{(2)}$. (Without loss of generality, we can assume that $c^{(2)}$ is the concatenation of $\tau$ and the transcript of the second commitment stage.)

4. In the second (noninteractive) reveal stage, both $S_{r}^{2}$ and $R_{r}^{2}$ receive as common input the commitment $c^{(2)}$, and $S_{r}^{2}$ receives as private input its previous coin tosses $r_{S}$. $S_{r}^{2}$ sends $R_{r}^{2}$ a pair $\left(\sigma^{(2)}, \gamma^{(2)}\right)$ with $\gamma^{(2)}$ interpreted as a decommitment for $\sigma^{(2)} \in\{0,1\}^{k_{2}} . R_{r}^{2}$ accepts or rejects based on $c^{(2)}, \sigma^{(2)}$, and $\gamma^{(2)}$.

- We insist that scheme $(S, R)$ have perfect completeness. That is to say, if both sender $S$ and receiver $R$ follow their prescribed strategies, then $R$ will always accept in both phases (with probability 1 ).

- The sender and receiver's algorithms, denoted by $S=\left(S^{1}, S^{2}\right)=\left(\left(S_{c}^{1}, S_{r}^{1}\right)\right.$, $\left.\left(S_{c}^{2}, S_{r}^{2}\right)\right)$ and $R=\left(R^{1}, R^{2}\right)=\left(\left(R_{c}^{1}, R_{r}^{1}\right),\left(R_{c}^{2}, R_{r}^{2}\right)\right)$, respectively, are computable in polynomial time.

- Scheme $(S, R)$ is public coin if all messages sent by $R$ to $S$ are independent random coins.

Remark 5.2 (2-phase commitment schemes). We make several remarks regarding Definition 5.1.

1. We generally consider schemes that have the same message length for both phases. When this is the case, namely, $k=k_{1}=k_{2}$, we say our 2-phase commitment scheme has message length $k$. It is only in section 7 that we will use the feature of different message lengths.

2. Instead of providing sender $S$ with decommitment values as private outputs of the commitment phases, we simply provide it with the same coin tosses $r_{S}$ throughout (so it can recompute any private state from the transcripts of the previous phases). The receiver $R$, however, operates using independent coin tosses in each phase and does not need to keep a private state. 
Hiding for 2-phase commitment schemes. As for standard commitment schemes, we define the security of the sender in terms of a hiding property. Stated informally, the hiding property for a 2-phase commitment scheme says that both commitment phases are hiding. Note that since the phases are run sequentially, the hiding property for the second commitment stage is required to hold even given the receiver's view of the first stage.

DEFINITION 5.3. 2-phase commitment scheme $(S, R)$, with security parameter $n$ and message lengths $\left(k_{1}(n), k_{2}(n)\right)$, is statistically hiding if, for all adversarial receivers $R^{*}$, the following hold:

1. The views of $R^{*}$ when interacting with the sender in the first phase on any two messages are statistically indistinguishable. Namely, for all $\sigma^{(1)}, \widetilde{\sigma}^{(1)} \in$ $\{0,1\}^{k_{1}}$, the probability ensembles $\quad\left\{\operatorname{view}_{R^{*}}\left(S_{c}^{1}\left(\sigma^{(1)}\right), R^{*}\right)\left(1^{n}\right)\right\}_{n \in \mathbb{N}}$ and $\left\{\operatorname{view}_{R^{*}}\left(S_{c}^{1}\left(\widetilde{\sigma}^{(1)}\right), R^{*}\right)\left(1^{n}\right)\right\}_{n \in \mathbb{N}}$ are statistically indistinguishable.

2. The views of $R^{*}$ when interacting with the sender in the second phase are statistically indistinguishable no matter what the sender committed to in the first phase. Namely, for all $\sigma^{(1)} \in\{0,1\}^{k_{1}}$, and all $\sigma^{(2)}, \widetilde{\sigma}^{(2)} \in\{0,1\}^{k_{2}}$, the probability ensembles $\left\{\operatorname{view}_{R^{*}}\left(S_{c}^{2}\left(\sigma^{(2)}\right), R^{*}\right)\left(\mathrm{T}, 1^{n}\right)\right\}_{n \in \mathbb{N}}$ and $\left\{\operatorname{view}_{R^{*}}\left(S_{c}^{2}\left(\widetilde{\sigma}^{(2)}\right), R^{*}\right)\right.$ $\left.\left(\mathrm{T}, 1^{n}\right)\right\}_{n \in \mathbb{N}}$, where $\mathrm{T}=\operatorname{transcript}\left(S^{1}\left(\sigma^{(1)}\right), R^{*}\right)\left(1^{n}\right)$, are statistically indistinguishable.

We stress that the second condition of the above hiding definition (Definition 5.3) requires that the view of receiver in the second phase be indistinguishable for any two messages even given the transcript of the first phase, $\mathrm{T}=\operatorname{transcript}\left(S^{1}\left(\sigma^{(1)}\right), R^{*}\right)\left(1^{n}\right)$.

1-out-of-2 binding for 2-phase commitment schemes. The 1-out-of-2 binding property, informally stated, says that at least one of the two commitment phases is binding. In other words, for every PPT malicious sender $S^{*}$, at most one of the two phases is bad in that $S^{*}$ can decommit a given commitment to two different messages in that phase. We allow this bad phase to be determined dynamically by $S^{*}$. Moreover, we require that the second phase be statistically binding if the sender breaks the first phase. Our construction achieves this stronger property, and using it simplifies some of our proofs.

DEFINITION 5.4. 2-phase commitment scheme $(S, R)$, with security parameter $n$ and message lengths $\left(k_{1}(n), k_{2}(n)\right)$, is computationally 1-out-of-2 binding if there exists a "binding set" $\mathcal{B}$ of first phase transcripts such that for every function $\varepsilon(n)=$ $1 / \operatorname{poly}(n)$, the following hold:

1. Every PPT $S^{*}$ succeeds in the following game with probability at most $\varepsilon(n)$ for all sufficiently large $n$ :

(a) $S^{*}$ and $R_{c}^{1}$ interact and output a first-phase commitment $c^{(1)}$.

(b) $S^{*}$ outputs two full transcripts $\lambda=(\tau, \kappa)$ and $\widetilde{\lambda}=(\widetilde{\tau}, \widetilde{\kappa})$ of both phases with the following three properties:

- Transcripts $\lambda$ and $\widetilde{\lambda}$ both start with prefix $c^{(1)}$.

- Transcript $\lambda$ contains a successful opening of $c^{(1)}$ to the value $\sigma^{(1)} \in$ $\{0,1\}^{k_{1}}$ using a first-phase transcript $\tau$ not in $\mathcal{B}$, and $R_{r}^{1}$ and $R_{r}^{2}$ both accept in $\lambda$.

- Transcript $\tilde{\lambda}$ contains a successful opening of $c^{(1)}$ to the value $\widetilde{\sigma}^{(1)} \in$ $\{0,1\}^{k_{1}}$ using a first-phase transcript $\widetilde{\tau}$ not in $\mathcal{B}$, and $R_{r}^{1}$ and $R_{r}^{2}$ both accept in $\widetilde{\lambda}$.

(c) $S^{*}$ succeeds if all of the above conditions hold and $\sigma^{(1)} \neq \widetilde{\sigma}^{(1)}$.

2. For every (even computationally unbounded) sender $S^{*}$, the first-phase transcripts in $\mathcal{B}$ make the second phase statistically binding. In other words, for 
all $S^{*}$, all $\tau \in \mathcal{B}$, and all sufficiently large $n$, with probability at least $1-\varepsilon(n)$ over $c^{(2)}=\left(S^{*}, R_{c}^{2}\right)(\tau)$, there is at most one value $\sigma^{(2)} \in\{0,1\}^{k_{2}}$ such that $\exists \gamma^{(2)} R_{r}^{2}\left(c^{(2)}, \sigma^{(2)}, \gamma^{(2)}\right)=$ accept.

Some remarks on this definition:

1. Note that we require condition 1 to hold against PPT adversaries, but condition 2 must hold against all, even computationally unbounded, adversaries.

2. Condition 2 has an equivalent reformulation that is more similar to condition 1. Specifically, we can require for all $S^{*}$, all $\tau \in \mathcal{B}$, and all sufficiently large $n, S^{*}$ succeeds in the following game with probability at most $\varepsilon(n): S^{*}$ and $R_{c}^{2}$ interact to get a second-phase commitment $c^{(2)}$. After the interaction $S^{*}$ outputs a pair of full transcripts $\lambda, \tilde{\lambda}$ and we say that $S^{*}$ succeeds if both $\lambda$ and $\tilde{\lambda}$ begin with prefix $c^{(2)}$ (which in turn contains $\tau$ as a prefix), $R_{r}^{2}$ accepts in both $\lambda$ and $\tilde{\lambda}$, and $\lambda$ and $\tilde{\lambda}$ contain decommitments to two distinct values $\sigma \neq \tilde{\sigma}$.

3. Conversely, an equivalent reformulation of condition 1 says that for every PPT $S^{*}$ and every $\varepsilon(n)=1 / \operatorname{poly}(n)$, the following holds for all sufficiently large $n$ : with probability at least $1-\varepsilon(n)$ over $c^{(1)} \stackrel{R}{\leftarrow}\left(S^{*}, R_{c}^{1}\right)$, there is at most one value $\sigma^{(1)} \in\{0,1\}^{k_{1}}$ for which there is at least an $\varepsilon(n)$ probability (conditioned on $\left.c^{(1)}\right)$ that $S^{*}$ subsequently outputs a full accepting transcript $\lambda=(\tau, \kappa)$ with $\tau=\left(c^{(1)}, \sigma^{(1)}, \gamma^{(1)}\right) \notin \mathcal{B}$. Thus, we think of $c^{(1)}$ as a commitment to this unique value of $\sigma^{(1)}$.

4. Note that for $S^{*}$ to break condition 1 (or its equivalent formulation above), it must produce a full transcript that makes both $R_{r}^{1}$ and $R_{r}^{2}$ accept. Thus, the decommitment for the first phase should not really be considered "complete" until a corresponding second-phase transcript is produced. The reason we do not have the honest sender provide such a "full decommitment" during the first-phase reveal is that this would compromise the hiding property of the second phase.

5. If a 2-phase commitment is both statistically hiding and computationally 1-out-of-2 binding, then the transcripts $\tau$ generated in the first-phase interaction between the honest sender $\left(S^{1}, R^{1}\right)$ will lie outside $\mathcal{B}$ with all but negligible probability. Indeed, the statistical hiding property of the second phase implies that the second phase cannot be statistically binding (except with negligible probability), and thus by condition 2 the first-phase transcript $\tau$ must be outside of $\mathcal{B}$.

5.2. Our 2-phase commitment scheme. We now describe our 2-phase commitment scheme for general functions $f:\{0,1\}^{n} \rightarrow\{0,1\}^{n}$, not necessarily regular nor one-way - as we shall later see, it is the regularity condition that gives the hiding property and the one-wayness of the function that gives the binding property of our scheme. Let $\mathcal{H}=\left\{h:\{0,1\}^{n} \rightarrow\{0,1\}^{m}\right\}$ be a family of pairwise-independent hash functions. As shown in section 4.1, we have a family whose description of each element takes $\ell(n, m)=2 n$ bits. It will be convenient to make $\ell(n, m)+m=q(n)$ for some fixed polynomial $q(n)$, so that for every $y \in\{0,1\}^{n},|h, h(y)|=q(n)$. This can be done by padding random bits to the description of $h$.

In addition, it will be convenient to work with protocols where the sender has no input $\sigma^{(j)}$ to be committed to, but rather privately receives an output $d^{(j)}$ at the end of each phase $j \in\{1,2\}$ of the commitment. If we can ensure that $d^{(j)}$ is close to uniform given the receiver's view, such a protocol can be easily turned into a commitment scheme: the sender can commit to a $\sigma^{(j)}$ of its choice by sending 
$d^{(j)} \oplus \sigma^{(j)}$ at the end of the commit stage.

On the other hand, it will be convenient to have a special private input $x \in\{0,1\}^{n}$ that corresponds to a portion of sender's coin tosses. The reason for this is that later we will want to discuss properties of the protocol when $x$ is chosen randomly from a subset $\Gamma \subseteq\{0,1\}^{n}$ rather than uniformly from the entire space.

Protocol 5.5. 2-phase commitment scheme $(S, R)$ based on $f:\{0,1\}^{n} \rightarrow$ $\{0,1\}^{n}$.

Parameters: Integers $t \in\{1,2, \ldots, n\}, k_{1}=k_{2}=k \in\{1,2, \ldots, n\}, \Delta_{1} \in\{0,1, \ldots, t\}$, and $\Delta_{2} \in\{0,1, \ldots, n-t\}$.

Sender's (special) private input: String $x \in\{0,1\}^{n}$. (Again, this is not the value to which the sender is committing, but is rather part of its coins, which will be chosen uniformly at random by $S$ unless otherwise specified.)

First-phase commit:

1. $S_{c}^{1}$ sets $y=f(x)$.

2. Let $\mathcal{H}_{1}=\left\{h_{1}:\{0,1\}^{n} \rightarrow\{0,1\}^{t-\Delta_{1}}\right\}$ be a family of pairwise-independent hash functions. $S_{c}^{1}$ chooses a random hash $h_{1} \leftarrow \mathcal{H}_{1}$ and computes $z^{(1)}=\left(h_{1}, h_{1}(y)\right) \in\{0,1\}^{q}$.

3. $\left(S_{c}^{1}, R_{c}^{1}\right)$ run the interactive hashing protocol $\left(S_{\mathrm{IH}}\left(z^{(1)}\right), R_{\mathrm{IH}}\right)\left(1^{q}, 1^{k}\right)$, given by Protocol 3.3, with $S_{c}^{1}$ and $R_{c}^{1}$ acting as $S_{\mathrm{IH}}$ and $R_{\mathrm{IH}}$, respectively. Let circuit $C^{(1)}:\{0,1\}^{k} \rightarrow\{0,1\}^{q}$ be the common output and $d^{(1)} \in\{0,1\}^{k}$ (with $\left.C^{(1)}\left(d^{(1)}\right)=z^{(1)}\right)$ be $S_{\mathrm{IH}}$ 's private output in $\left(S_{\mathrm{IH}}\left(z^{(1)}\right)\right.$, $\left.R_{\mathrm{IH}}\right)\left(1^{q}, 1^{k}\right)$.

First-phase sender's private output: String $d^{(1)} \in\{0,1\}^{k}$.

First-phase reveal:

$S_{r}^{1}$ sends the tuple $\gamma^{(1)}=\left(d^{(1)}, y, h_{1}\right)$.

Receiver $R_{r}^{1}$ accepts if and only if $C^{(1)}\left(d^{(1)}\right)=\left(h_{1}, h_{1}(y)\right)$.

Second-phase commit:

Second-phase common input: First-phase transcript $\tau=\operatorname{transcript}\left(S^{1}(x), R^{1}\right)$, which in particular includes the string $y$.

1. Let $\mathcal{H}_{2}=\left\{h_{2}:\{0,1\}^{n} \rightarrow\{0,1\}^{n-t-\Delta_{2}}\right\}$ be a family of pairwise-independent hash functions. $S_{c}^{2}$ chooses a random hash $h_{2} \leftarrow \mathcal{H}_{2}$ and computes $z^{(2)}=\left(h_{2}, h_{2}(x)\right) \in\{0,1\}^{q}$.

2. $\left(S_{c}^{2}, R_{c}^{2}\right)$ run the interactive hashing protocol $\left(S_{\mathrm{IH}}(w), R_{\mathrm{IH}}\right)\left(1^{q}, 1^{k}\right)$, given by Protocol 3.3, with $S_{c}^{2}$ and $R_{c}^{2}$ acting as $S_{\mathrm{IH}}$ and $R_{\mathrm{IH}}$, respectively. Let circuit $C^{(2)}:\{0,1\}^{k} \rightarrow\{0,1\}^{q}$ be the common output and $d^{(2)} \in\{0,1\}^{k}$ be $S_{\mathrm{IH}}$ 's private output in $\left(S_{\mathrm{IH}}\left(z^{(2)}\right), R_{\mathrm{IH}}\right)\left(1^{q}, 1^{k}\right)$.

Second-phase sender's private output: String $d^{(2)} \in\{0,1\}^{k}$.

Second-phase reveal:

$S_{r}^{2}$ sends the tuple $\gamma^{(2)}=\left(d^{(2)}, x, h_{2}\right)$.

Receiver $R_{r}^{2}$ accepts if and only if $f(x)=y$ and $C^{(2)}\left(d^{(2)}\right)=\left(h_{2}, h_{2}(x)\right)$.

TheOREM 5.6. Suppose $f:\{0,1\}^{n} \rightarrow\{0,1\}^{n}$ is a regular one-way function with (known) security $s(n)=n^{\omega(1)}$. Then for any setting of parameters such that $t \in\left[\mathrm{H}\left(f\left(U_{n}\right)\right), \mathrm{H}\left(f\left(U_{n}\right)\right)+1\right], k=O(\log n)$, and $\Delta_{1}=\Delta_{2}=\frac{1}{4} \log s \leq \max \{t, n-t\}$, Protocol 5.5 is a 2-phase commitment scheme that is statistically hiding and computationally 1-out-of-2 binding. Moreover, the computational 1-out-of-2 binding property holds regardless of the setting of $t$ or the regularity of $f$. 
In order to apply the above theorem, it needs to be verified that if we set $t=$ $\left\lfloor\mathrm{H}\left(f\left(U_{n}\right)\right)\right\rfloor$, then $\frac{1}{4} \log s \leq \min \{t, n-t\}$. This can be easily made to hold by padding the input of $f$. Specifically, given a one-way function $f:\{0,1\}^{n} \rightarrow\{0,1\}^{n}$, the function $f^{\prime}:\{0,1\}^{3 n} \rightarrow\{0,1\}^{3 n}$ defined by $f^{\prime}(x, y, z)=(f(x), y)$ for $|x|=|y|=|z|$ is a regular one-way function with security $s \leq 2^{n}$ and output entropy $\mathrm{H}\left(f^{\prime}\left(U_{n^{\prime}}\right)\right) \in$ $[n, 2 n]$, so $\frac{1}{4} \log s \leq n / 4<\min \left\{t, n^{\prime}-t\right\}$, where $n^{\prime}=2 n$ is the input length of $f^{\prime}$.

Because we do not know how to efficiently compute the correct value of $t=$ $\mathrm{H}\left(f\left(U_{n}\right)\right)$, we are forced to try out all values of $t=1,2, \ldots, n$ to get a collection of commitment schemes, as stated in the next corollary. While having a collection of schemes instead of a single scheme may seem inconvenient, in section 7 we will show how to convert such a collection of 2-phase commitments into a single commitment scheme that is statistically hiding and computationally binding (in the standard sense of binding).

Corollary 5.7. Given a regular one-way function $f:\{0,1\}^{n} \rightarrow\{0,1\}^{n}$ with known security $s(n)=n^{\omega(1)}$, we can construct in time polynomial in $n$ a collection of public-coin 2-phase commitment schemes $\mathcal{C O M}=\left\{\mathrm{Com}_{1}, \ldots, \mathrm{Com}_{n}\right\}$, such that

- there exists an index $i \in\{1,2, \ldots, n\}$ such that scheme $\mathrm{Com}_{i}$ is statistically hiding, and

- for every index $i \in\{1,2, \ldots, n\}$, scheme $\mathrm{Com}_{i}$ is computationally 1-out-of-2 binding.

For notational convenience and generality, the above corollary and some of our subsequent results are stated in terms of finite functions $f:\{0,1\}^{n} \rightarrow\{0,1\}^{n}$ for a fixed value $n$ of the security parameter. When we say that the function $f$ is "given," this can be interpreted as being given the boolean circuit computing $f$ (or, more generally, given $f$ as an oracle), and "constructing" the commitment schemes $\mathrm{Com}_{i}$ can be interpreted as constructing the boolean circuits (or, more generally, circuits with oracle gates for evaluating $f$ ) that compute the next-message functions of the protocol.

We divide the proof of Theorem 5.6 into Lemmas 5.8 and 5.9, which establish the statistical hiding and computational 1-out-of-2 binding properties of Protocol 5.5, respectively.

Lemma 5.8. If $f:\{0,1\}^{n} \rightarrow\{0,1\}^{n}$ is a regular function, then Protocol 5.5, with any setting of parameters satisfying $t \in\left[\mathrm{H}\left(f\left(U_{n}\right)\right), \mathrm{H}\left(f\left(U_{n}\right)\right)+1\right], k<q(n), \Delta_{1}=$ $\Delta_{2}=\omega(\log n) \leq \min \{t, n-t\}$, is statistically hiding in the sense of Definition 5.3.

Proof. Since $t \leq \mathrm{H}\left(f\left(U_{n}\right)\right)+1$, the Leftover Hash Lemma (Lemma 4.1) tells us that random variable $Z^{(1)}=\left(H_{1}, H_{1}\left(f\left(U_{n}\right)\right)\right)$ is $2^{-\Omega\left(\Delta_{1}\right)}$-close to the uniform. Then by the hiding property of interactive hashing (Definition 3.1), the first commitment phase is $2^{-\Omega\left(\Delta_{1}\right)}$-hiding, which suffices because $\Delta_{1}=\omega(\log n)$.

Let $\tau$ be the transcript of the first phase and $y$ the string sent in the first reveal phase. Let random variable $X$ represent selecting at random a string from the set $f^{-1}(y)$. Since $X$ is a flat source with entropy $n-\mathrm{H}\left(f\left(U_{n}\right)\right) \geq n-t$, and $h_{2}$ maps to strings of length $n-t-\Delta_{2}$, we apply the Leftover Hash Lemma once more to conclude that random variable $Z^{(2)}=\left(H_{2}, H_{2}(X)\right)$ is $2^{-\Omega\left(\Delta_{2}\right)}$-close to the uniform, even given $\tau$. By the hiding property of interactive hashing, the second commitment phase is $2^{-\Omega\left(\Delta_{2}\right)}$-hiding, which in turn is statistically hiding since $\Delta_{2}=\omega(\log n)$.

Lemma 5.9. If $f:\{0,1\}^{n} \rightarrow\{0,1\}^{n}$ is an $s(n)$-secure one-way function (not necessarily regular), then for any value of $t \in\{1,2, \ldots, n\}$, Protocol 5.5, with any 
setting of parameters such that $k=O(\log n), \Delta_{1}=\Delta_{2} \leq \min \left\{\frac{1}{4} \log (s(n)), t, n-t\right\}$, is computationally 1-out-of-2 binding in the sense of Definition 5.4.

Intuitively, the proof of Lemma 5.9 will proceed as follows. We will show that with high probability the first commit phase binds the (cheating) sender to a unique "heavy" string $y$, i.e., a string whose probability mass under $f\left(U_{n}\right)$ is at least roughly $2^{-t}$. During the first reveal phase, the sender can choose to reveal either this heavy string or a "light" string. In the former case, the first phase is computationally binding, and in the latter, the second phase is statistically binding.

Formally, we define the binding set $\mathcal{B}$ as follows:

For every $t \in\{1,2, \ldots, n\}$, define the set of light strings to be $L_{t}=$ $\left\{y \in\{0,1\}^{n}: \operatorname{Pr}\left[f\left(U_{n}\right)=y\right] \leq 2^{-t-\Delta_{3}}\right\}$ for a parameter $\Delta_{3}$ that we will set at the end of the proof. Define the binding set $\mathcal{B}$ to be the set of transcripts where the sender reveals $y \in L_{t}$.

Now, we break the proof of Lemma 5.9 into Claims 5.10 and 5.11 below, which establish the binding property for the first and second phases, respectively.

Claim 5.10. For the binding set $\mathcal{B}$ defined above, if there exists a PPT $S^{*}$ that succeeds with probability $\varepsilon=\varepsilon(n)$ in the game in condition 1 of Definition 5.4, then there exists a PPT B that can invert $f$ with success probability at least

$$
\varepsilon^{O(1)} \cdot 1 / \operatorname{poly}(n) \cdot 2^{-\left(k+\Delta_{1}+\Delta_{3}\right)} .
$$

Proof. We define a relation $W$ as follows:

$$
W=\left\{\left(z^{(1)}, x\right): \exists h_{1} \text { such that both } z^{(1)}=\left(h_{1}, h_{1}(f(x))\right) \text { and } f(x) \notin L_{t}\right\} .
$$

Suppose we have a PPT $S^{*}$ that succeeds with probability greater than $\varepsilon$ in the game described in condition 1 of Definition 5.4. In particular, this means that $S^{*}$ after interacting with $R_{\mathrm{IH}}$ will, with probability greater than $\varepsilon$, produce pairs $\left(d_{0}^{(1)}, x_{0}\right)$ and $\left(d_{1}^{(1)}, x_{1}\right)$ such that $d_{0}^{(1)} \neq d_{1}^{(1)},\left(C^{(1)}\left(d_{0}\right), x_{0}\right) \in W$, and $\left(C^{(1)}\left(d_{1}\right), x_{1}\right) \in W$. By the binding property of interactive hashing (condition 3 of Definition 3.1), there exists a PPT $A$ such that

$$
z^{(1)} \operatorname{Pr}_{\{0,1\}^{q}}\left[A\left(z^{(1)}\right) \in W_{z^{(1)}}\right]>2^{-k} \cdot\left(\frac{\varepsilon}{q}\right)^{O(1)},
$$

where the above probability is taken over the coin tosses of $A$ and $v \leftarrow\{0,1\}^{q}$.

Consider an algorithm $B$ that on input $y$ picks a random hash function $h_{1} \leftarrow \mathcal{H}_{1}$ and outputs $A\left(h_{1}, h_{1}(y)\right)$. We let $\eta=h_{1}(y)$ and compute the probability that $B$ 
inverts $f$ as follows:

$$
\begin{aligned}
& \operatorname{Pr}\left[B\left(f\left(U_{n}\right)\right) \in f^{-1}\left(f\left(U_{n}\right)\right)\right] \\
= & \underset{h_{1} \leftarrow \mathcal{H}_{1}}{\mathrm{E}}\left[\sum_{x} \operatorname{Pr}\left[A\left(h_{1}, h_{1}\left(f\left(U_{n}\right)\right)\right)=x \wedge f(x)=f\left(U_{n}\right)\right]\right] \\
= & \underset{h_{1} \leftarrow \mathcal{H}_{1}}{\mathrm{E}}\left[\sum_{\eta, x \text { s.t. } \eta=h_{1}(f(x))} \operatorname{Pr}\left[f\left(U_{n}\right)=f(x)\right] \cdot \operatorname{Pr}\left[A\left(h_{1}, \eta\right)=x\right]\right] \\
\geq & \underset{h_{1} \leftarrow \mathcal{H}_{1}}{\mathrm{E}}\left[\sum_{\eta, x \text { s.t. } x \in W_{\left(h_{1}, \eta\right)}} \operatorname{Pr}\left[f\left(U_{n}\right)=f(x)\right] \cdot \operatorname{Pr}\left[A\left(h_{1}, \eta\right)=x\right]\right] \\
\geq & \underset{h_{1} \leftarrow \mathcal{H}_{1}}{\mathrm{E}}\left[\sum_{\eta, x \text { s.t. } x \in W_{\left(h_{1}, \eta\right)}} 2^{-t-\Delta_{3}} \cdot \operatorname{Pr}\left[A\left(h_{1}, \eta\right)=x\right]\right]\left(x \in W_{\left(h_{1}, \eta\right)} \Rightarrow f(x) \notin L_{t}\right) \\
= & 2^{-t-\Delta_{3}} \cdot 2^{t-\Delta_{1}} \cdot\left(_{\left(h_{1}, \eta\right) \leftarrow \mathcal{H}_{1} \times\{0,1\}^{t-\Delta_{1}}}\left[A\left(h_{1}, \eta\right) \in W_{\left(h_{1}, \eta\right)}\right]\right. \\
> & 2^{-\left(\Delta_{1}+\Delta_{3}\right)} \cdot 2^{-k} \cdot\left(\frac{\varepsilon}{q}\right)^{O(1)}(\text { by }(1)) \\
= & \varepsilon^{O(1)} \cdot 1 / \operatorname{poly}(n) \cdot 2^{-\left(k+\Delta_{1}+\Delta_{3}\right)}(\operatorname{since} q=\operatorname{poly}(n)) .
\end{aligned}
$$

Claim 5.11. For the binding set $\mathcal{B}$ defined above, condition 2 of Definition 5.4 is satisfied with $\varepsilon=\operatorname{poly}(n) \cdot 2^{-\Omega\left(\Delta_{3}-\Delta_{2}\right)} \cdot 2^{k / 2}$.

Proof. Let $y \in L_{t}$ be the string sent in the first reveal phase. This means that $\operatorname{Pr}\left[f\left(U_{n}\right)=y\right] \leq 2^{-t-\Delta_{3}}$, or equivalently $\left|f^{-1}(y)\right| \leq 2^{n-t-\Delta_{3}}$. Define set $T=$ $\left\{\left(h_{2}, h_{2}(x)\right): h_{2} \in \mathcal{H}_{2}, x \in f^{-1}(y)\right\}$, and let $\mu(T)$ denote the density of the subset $T$. Since $h_{2}$ maps $\{0,1\}^{n}$ to $\{0,1\}^{n-t-\Delta_{2}}$, we have

$$
\mu(T) \leq \frac{\left|f^{-1}(y)\right|}{2^{n-t-\Delta_{2}}} \leq \frac{2^{n-t-\Delta_{3}}}{2^{n-t-\Delta_{2}}}=2^{\Delta_{2}-\Delta_{3}} .
$$

Applying Lemma 3.7, we have

$\operatorname{Pr}\left[\left(z_{0}^{(2)}, z_{1}^{(2)}\right)=\operatorname{output}\left(S^{*}, R_{\mathrm{IH}}\right)\right.$ satisfies $\left.z_{0}^{(2)}, z_{1}^{(2)} \in T\right]<\operatorname{poly}(q) \cdot 2^{-\Omega\left(\Delta_{3}-\Delta_{2}\right)} \cdot 2^{k / 2}$,

which then concludes our proof since $q$ is a fixed polynomial in $n$.

Proof of Lemma 5.9. Set $\Delta_{3}=\frac{1}{2} \log s(n)$, and we are given that $k=O(\log n)$ and $\Delta_{1}=\Delta_{2} \leq \frac{1}{4} \log (s(n))$. With this setting, Claim 5.11 shows that condition 2 in Definition 5.4 is satisfied with $\varepsilon(n)=\operatorname{poly}(n) \cdot 2^{-\Omega(\log s(n))}=\operatorname{neg}(n)$, since $s(n)=n^{\omega(1)}$. Condition 1 of Definition 5.4 is also satisfied with negligible probability $\varepsilon(n)$ because otherwise $f$ can be inverted with probability

$$
\begin{aligned}
\varepsilon^{O(1)} \cdot 1 / \operatorname{poly}(n) \cdot 2^{-\left(k+\Delta_{1}+\Delta_{3}\right)} & \geq \varepsilon^{O(1)} \cdot 1 / \operatorname{poly}(n) \cdot 2^{-(O(\log n)+(3 / 4) \cdot(\log s(n)))} \\
& =\varepsilon^{O(1)} \cdot 1 / \operatorname{poly}(n) \cdot s(n)^{-3 / 4},
\end{aligned}
$$

which is greater than $1 / s(n)$ if $\varepsilon$ is nonnegligible.

6. 1-out-of-2-binding commitments from any one-way function. Our next hurdle is to remove the regularity assumption. It turns out that this is the 
most technically challenging step. (We also need to remove the need for known security, but this will follow as a byproduct of our approach to removing the regularity assumption.) Similar to our construction from regular one-way functions (with unknown preimage size) in section 5, our construction based on any one-way function yields a collection of 2-phase commitments, as stated below.

THeOREM 6.1. Given a one-way function $f:\{0,1\}^{n} \rightarrow\{0,1\}^{n}$, we can construct in time polynomial in $n$ a collection of $m=\operatorname{poly}(n)$ public-coin 2-phase commitment schemes $\mathcal{C O M}=\left\{\mathrm{Com}_{1}, \ldots, \mathrm{Com}_{m}\right\}$ with message lengths $\left(k_{1}, k_{2}\right)=(n, n)$, such that

- there exists an index $i \in\{1,2, \ldots, m\}$ such that scheme $\mathrm{Com}_{i}$ is statistically hiding, and

- for every index $i \in\{1,2, \ldots, m\}$, scheme $\operatorname{Com}_{i}$ is computationally 1-out-of-2 binding.

Note that this theorem provides 2-phase commitment schemes for long messages, specifically those with length equal to the input length $n$ of the one-way function $f$. Essentially the same proof can provide schemes with message length $k(n)$ for any desired polynomial $k$. Alternatively, we can apply the above theorem to the function $f^{\prime}\left(x_{1}, \ldots, x_{k}\right)=\left(f\left(x_{1}\right), \ldots, f\left(x_{k}\right)\right)$, which has input length $n^{\prime}=k \cdot n$ and is one-way if $f$ is.

A collection of 2-phase commitment schemes as above turns out to suffice for obtaining statistical zero-knowledge arguments for all of NP (see $[28,1])$. Hence, Theorem 6.1 suffices to establish Theorem 1.2, which states that statistical zero-knowledge arguments for all of NP can be based on any one-way function. However, in section 7, we will show how to transform the above collection of 2-phase commitment schemes into a single commitment scheme that is statistically hiding and computationally binding (in the standard sense of binding). This proves Theorem 1.1, the main theorem of this paper, and gives a more modular proof of Theorem 1.2 (simply by plugging the commitments into [9]).

We prove Theorem 6.1 in sections $6.1-6.3$.

6.1. Overview. We now present an overview of how we generalize our construction for regular one-way functions with unknown preimage size (Protocol 5.5) to arbitrary one-way functions. As shown in Lemma 5.9, this protocol already achieves 1 -out-of-2 binding when $f$ is any one-way function (for every value of $t$ ). Thus the challenge is the hiding property. (Another issue is that Protocol 5.5 requires a oneway function with known security. It turns out that our method for handling the hiding property also eliminates the need to know the security.)

As discussed in section 5, for regular one-way functions with unknown preimage size, Protocol 5.5 has a hiding first phase when the parameter $t$ satisfies $t \lesssim \mathrm{H}\left(f\left(U_{n}\right)\right)$ and has a hiding second phase when $t$ satisfies $t \gtrsim \mathrm{H}\left(f\left(U_{n}\right)\right)$. Intuitively, when $f$ is not regular, we should replace the fixed value $\mathrm{H}\left(f\left(U_{n}\right)\right)$ with the dynamic value $\mathrm{H}_{f}(y) \stackrel{\text { def }}{=} \log \left(1 / \operatorname{Pr}\left[f\left(U_{n}\right)=y\right]\right)$, where $y=f(x)$ is the value chosen by the sender in the preprocessing step, because $\mathrm{H}_{f}(y)$ can be viewed as measuring the amount of entropy in $y$. The approximable preimage-size one-way functions studied by Haitner et al. [25] come equipped with an algorithm that estimates $\mathrm{H}_{f}(y)$, but for general one-way functions, this quantity may be infeasible to compute.

A weakly hiding scheme (details in section 6.2). One natural approach is to have the sender choose $t$ at random and hope that it is close to $\mathrm{H}_{f}(y)$. When we choose $t$ too small, only the first phase will be hiding, and when we choose $t$ too large, only the second phase will be hiding. But we have a nonnegligible probability of $\delta=1 / n$ that $t \approx \mathrm{H}_{f}(y)$, and thus both phases will be hiding. Thus we have a 1- 
out-of-2-binding commitment scheme satisfying a weak hiding property, where with probability $\delta=1 / n$, both phases are hiding, and it is always the case that at least one phase is hiding. Actually, in order to simplify our analysis, we will include $t$ as a parameter to the protocol. Then there exists a choice of $t$ such that the protocol is weakly hiding in the sense above, and for all choices of $t$ the protocol is 1-out-of-2 binding. At the end, we will enumerate over all values of $t$, resulting in a collection of commitment schemes as claimed in Theorem 6.1, albeit with a weak hiding property.

Unfortunately, we do not know how to directly construct statistically hiding commitments from weakly hiding 1-out-of-2-binding commitments. Thus instead, much of the effort in this paper is devoted to amplifying the weak hiding property, where $\delta=1 / n$, into a strong hiding property, where $\delta=1-\operatorname{neg}(n)$, while maintaining the 1-out-of-2 binding property. ${ }^{8}$

Amplifying the hiding property (details in section 6.3). We do not amplify the hiding probability from $\delta=1 / n$ to $\delta=1-\operatorname{neg}(n)$ in one shot, but instead perform a sequence of $\log n$ iterations, each one of which increases $\delta$ by roughly a factor of 2 . This results in $\delta=\Omega(1)$, and then we are able to get $\delta=1-\operatorname{neg}(n)$ in just one more amplification step.

How do we double $\delta$ ? A natural idea is to consider several executions of the previous weakly hiding scheme. Specifically, if we take $m=O(1)$ executions, the probability that at least one of the executions has both phases hiding is roughly $m \cdot \delta$. Moreover, each of the remaining $m-1$ executions have either the first phase hiding or the second phase hiding. Thus, for some value of $\beta$, there are $\beta+1$ first phases that are hiding and $m-\beta$ second phases that are hiding. It turns out that we can choose $\beta$ so that this exact $(\beta+1, m-\beta)$ breakdown given that one execution has both phases hiding occurs with probability $\Omega(1 / \sqrt{m})$. Thus we are in the situation described with probability $m \cdot \delta \cdot \Omega(1 / \sqrt{m})=\Omega(\sqrt{m} \cdot \delta)>2 \delta$ for a large enough constant $m$.

Now our aim is to combine the outcomes of the weakly hiding schemes in such a way that when the above-described situation occurs, which happens with probability at least $2 \delta$, both phases are hiding. Notice that the secret values $\sigma_{1}, \ldots, \sigma_{m} \in\{0,1\}^{k}$ to which the sender commits in the first commit phases have entropy (even minentropy) at least $(\beta+1) \cdot k$ conditioned on the receiver's view (because $(\beta+1)$ of them are hiding), and similarly the sender's secrets in the second commit phases have entropy at least $(m-\beta) \cdot k$ conditioned on the receiver's view. Let us compare this to the situation with binding. Since each execution is 1-out-of-2 binding, a cheating polynomial-time sender can break the binding property for either at most $\beta$ of the first phases or at most $m-\beta-1$ of the second phases. Thus the number of possible values to which the sender can open in each case is at most $2^{m} \cdot 2^{k \cdot \beta}$ in the first phase or at most $2^{k \cdot(m-\beta-1)}$, where the $2^{m}$ factor in the first bound comes from the sender's ability to choose which subset of executions to break (and it is this factor that limits us to taking $m$ to be a constant). We can view these as strong forms of entropy upper bounds $m+k \beta$ and $k \cdot(m-\beta-1)$. In at least one phase, there will be an entropy gap of at least $k-m$.

How can we exploit these entropy gaps? It turns out that interactive hashing, again, is a useful tool. Specifically, in the first phase we have the sender choose a ran-

\footnotetext{
${ }^{8}$ One may wonder why we do not now apply the compiler of section 7 , which converts strongly hiding 2-phase commitments into standard commitments. Even if that compiler could be made to work for weakly hiding 2-phase commitments (producing a weakly hiding standard commitment), another obstacle is that the compiler requires a 2-phase commitment with polynomial message length, whereas our weakly hiding commitment has only a logarithmic message length (similarly to Theorem 5.6). It turns out that our amplification procedure also increases the message length as needed.
} 
dom pairwise-independent hash function $h_{1}$ mapping to approximately $(\beta+1) \cdot k$ bits and use $\left(h_{1}, h_{1}\left(\sigma_{1}, \ldots, \sigma_{m}\right)\right)$ as the input to the interactive hashing protocol, and analogously for the second phase. By the Leftover Hash Lemma, this pairwise-independent hashing results in an almost-uniform distribution, so the interactive hashing hiding property applies. As for the binding property, the bound on the number of the sender's choices gets translated to saying that the sender's input (in the first phase) comes from a set $\Gamma$ of density $2^{-(k-m)}$, so the interactive hashing binding property applies. The analysis for the second phase is similar. Formalizing these ideas, we get a new 1-out-of-2-binding commitment scheme in which both phases are hiding with probability at least $2 \delta$.

When we try to iterate this amplification step $O(\log n)$ times, we run into a new difficulty. Specifically, the above sketch hides the fact that we pay entropy losses of $\omega(\log n)$ in both the hiding and binding analyses. The entropy loss of $\omega(\log n)$ in the hiding property comes from the Leftover Hash Lemma in order to ensure that $\left(h_{1}, h_{1}\left(\sigma_{1}, \ldots, \sigma_{m}\right)\right)$ has negligible statistical distance from uniform. The entropy loss of $\omega(\log n)$ in the binding property comes from needing the $\mu(\Gamma) \cdot 2^{k}$ factor to be negligible when applying Lemma 3.7. This forces us to go, in one step of amplification, from a commitment scheme for secrets of length $k$ to a scheme for secrets of length $k-m-\omega(\log n)$. As in Lemma 5.9, we can take the initial secret to be of length $k=\Theta(\log s(n))=\omega(\log (n))$ if the one-way function has known security $s(n)=n^{\omega(1)}$. But to tolerate $\log n \operatorname{losses}$ of $\omega(\log n)$, we would need $s(n)=n^{\omega(\log n)}$ (i.e., at least quasi-polynomial security).

To get around this difficulty, in the amplification, we work with more relaxed, average-case measures of entropy for both the hiding and binding analyses. Specifically, for hiding, we keep track of the expected collision probability of the sender's secret, conditioned on the receiver's view. (Actually, we use the expected square root of the collision probability.) For binding, we work with the expected number of values to which the sender can open. In both cases, we require only that these expectations to be within a constant factor of the ideal values, which are $2^{-k}$ and 1 , respectively. With these measures, it turns out that we need only lose $O(m)=O(1)$ bits in the entropy gap with each amplification step. Moreover, once we amplify $\delta$ to a constant, we can afford to take the number of executions $m$ to equal the security parameter $n$ and get an $\Omega(n)$-bit entropy gap in the final amplification step. This allows us to achieve exponentially strong statistical hiding even when we do not know the security and start with secret length of $k=O(\log n)$.

The hiding analysis of the above construction works only for certain values of $t$ in the weakly hiding scheme, and for certain values of the $\beta$ 's in the amplification steps. We try out all possible values of $t$ and $\beta$ 's, thus obtaining a collection of $\operatorname{poly}(n)$ schemes, at least one of which is strongly hiding and all of which are 1-out-of2 binding. Notice that the number of possible choices of $t$ and the $\beta$ 's is polynomial in $n$ since $t \in\{1,2, \ldots, n\}$, the $\beta$ 's in the each step except for the last are in the range $\{0,1, \ldots, m-1\}$ for some constant $m$, and the last $\beta$ is in the range $\{0,1, \ldots, n\}$.

6.2. Weakly hiding and 1-out-of-2-binding commitments. As discussed in section 5, for the case of regular one-way functions with unknown preimage size, Protocol 5.5 has a hiding first phase when the parameter $t$ satisfies $t \lesssim \mathrm{H}\left(f\left(U_{n}\right)\right)$ and has a hiding second phase when $t$ satisfies $t \gtrsim \mathrm{H}\left(f\left(U_{n}\right)\right)$. When $f$ is not regular, then there will be one value of $t \in\{1,2, \ldots, n\}$ such that $\mathrm{H}_{f}(y) \approx t$ with probability $\gtrsim 1 / n$ over $y \stackrel{\text { R }}{\leftarrow} f\left(U_{n}\right)$, where $\mathrm{H}_{f}(y) \stackrel{\text { def }}{=} \log \left(1 / \operatorname{Pr}\left[f\left(U_{n}\right)=y\right]\right)$ measures the entropy in the specific output $y$ ). This is the case because there are only $n$ possible choices for the 
value of $t$.

With this observation in mind, our 2-phase commitment scheme from general one-way functions will be the same as the scheme in Protocol 5.5, with setting of parameters $t=t_{0}, k=O(\log n)$ and $\Delta_{1}=\Delta_{2}=2 \log n$, for some $t_{0} \in\{1,2, \ldots, n\}$. In other words, the same scheme - with slightly different setting of parameters - used in the case of regular one-way functions is also applicable to general one-way functions.

This commitment scheme (using general one-way functions), as we will show, is computationally 1-out-of-2 binding, but only statistically hiding in both phases with probability at least $1 / n$ (hence, called weakly hiding). In order to obtain a tighter analysis when we amplify this scheme, we depart from the standard measures of hiding and binding used in section 5. Instead, we measure the statistical hiding property of our 2-phase commitments using the expected square root of the collision probability of the sender's secret, denoted as $\mathrm{CP}^{1 / 2}$, and defined in section 6.2.1. We measure the binding property by analyzing the expected number of values to which an adversarial sender can open.

Later, in section 6.3 , we show how to boost the statistical hiding probability to $1-2^{-\Omega(n)}$ while maintaining the computational 1-out-of-2 binding property.

\subsubsection{Properties of collision probability.}

DeFINITION 6.2. For any random variable $A$, we define its collision probability as the probability that two independent samples from $A$ are equal. In other words,

$$
\mathrm{CP}(A) \stackrel{\text { def }}{=} \sum_{a \in \operatorname{Supp}(A)}(\operatorname{Pr}[A=a])^{2}=\underset{a \leftarrow A}{\mathrm{E}}[\operatorname{Pr}[A=a]] .
$$

Measuring the collision probability of a random variable is equivalent to measuring its Renyi entropy of order 2 , defined as

$$
\mathrm{H}_{2}(A)=\log \frac{1}{\mathrm{E}_{a \leftarrow A}[\operatorname{Pr}[A=a]]}=\log \frac{1}{\mathrm{CP}(A)} .
$$

Definition 6.3. For any random variable $A$, we denote the square root of its collision probability as

$$
\mathrm{CP}^{1 / 2}(A) \stackrel{\text { def }}{=} \sqrt{\mathrm{CP}(A)} .
$$

For any two (possibly correlated) random variables $A$ and $B$, we define the expected square root of $A$ 's collision probability given $B$ as

$$
\mathrm{CP}^{1 / 2}(A \mid B) \stackrel{\text { def }}{=} \underset{b \leftarrow B}{\mathrm{E}}\left[\mathrm{CP}^{1 / 2}\left(\left.A\right|_{B=b}\right)\right] .
$$

We think of $\mathrm{CP}^{1 / 2}(A \mid B) \leq \sqrt{2^{k}}$ as saying that $A$ has conditional Renyi entropy of at least $k$ given $B$. We use the expected square root of the collision probability (as our measure of hiding) instead of just expected collision probability in order to ensure that conditioning on a random variable $Z$ can only decrease the conditional Renyi entropy by at most $\log (|\operatorname{Supp}(Z)|)$ bits. (See Lemma 6.7 below for details.)

The following lemmas show that $\mathrm{CP}^{1 / 2}$ behaves nicely as an entropy measure. Proofs are in the appendix.

Lemma 6.4. For independent pairs of random variables $\left(X_{1}, Y_{1}\right), \ldots,\left(X_{m}, Y_{m}\right)$,

$$
\mathrm{CP}^{1 / 2}\left(\left(X_{1}, \ldots, X_{m}\right) \mid\left(Y_{1}, \ldots, Y_{m}\right)\right)=\prod_{i=1}^{m} \mathrm{CP}^{1 / 2}\left(X_{i} \mid Y_{i}\right) .
$$


Note that $X_{i}$ and $Y_{i}$ can be correlated; it is required only that the pair $\left(X_{i}, Y_{i}\right)$ be independent from the other tuples.

In terms of conditional Renyi entropy, Lemma 6.4 states that the entropy is additive for independent random variables. We will actually need a generalization of Lemma 6.4 to deal with somewhat dependent random variables, as stated in the next lemma.

Lemma 6.5. Suppose random variables $\left(X_{1}, Y_{1}\right), \ldots,\left(X_{m}, Y_{m}\right)$ satisfy the following conditions for some values of $\alpha_{1}, \ldots, \alpha_{m} \in \mathbb{R}^{+}$and all $i=1,2, \ldots, m$ :

1. For every $\left(y_{1}, \ldots, y_{i-1}\right) \in \operatorname{Supp}\left(Y_{1}, Y_{2}, \ldots, Y_{i-1}\right)$,

$$
\mathrm{CP}^{1 / 2}\left(\left.X_{i}\right|_{Y_{1}=y_{1}, \ldots, Y_{i-1}=y_{i-1}}\left|Y_{i}\right|_{Y_{1}=y_{1}, \ldots, Y_{i-1}=y_{i-1}}\right) \leq \alpha_{i} .
$$

2. For every $\left(y_{1}, \ldots, y_{i}\right) \in \operatorname{Supp}\left(Y_{1}, Y_{2}, \ldots, Y_{i}\right)$, the $i+1$ random variables $X_{1}, X_{2}, \ldots, X_{i}$, and $Y_{i+1}$ are independent after conditioning on $Y_{1}=y_{1}, \ldots, Y_{i}$ $=y_{i}$.

Then,

$$
\mathrm{CP}^{1 / 2}\left(\left(X_{1}, \ldots, X_{m}\right) \mid\left(Y_{1}, \ldots, Y_{m}\right)\right) \leq \prod_{i=1}^{m} \alpha_{i} .
$$

The next lemma shows that pairwise-independent randomness extraction $(h, h(x))$ preserves the $\mathrm{CP}^{1 / 2}$ measure.

Lemma 6.6 (Randomness Extraction Lemma). Let $(X, Y)$ be any (possibly correlated) pair of random variables, and let random variable $H$ denote a random hash function from a family of pairwise-independent hash functions $\mathcal{H}$ with range $\{0,1\}^{\alpha}$. Suppose the hash functions from $\mathcal{H}$ are represented by $(q-\alpha)$-bit strings and $\mathrm{CP}^{1 / 2}(X \mid Y) \leq \sqrt{2^{-(\alpha+3)}}$. If $H$ is independent from $(X, Y)$, then

$$
\mathrm{CP}^{1 / 2}((H, H(X)) \mid Y) \leq \sqrt{2^{-(q-1)}} .
$$

In other words, if $X$ has at least $\alpha+3$ bits of conditional Renyi entropy given $Y$, then we can extract $\alpha$ bits from $X$ that have conditional Renyi entropy at least $\alpha-1$. Notice that this entropy loss is only 4 bits, as compared to $2 \log (1 / \varepsilon)$ if we require that the output be $\varepsilon$-close to uniform as in the Leftover Hash Lemma (Lemma 4.1). This constant loss of conditional Renyi entropy allows us to do a tighter hiding analysis in section 6.3.1.

LEMma 6.7. For any triple of (possibly correlated) random variables $X, Y$, and $Z$,

$$
\mathrm{CP}^{1 / 2}(X \mid Y) \leq \mathrm{CP}^{1 / 2}(X \mid(Y, Z)) \leq \sqrt{|\operatorname{Supp}(Z)|} \cdot \mathrm{CP}^{1 / 2}(X \mid Y)
$$

This says that conditioning on random variable $Z$ can only decrease the conditional Renyi entropy, but does so by at most $\log (|\operatorname{Supp}(Z)|)$ bits. For Shannon entropy, a stronger statement can be proven, namely, that conditioning on $Z$ reduces entropy by at most $\mathrm{H}(Z)$ bits: $\mathrm{H}(X \mid Z) \geq \mathrm{H}(X)-\mathrm{H}(Z)$ (omitting the random variable $Y$ for simplicity). This follows from the chain rule $\mathrm{H}(X, Z)=\mathrm{H}(Z)+\mathrm{H}(X \mid Z)$, noting that $\mathrm{H}(X, Z) \geq \mathrm{H}(X)$. However, the chain rule does not hold for conditional Renyi 
entropy. ${ }^{9}$

The final lemma is a stronger variant of the previous Leftover Hash Lemma (Lemma 4.1), with its hypothesis stated in terms of collision probability.

Lemma 6.8 (Leftover Hash Lemma, strengthened $[37,5]$ ). Let random variable $H$ denote a random hash function from a family of pairwise-independent hash functions $\mathcal{H}$ with range $\{0,1\}^{\alpha}$. For any $\varepsilon>0$, if $\mathrm{CP}(X) \leq \varepsilon^{2} \cdot 2^{-\alpha}$ and $H$ is independent from $X$, then random variable $(H, H(X))$ is $\varepsilon$-close in statistical distance to uniform.

6.2.2. Average-case hiding and binding properties of interactive hashing. We now analyze the interactive hashing protocol, namely, Protocol 3.3, in terms of average-case measures. For hiding, we use the $\mathrm{CP}^{1 / 2}$ measure introduced in the previous section. For the binding property, we present an average-case variant of Lemma 3.7, where we look at the expected number of outputs that lie in any set $\Gamma$, rather than bound the probability that there is more than one output in $\Gamma$.

LEMMA 6.9 (hiding of interactive hashing in $\mathrm{CP}^{1 / 2}$ measure). Let $\left(S_{\mathrm{IH}}, R_{\mathrm{IH}}\right)$ be the interactive hashing protocol in Protocol 3.3. If the sender $S_{\mathrm{IH}}$ 's input comes from a random variable $Y$ over $\{0,1\}^{q}$ and $W$ is any (possibly correlated) random variable (representing the receiver's a priori information about $Y$ ), then for any receiver $R^{*}$,

$$
\mathrm{CP}^{1 / 2}(Z \mid(W, V)) \leq \sqrt{2^{q-k}} \cdot \mathrm{CP}^{1 / 2}(Y \mid W),
$$

where $Z=$ output $_{S_{\mathrm{IH}}}\left(S_{\mathrm{IH}}(Y), R^{*}\right)\left(1^{q}, 1^{k}\right)$ and $V=\operatorname{view}_{R^{*}}\left(S_{\mathrm{IH}}(Y), R^{*}\right)\left(1^{q}, 1^{k}\right)$.

Proof. Without loss of generality, we may assume that $R^{*}$ is deterministic. (The randomized case then follows by taking expectation over $R^{*}$ 's coins.) Now that $R^{*}$ is deterministic, the hash functions sent $h_{0}, \ldots, h_{q-k-1}$ by $R^{*}$ are fully determined by $S_{\mathrm{IH}}$ 's responses $c_{0}, \ldots, c_{q-k-1} \in\{0,1\}$ (refer to Protocol 3.3). Hence, the number of possible different views of $R^{*}$ is bounded by $2^{q-k}$. This implies that $|\operatorname{Supp}(V)| \leq 2^{q-k}$, where $V=\operatorname{view}_{R^{*}}\left(S_{\mathrm{IH}}(Y), R^{*}\right)\left(1^{q}, 1^{k}\right)$. By Lemma 6.7,

$$
\mathrm{CP}^{1 / 2}(Y \mid(W, V)) \leq \sqrt{|\operatorname{Supp}(V)|} \cdot \mathrm{CP}^{1 / 2}(Y \mid W) \leq \sqrt{2^{q-k}} \cdot \mathrm{CP}^{1 / 2}(Y \mid W) .
$$

Observe that given any particular instantiation of $W=w$ and $V=v$, the distribution of output $\left._{S_{\mathrm{IH}}}\left(S_{\mathrm{IH}}(Y), R^{*}\right)\left(1^{q}, 1^{k}\right)\right|_{W=w, V=v}$ has the same collision probability as $\left.Y\right|_{W=w, V=v}$ (indeed, they are in bijective correspondence). Hence, $\mathrm{CP}^{1 / 2}(Z \mid(W, V))=$ $\mathrm{CP}^{1 / 2}(Y \mid(W, V)) \leq \sqrt{2^{q-k}} \cdot \mathrm{CP}^{1 / 2}(Y \mid W)$.

LEMMA 6.10 (binding of interactive hashing in expected measure). Let $\left(S_{\mathrm{IH}}, R_{\mathrm{IH}}\right)$ be the interactive hashing protocol in Protocol 3.3. For any fixed subset $T \subseteq\{0,1\}^{q}$, and for any sender $S^{*}$, setting $C=\operatorname{output}\left(\left(S^{*}, R_{\mathrm{HH}}\right)\left(1^{q}, 1^{k}\right)\right)$, we have

$$
\mathrm{E}[|\{z: C(z) \in T\}|]<\max \left\{24,2^{k+1} \cdot \mu(T)\right\} \leq 24+2^{k+1} \cdot \mu(T),
$$

where the above expectation is taken over the coins of $S^{*}$ and $R_{\mathrm{IH}}$.

This lemma and its proof are inspired by the work of Goldreich, Goldwasser, and Linial [38], who studied a protocol similar to interactive hashing for a different purpose (namely, random selection protocols).

\footnotetext{
${ }^{9}$ In terms of collision probability, a chain rule would say $\mathrm{CP}^{1 / 2}(X, Z)=\mathrm{CP}^{1 / 2}(Z) \cdot \mathrm{CP}^{1 / 2}(X \mid Z)$. This is not true in general, as can be observed by noting that $\left(\mathrm{CP}^{1 / 2}(X, Z) / \mathrm{CP}^{1 / 2}(Z)\right)^{2}=$ $\mathrm{CP}(X, Z) / \mathrm{CP}(Z)$ equals the expectation $\mathrm{CP}\left(\left.X\right|_{Z=z}\right)$ over $z$ chosen according to the distribution $\hat{Z}$, where $\operatorname{Pr}[\hat{Z}=z]=\operatorname{Pr}[Z=z]^{2} / \operatorname{CP}(Z)$, while $\mathrm{CP}^{1 / 2}(X \mid Z)$ (and any natural definition of conditional Renyi entropy) is defined by taking an expectation over $Z$. By choosing a random variable $Z$ where $\hat{Z}$ is very different from $Z$, and choosing appropriate conditional distributions $\left.X\right|_{Z=z}$, examples can be constructed where $\mathrm{CP}^{1 / 2}(X, Z)$ is both much larger and much smaller than $\mathrm{CP}^{1 / 2}(Z) \cdot \mathrm{CP}^{1 / 2}(X \mid Z)$.
} 
Proof. Without loss of generality, we may assume that $S^{*}$ is deterministic. (Else, we can fix its coins to maximize the expectation.) Note that for iteration $j=0, \ldots, q-$ $k-1, R_{\mathrm{IH}}$ will send a random $h_{j}$, partitioning the set of possible outputs into two sets $\left\{y: h_{j}(y)=0\right\}$ and $\left\{y: h_{j}(y)=1\right\}$, and $S^{*}$ chooses a side of the partition by sending a bit $c_{j}$. For all $j \geq 0$, let $U_{j}=\left\{y \in\{0,1\}^{q}: h_{i}(y)=c_{i} \forall i<j\right\}$ denote the set of compatible elements of the universe at iteration $j$, and let $T_{j}=U_{j} \cap T$ denote the set of compatible elements of $T$. We write $\mu\left(T_{j}\right)=\left|T_{j}\right| /\left|U_{j}\right|=\left|T_{j}\right| \cdot 2^{-(q-j)}$ and denote the density of $T_{j}$ within $U_{j}$, and $\left.\mu_{j}=\mathrm{E}_{[}[\mu]\left(T_{j}\right)\right]$, where the expectation is taken over random choices of $h_{0}, \ldots, h_{j-1}$. For convenience of notation, we denote the hash function $h_{i}$ 's range as $\{ \pm 1\}$ instead of $\{0,1\}$.

Consider a particular set $T_{j}$ and a particular hash function $h_{j}$. Observe that for every $y \neq y^{\prime} \in T_{j}, \operatorname{Pr}_{h_{j}}\left[h_{j}(y)=h_{j}\left(y^{\prime}\right)\right] \leq 1 / 2$. Hence,

$$
\underset{h_{j}}{\mathrm{E}}\left[h_{j}(y) h_{j}\left(y^{\prime}\right)\right] \leq 0 \quad \forall y \neq y^{\prime} \in T_{j} .
$$

Observe that the set $T_{j+1}=\left\{y \in T_{j}: h_{j}(y)=c_{j}\right\}$, and thus

$$
\left|T_{j_{1}}\right| \leq \max _{c \in\{ \pm 1\}}\left|\left\{y \in T_{j}: h_{j}(y)=c\right\}\right|=\frac{\left|T_{j}\right|+\left|\sum_{y \in T_{j}} h_{j}(y)\right|}{2} .
$$

Therefore,

$$
\begin{aligned}
\underset{h_{j}}{\mathrm{E}}\left[\mu\left(T_{j+1}\right)\right] & =\mu\left(T_{j}\right)+2^{-(q-j)} \cdot \underset{h_{j}}{\mathrm{E}}\left[\left|\sum_{y \in T_{j}} h_{j}(y)\right|\right] \\
& \left.\leq \mu\left(T_{j}\right)+2^{-(q-j)} \cdot \sqrt{\underset{h_{j}}{\mathrm{E}}\left[\left(\sum_{y \in T_{j}} h_{j}(y)\right)^{2}\right]} \quad \text { (Cauchy-Schwarz/Jensen }\right) \\
& =\mu\left(T_{j}\right)+2^{-(q-j)} \cdot \sqrt{\left|T_{j}\right|+\sum_{y \neq y^{\prime}} \underset{h_{j}}{\mathrm{E}}\left[h_{j}(y) h_{j}\left(y^{\prime}\right)\right]} \\
& \left.\leq \mu\left(T_{j}\right)+2^{-(q-j)} \cdot \sqrt{\left|T_{j}\right|} \quad \text { (by } 2\right) \\
& =\mu\left(T_{j}\right)+\sqrt{2^{-(q-j)} \cdot \mu\left(T_{j}\right)} .
\end{aligned}
$$

Consequently,

$$
\begin{aligned}
\mu_{j+1} & =\underset{h_{0}, \ldots, h_{j}}{\mathrm{E}}\left[\mu\left(T_{j+1}\right)\right] \\
& =\underset{h_{0}, \ldots, h_{j-1}}{\mathrm{E}}\left[\mathrm{E}\left[\mu\left(T_{j+1}\right)\right]\right] \\
& \leq \underset{h_{0}, \ldots, h_{j-1}}{\mathrm{E}}\left[\mu\left(T_{j}\right)+\sqrt{2^{-(q-j)} \cdot \mu\left(T_{j}\right)}\right] \\
& \leq \underset{h_{0}, \ldots, h_{j-1}}{\mathrm{E}}\left[\mu\left(T_{j}\right)\right]+\sqrt{2^{-(q-j)} \cdot \underset{h_{0}, \ldots, h_{j-1}\left[\mu\left(T_{j}\right)\right]}{\mathrm{E}} \quad \text { (Cauchy-Schwarz/Jensen) }} \\
& =\mu_{j}+\sqrt{2^{-(q-j)} \cdot \mu_{j}} .
\end{aligned}
$$


Assume that the $\mu_{j}$ 's are monotonically increasing (otherwise, we can make it so). This gives us

$$
\begin{array}{rlrl}
\mu_{q-k} & \leq \mu_{0}+\sum_{j=0}^{q-k-1} \sqrt{2^{-(q-j)} \cdot \mu_{j}} & \\
& \leq \mu_{0}+\sqrt{\mu_{q-k}} \cdot \sum_{j=0}^{q-k-1} \sqrt{2^{-(q-j)}} & & \left(\mu_{j} \text { 's are monotonically increasing }\right) \\
& <\mu_{0}+\sqrt{\mu_{q-k}} \cdot \sqrt{6 / 2^{k}} & \\
& \leq \mu_{0}+\frac{\mu_{q-k}}{2} & & \left(\text { if } \mu_{q-k} \geq 24 \cdot 2^{-k}\right)
\end{array}
$$

giving us $\mu_{q-k}<2 \mu_{0}=2 \mu(T)$ if $\mu_{q-k} \geq 24 \cdot 2^{-k}$. This means that $\mu_{q-k}$ is either less than $24 \cdot 2^{-k}$ or less than $2 \mu(T)$. Therefore, we can conclude that

$$
\begin{aligned}
\mathrm{E}\left[|\{z: C(z) \in T\}|: C=\operatorname{output}\left(\left(S^{*}, R_{\mathrm{IH}}\right)\left(1^{q}, 1^{k}\right)\right)\right] & =\mu_{q-k} \cdot 2^{k} \\
& <\max \left\{24,2^{k+1} \cdot \mu(T)\right\} .
\end{aligned}
$$

6.2.3. Protocol 5.5 is hiding in $\mathbf{C P}^{1 / 2}$ measure. We are now ready to analyze the hiding property of Protocol 5.5 in terms of the $\mathrm{CP}^{1 / 2}$ measure. To do so, we say what it means for a scheme to be $\delta$-hiding in $\mathrm{CP}^{1 / 2}$ measure in Definition 6.11 below. But before going into that definition, we first establish some notation that is used throughout this part of the section.

With the sender's input being $x$, we let random variable view $R^{*}\left(S_{c}^{1}(x), R^{*}\right)$ denote the view of receiver $R^{*}$ in the first commit phase, random variable output ${ }_{S}\left(S_{c}^{1}(x), R^{*}\right)$ denote the sender's private output in the first phase, and random variable transcript $\left(S^{1}(x), R^{*}\right)$ denote the first (commit and reveal) phase transcript.

Using similar notation, with the transcript being $\tau$ and sender's input being $x$, we let random variable view $R^{*}\left(S_{c}^{2}(x), R^{*}\right)(\tau)$ denote the view of receiver $R^{*}$ in the second commit phase, random variable output ${ }_{S}\left(S_{c}^{2}(x), R^{*}\right)(\tau)$ denote the sender's private output in the second phase, and random variable transcript $\left(S^{2}(x), R^{*}\right)(\tau)$ denote the second (commit and reveal) phase transcript. We write $\Gamma_{1}$ in view $R^{*}\left(S_{c}^{1}\left(\Gamma_{1}\right), R^{*}\right)$ and similarly for others - to mean that the sender's private input is chosen uniformly from a set $\Gamma_{1}$.

Definition 6.11. For a parameter $\delta \in[0,1], 2$-phase commitment scheme $(S, R)$ is said to be $\delta$-hiding in $\mathrm{CP}^{1 / 2}$ measure if there exist two sets $\Gamma_{1}, \Gamma_{2} \subseteq\{0,1\}^{n}$ such that the following three properties hold.

(H.1) $\Gamma_{1} \cup \Gamma_{2}=\{0,1\}^{n}$ and $\mu\left(\Gamma_{1} \cap \Gamma_{2}\right) \geq \delta$.

(H.2) When the sender's private input $x$ is chosen uniformly from $\Gamma_{1}$, the sender's private output in the first phase has low collision probability given the receiver's view. Formally, for any adversarial receiver $R^{*}$,

$$
\mathrm{CP}^{1 / 2}(A \mid V) \leq \sqrt{2^{-(k-1)}}
$$

for $\left.(A, V)=\operatorname{output}_{S}\left(S_{c}^{1}\left(\Gamma_{1}\right), R^{*}\right), \operatorname{view}_{R^{*}}\left(S_{c}^{1}\left(\Gamma_{1}\right), R^{*}\right)\right)$.

(H.3) When the sender's private input $x$ is chosen uniformly from $\Gamma_{2}$, the sender's private output in the second phase has low collision probability given the receiver's view. Formally, for every adversarial receiver $R^{*}$ and every $\tau \in$ 
$\operatorname{Supp}(\mathrm{T})$, where $\mathrm{T}=\operatorname{transcript}\left(S^{1}\left(\Gamma_{2}\right), R^{*}\right)$, we have

$$
\mathrm{CP}^{1 / 2}\left(B_{\tau} \mid W_{\tau}\right) \leq \sqrt{2^{-(k-1)}}
$$

for $\left(B_{\tau}, W_{\tau}\right)=$ output $\left._{S}\left(S_{c}^{2}\left(\Gamma_{2}\right), R^{*}\right), \operatorname{view}_{R^{*}}\left(S_{c}^{2}\left(\Gamma_{2}\right), R^{*}\right)\right)\left.\right|_{\mathrm{T}=\tau}$.

Remark 6.12. Being $\delta$-hiding in $\mathrm{CP}^{1 / 2}$ measure in Definition 6.11 roughly means that the scheme is always hiding in at least one phase, and hiding in both phases occurs with probability $\delta$.

Lemma 6.13 (Protocol 5.5 is $(1 / n)$-hiding in $\mathrm{CP}^{1 / 2}$ measure). Let $f:\{0,1\}^{n} \rightarrow$ $\{0,1\}^{n}$ be any function (not necessarily one-way) such that $\left|f^{-1}(y)\right| \in\left[2 n^{2}, 2^{n} / n^{2}\right]$ for every $y \in\{0,1\}^{n}$. There exists an integer $t_{0} \in\{\lceil 2 \log n\rceil, 2, \ldots, n-\lceil 2 \log n\rceil\}$ such that Protocol 5.5, with setting of parameters $t=t_{0}, \Delta_{1}=\Delta_{2}=\lceil 2 \log n\rceil$, and any $k \in\{1, \ldots, q\}$, is $(1 / n)$-hiding in $\mathrm{CP}^{1 / 2}$ measure.

Proof. Without loss of generality, we may assume that $R^{*}$ is deterministic since we can fix the coins of $R^{*}$ that maximize the above collision probabilities. We prove that $(S, R)$ satisfies the above three properties of Definition 6.11 as follows.

Property (H.1). Define $p(y)=\operatorname{Pr}\left[f\left(U_{n}\right)=y\right]$, and for $t \in\{\lceil 2 \log n\rceil, 2, \ldots, n-$ $\lceil 2 \log n\rceil\}$, let $A_{t}=\left\{y \in\{0,1\}^{n}: 2^{-t} \leq p(y)<2^{-t+1}\right\}$. Since $\cup_{t} A_{t}=f\left(\{0,1\}^{n}\right)$, there exists an index $t_{0}$ such that $\operatorname{Pr}\left[f\left(U_{n}\right) \in A_{t_{0}}\right] \geq 1 / n$. Define sets $\Gamma_{1}$ and $\Gamma_{2}$ as follows:

$$
\begin{aligned}
& \Gamma_{1}=\left\{x: p(f(x))<2^{-t_{0}+1}\right\}, \\
& \Gamma_{2}=\left\{x: p(f(x)) \geq 2^{-t_{0}}\right\} .
\end{aligned}
$$

By the definition of $\Gamma_{1}$ and $\Gamma_{2}$, we have that $\mu\left(\Gamma_{1} \cap \Gamma_{2}\right)=\operatorname{Pr}\left[f\left(U_{n}\right) \in A_{t_{0}}\right] \geq 1 / n$, and also $\Gamma_{1} \cup \Gamma_{2}=\{0,1\}^{n}$.

Property (H.2). In the case when the sender's private input $x \in \Gamma_{1}$, we bound the collision probability of the first phase secret as follows:

$$
\begin{aligned}
\mathrm{CP}\left(f\left(\Gamma_{1}\right)\right) & =\sum_{y \in f\left(\Gamma_{1}\right)}\left(\frac{p(y)}{\mu\left(\Gamma_{1}\right)}\right)^{2} \\
& \leq\left(\max _{y \in f\left(\Gamma_{1}\right)} p(y)\right) \cdot\left(\sum_{y \in f\left(\Gamma_{1}\right)} p(y)\right) \cdot \frac{1}{\mu\left(\Gamma_{1}\right)^{2}} \\
& <2^{-t_{0}+1} \cdot \mu\left(\Gamma_{1}\right) \cdot \mu\left(\Gamma_{1}\right)^{-2} \\
& \left.\leq 2^{-\left(t_{0}-\log n-1\right)} \quad \quad \quad \text { (since } \mu\left(\Gamma_{1}\right) \geq 1 / n\right) .
\end{aligned}
$$

Observe that $\mathrm{CP}\left(f\left(\Gamma_{1}\right)\right) \leq 2^{-\left(t_{0}-\log n-1\right)} \leq 2^{-\left(t_{0}-\Delta_{1}+3\right)}$. Therefore, we can apply the Randomness Extraction Lemma, Lemma 6.6, to get $\mathrm{CP}^{1 / 2}\left(Z^{(1)}\right) \leq \sqrt{2^{-(q-1)}}$, where $Z^{(1)}=\left(H_{1}, H_{1}\left(f\left(\Gamma_{1}\right)\right)\right)$ and $H_{1}$ is an independent random hash from $\mathcal{H}_{1}$.

Next, let $A=$ output $_{S}\left(S_{c}^{1}\left(\Gamma_{1}\right), R^{*}\right)$ denote the private output of the sender $S$ in the first phase of Protocol 5.5, which in turn is equal to the output of $S_{\mathrm{IH}}$ in the interactive hashing protocol, so equivalently $A=$ output $_{S_{\mathrm{IH}}}\left(S_{\mathrm{IH}}\left(Z^{(1)}\right), R^{*}\right)$. Similarly, let $V=$ view $_{R^{*}}\left(S_{c}^{1}\left(\Gamma_{1}\right), R^{*}\right)$ denote the view of the adversarial receiver $R^{*}$ in the first phase, which in turn is equal to the view of $R^{*}$ in the interactive hashing protocol, so equivalently $V=\operatorname{view}_{R^{*}}\left(S_{\mathrm{IH}}\left(Z^{(1)}\right), R^{*}\right)$.

The final step is to use the hiding property of interactive hashing given by Lemma 6.9 to bound the collision probability of $A$ (the private output of the sender 
$S$ ) given $V$ (the view of the adversarial receiver $R^{*}$ ) as follows:

$$
\mathrm{CP}^{1 / 2}(A \mid V) \leq \sqrt{2^{q-k}} \cdot \sqrt{\mathrm{CP}\left(Z^{(1)}\right)} \leq \sqrt{2^{q-k}} \cdot \sqrt{2^{-(q-1)}}=\sqrt{2^{-(k-1)}} .
$$

Property (H.3). In the case when the sender's private input $x \in \Gamma_{2}$, we analyze the collision probability of the second phase secret as follows. First we observe that for any $x, x^{\prime} \in\{0,1\}^{n}$ such that $f(x)=f\left(x^{\prime}\right)$, the first phase transcripts for both $x$ and $x^{\prime}$ are identically distributed, that is, $\operatorname{transcript}\left(S^{1}(x), R^{*}\right) \equiv \operatorname{transcript}\left(S^{1}\left(x^{\prime}\right), R^{*}\right)$. Thus, if we fix a first-phase transcript $\tau \in \operatorname{transcript}\left(S^{1}(x), R^{*}\right)$ containing a value $y=f(x)$ in the reveal phase, any element in $\Gamma_{2, y}=f^{-1}(y) \subseteq \Gamma_{2}$ is equally likely to have generated $\tau$. Also observe that the $\Gamma_{2, y}$ 's form a partition of $\Gamma_{2}$.

Note that by definition, $\left|\Gamma_{2, y}\right| \geq 2^{n-t_{0}}$, and hence $\operatorname{CP}\left(\Gamma_{2, y}\right) \leq 2^{-\left(n-t_{0}\right)} \leq$ $2^{-\left(n-t_{0}-\Delta_{2}+3\right)}$. Therefore, we can apply Randomness Extraction Lemma, Lemma 6.6 to get $\mathrm{CP}^{1 / 2}\left(Z^{(2)}\right) \leq \sqrt{2^{-(q-1)}}$ for $Z^{(2)}=\left(H_{2}, H_{2}\left(\Gamma_{2, y}\right)\right)$.

Next, let $B_{\tau}=\operatorname{output}_{S}\left(S_{c}^{2}\left(\Gamma_{2, y}\right), R^{*}\right)(\tau)$ denote the private output of the sender $S$ in the second phase, which in turn is equal to the output of $S_{\mathrm{IH}}$ in the interactive hashing protocol, so equivalently $B_{\tau}=$ output $_{S_{\mathrm{IH}}}\left(S_{\mathrm{IH}}\left(Z^{(2)}\right), R^{*}\right)$. Similarly, let $W_{\tau}=$ view $_{R^{*}}\left(S_{c}^{2}\left(\Gamma_{2, y}\right), R^{*}\right)(\tau)$ denote the view of the adversarial receiver $R^{*}$ in the second phase, which in turn is equal to the view of $R^{*}$ in the interactive hashing protocol, so equivalently $W_{\tau}=\operatorname{view}_{R^{*}}\left(S_{\mathrm{IH}}\left(Z^{(2)}\right), R^{*}\right)$.

The final step is to use the hiding property of interactive hashing given by Lemma 6.9 to bound the collision probability of $B_{\tau}$ (the private output of the sender $S$ ) given $W_{\tau}$ (the view of the adversarial receiver $R^{*}$ ) as follows:

$$
\mathrm{CP}^{1 / 2}\left(B_{\tau} \mid W_{\tau}\right) \leq \sqrt{2^{q-k}} \cdot \sqrt{\mathrm{CP}\left(Z^{(2)}\right)} \leq \sqrt{2^{q-k}} \cdot \sqrt{2^{-(q-1)}}=\sqrt{2^{-(k-1)}} .
$$

6.2.4. Protocol 5.5 is 1 -out-of-2 binding in expected measure. The definition of 1-out-of-2 binding in Definition 5.4 considers the first phase (resp., second phase) to be broken if the sender $S^{*}$ produces valid decommitments to two different values after the first commit stage (resp., second commit stage). In this section and section 6.3 , we will work with a relaxed notion where we simply bound the expected number of values to which a cheating sender can open. To this end, we define openings $\left(S^{*}, R^{1}\right)$ (resp., openings $\left(S^{*}, R^{2}\right)$ ) to be a random variable denoting the set of values to which the sender successfully opens in phase 1 (resp., phase 2), where "successfully" opens is defined for each phase analogously to Definition 5.4. (Definition 5.4 refers to sender strategies that produce decommitments to one or two values in each reveal phase; here we consider a natural generalization where the sender tries to decommit to many values in each phase.) More formally, for a 2-phase commitment scheme $(S, R)$ and a "binding" set $\mathcal{B}$, we define openings $\left(S^{*}, R^{1}\right)(\mathcal{B})$ as follows:

- $S^{*}$ and $R_{c}^{1}$ interact to get first-phase commitment $c^{(1)}$.

- After the interaction, $S^{*}$ outputs a sequence of values $d_{1}^{(1)}, \ldots, d_{\ell}^{(1)}$ and corresponding full transcripts $\lambda_{1}, \ldots, \lambda_{\ell}$ of both phases. Recall that $\lambda_{i}=\left(\tau_{i}, \kappa_{i}\right)$, where $\tau_{i}$ and $\kappa_{i}$ are the first-phase and second-phase transcripts, respectively.

- We let openings $\left(S^{*}, R^{1}\right)(\mathcal{B})$ be the set of distinct values $d_{i}^{(1)}$ whose opening $\lambda_{i}$ is valid, where by valid we mean that $\lambda_{i}$ begins with prefix $c^{(1)}, \lambda_{i}$ contains a decommitment of $c^{(1)}$ to the value $d_{i}^{(1)}$ with first-phase transcript $\tau_{i} \notin \mathcal{B}$, and both $R_{r}^{1}$ and $R_{r}^{2}$ accept in $\lambda_{i}$.

Analogously, we define openings $\left(S^{*}, R^{2}\right)(\tau)$, where $\tau$ is a first-phase transcript, as follows: 
- $S^{*}$ and $R_{c}^{2}$ interact to get second-phase commitment $c^{(2)}$.

- After the interaction, $S^{*}$ outputs a sequence of values $d_{1}^{(2)}, \ldots, d_{\ell}^{(2)}$ and corresponding full transcripts $\lambda_{1}, \ldots, \lambda_{\ell}$.

- We let openings $\left(S^{*}, R^{2}\right)(\tau)$ be the set of distinct values $d_{i}^{(2)}$ whose opening $\lambda_{i}$ is valid, where by valid we mean that $\lambda_{i}$ starts with prefix $c^{(2)}$ (which in turn contains $\tau$ as a prefix), $\lambda_{i}$ contains a decommitment of $c^{(2)}$ to the value $d_{i}^{(2)}$, and $R_{r}^{2}$ accepts in $\lambda_{i}$.

Now, we can describe the binding property of Protocol 5.5 in this language (even when the underlying one-way function has unknown security).

Lemma 6.14 (Protocol 5.5 is 1-out-of-2 binding in expected measure). For every integer $t \in\{1,2, \ldots, n\}, k=O(\log n), \Delta_{1}=O(\log n)$, and $\Delta_{2}=O(\log n)$, the following holds for the 2-phase commitment scheme $(S, R)$ in Protocol 5.5 based on one-way function $f:\{0,1\}^{n} \rightarrow\{0,1\}^{n}$ :

There exists a binding set $\mathcal{B}$ for $(S, R)$ where the following hold.

(B.1) No PPT adversary $S^{*}$ can break the first-phase binding with nonnegligible probability in the sense of Definition 5.4. That is, for every PPT $S^{*}$, we have $\mid$ openings $\left(S^{*}, R^{1}\right)(\mathcal{B}) \mid \leq 1$ with probability $1-\operatorname{neg}(n)$ over the coins of $S^{*}$ and $R_{c}^{1}$.

(B.2) For all $\tau \in \mathcal{B}$ and every adversarial sender $S^{*}$,

$$
\mathrm{E}\left[\left|\operatorname{openings}\left(S^{*}, R^{2}\right)(\tau)\right|\right]<2,
$$

where the above expectation is taken over the coins of $S^{*}$ and $R^{2}$.

Proof. We follow the proof of the binding property in Lemma 5.9, using both Claims 5.11 and 5.10 from that proof. Let $\mathcal{B}=\left\{y \in\{0,1\}^{n}: \operatorname{Pr}\left[f\left(U_{n}\right)=y\right] \leq\right.$ $\left.2^{-t-\Delta_{3}}\right\}$ be the same binding set as defined in both claims. We set $\Delta_{3}=\Delta_{2}+$ $O(\log n)$ to be large enough so that the binding parameter poly $(n) \cdot 2^{-\Omega\left(\Delta_{3}-\Delta_{2}\right)} \cdot 2^{k / 2}$ in Claim 5.11 is at most $2^{-k}$. (This can be done since $k=O(\log n)$.) Now, Claim 5.11 states that if $\tau \in \mathcal{B}$, then the second commitment phase is binding. This implies that $\left|\operatorname{openings}\left(S^{*}, R^{2}\right)(\tau)\right| \geq 2$ with probability at most $2^{-k}$. Since $\mid$ openings $\left(S^{*}, R^{2}\right)(\tau) \mid \leq 2^{k}$ (the commitment is to a $k$-bit string), taking expectations we have

$$
\mathrm{E}\left[\mid \text { openings }\left(S^{*}, R^{2}\right)(\tau) \mid\right] \leq 2^{k} \cdot 2^{-k}+1 \cdot\left(1-2^{-k}\right)<2 .
$$

To see why property (B.1) holds, let $\varepsilon=\varepsilon(n)$ be the probability for which PPT $S^{*}$ breaks the first phase binding. Observe that the inversion success probability of $f$ from Claim 5.10 is

$$
\begin{aligned}
\varepsilon^{O(1)} \cdot 1 / \operatorname{poly}(n) \cdot 2^{-\left(k+\Delta_{1}+\Delta_{3}\right)} & =\varepsilon^{O(1)} \cdot 1 / \operatorname{poly}(n) \cdot 2^{-\left(k+\Delta_{1}+\Delta_{2}+O(\log n)\right)} \\
& =\frac{\varepsilon^{O(1)}}{\operatorname{poly}(n)},
\end{aligned}
$$

since all $k, \Delta_{1}, \Delta_{2}=O(\log n)$. This forces $\varepsilon(n)$ to be a negligible function.

6.3. Converting weakly hiding to strongly hiding commitments. In the previous section, we established that Protocol 5.5, with appropriate choice of parameters, is $1 / n$-hiding in $\mathrm{CP}^{1 / 2}$ measure (hence, only weakly hiding), and 1-out-of-2 binding in expected measure. Our goal in this section is to show how to boost the 
hiding probability to $\delta=1-\operatorname{neg}(n)$, therefore making the scheme strongly hiding, while maintaining the 1-out-of-2 binding property.

We first show how to double the hiding probability by combining a constant number of schemes to obtain a new scheme. We then repeat this doubling amplification process $O(\log n)$ times to boost the hiding probability from $1 / n$ to a constant $c>0$, hence obtaining an $\Omega(1)$-hiding scheme. Finally, we boost it all the way to $1-\operatorname{neg}(n)$ by combining polynomially many $\Omega(1)$-hiding schemes. This is all achieved via a hiding amplification procedure stated next. (See section 6.1 for an overview.)

Algorithm 6.15. Hiding amplification procedure, denoted as Amplify.

Input: 2-phase commitment $(S, R)$.

Additional Input Parameters: These are given in unary and are listed below:

1. Security parameter $n$.

2. Number $m$ of schemes $(S, R)$ to be combined.

3. Integer $r$ denoting $S$ 's private input length.

4. Integer $k$ denoting $S$ 's private output length.

5. Integer $k^{\prime}$ denoting $\mathbf{S}$ 's private output length.

6. Integer thresholds $\alpha_{1}$ and $\alpha_{2}$, for the first and second commit phases, respectively.

Output: 2-phase commitment $(\mathbf{S}, \mathbf{R})$, as described by Protocol 6.16 .

To reduce unnecessary clutter, we write $(\mathbf{S}, \mathbf{R})=\operatorname{Amplify}(S, R)$ when the rest of the parameters are clear from context.

Protocol 6.16. Amplified scheme $(\mathbf{S}, \mathbf{R})$ from hiding amplification of base scheme $(S, R)$.

Sender's private input: $x=\left(x_{1}, \ldots, x_{m}\right) \in\{0,1\}^{m r}$.

First-phase commit:

1. $\left(\mathbf{S}_{c}^{1}, \mathbf{R}_{c}^{1}\right)$ do $m$ sequential executions of $\left(S_{c}^{1}, R_{c}^{1}\right)$, using $x_{i}$ for $S_{c}^{1}$ 's private input in the ith execution. Let $\left(S_{c}^{1}[i]\left(x_{i}\right), R_{c}^{1}[i]\right)$ denote the ith execution of $\left(S_{c}^{1}, R_{c}^{1}\right)$. Define $a_{i}=$ output $_{S}\left(S_{c}^{1}[i]\left(x_{i}\right), R_{c}^{1}[i]\right) \in\{0,1\}^{k}$, and let $a=\left(a_{1}, \ldots, a_{m}\right)$.

2. Let $\mathcal{H}_{1}=\left\{h_{1}:\{0,1\}^{m k} \rightarrow\{0,1\}^{\alpha_{1}}\right\}$ be a family of pairwise-independent hash functions. $\mathbf{S}^{1}$ chooses a random hash $h_{1} \leftarrow \mathcal{H}_{1}$ and computes $y^{(1)}=\left(h_{1}, h_{1}(a)\right) \in\{0,1\}^{q}$.

3. $\left(\mathbf{S}_{c}^{1}, \mathbf{R}_{c}^{1}\right)$ run the interactive hashing protocol $\left(S_{\mathrm{IH}}^{1}\left(y^{(1)}\right), R_{\mathrm{IH}}^{1}\right)\left(1^{q}, 1^{k^{\prime}}\right)$, given by Protocol 3.3, with $\mathbf{S}^{1}$ and $\mathbf{R}^{1}$ acting as $S_{\mathrm{IH}}^{1}$ and $R_{\mathrm{IH}}^{1}$, respectively.

Let circuit $C:\{0,1\}^{k^{\prime}} \rightarrow\{0,1\}^{q}$ be the common output, and $d^{(1)} \in$ $\{0,1\}^{k^{\prime}}$ be $S_{\mathrm{IH}}^{1}$ 's private output in $\left(S_{\mathrm{IH}}^{1}\left(y^{(1)}\right), R_{\mathrm{IH}}^{1}\right)\left(1^{q}, 1^{k^{\prime}}\right)$.

First-phase sender's private output: String $d^{(1)} \in\{0,1\}^{k^{\prime}}$.

First-phase reveal:

$S_{r}^{1}$ sends tuple $\gamma^{(1)}=\left(d^{(1)}, a, h_{1}\right) \circ\left(\gamma_{1}^{(1)}, \ldots, \gamma_{m}^{(1)}\right)$, where $\gamma_{i}^{(1)}$ is the first phase revelation string of $S_{r}^{1}[i]$ in the above execution of $\left(S_{r}^{1}[i]\left(x_{i}\right), R_{r}^{1}[i]\right)$.

Receiver $\mathbf{R}_{r}^{1}$ accepts if and only if $C\left(d^{(1)}\right)=\left(h_{1}, h_{1}(a)\right)$ and $R_{r}^{1}[i]$ accepts $\left(\gamma_{i}^{(1)}, a_{i}\right)$ for all $i \in\{1,2, \ldots, m\}$.

Second-phase commit:

Second-phase common input: Transcript $\tau$ containing $\left(\tau_{1}, \ldots, \tau_{m}\right)$, where each $\tau_{i}=\operatorname{transcript}\left(S^{1}[i]\left(x_{i}\right), R^{1}[i]\right)$.

1. $\left(\mathbf{S}_{c}^{2}, \mathbf{R}_{c}^{2}\right)$ do $m$ sequential executions of $\left(S_{c}^{2}, R_{c}^{2}\right)$, using $x_{i}$ for $S^{2}$ 's secret and transcript $\tau_{i}$ in the ith execution. Let $\left(S_{c}^{2}[i]\left(x_{i}\right), R_{c}^{2}[i]\right)\left(\tau_{i}\right)$ denote the ith execution of $\left(S^{2}, R^{2}\right)$. Define $b_{i}=$ output $_{S}\left(S_{c}^{2}[i]\left(x_{i}\right), R_{c}^{2}[i]\right)\left(\tau_{i}\right) \in$ 
$\{0,1\}^{k}$, and let $b=\left(b_{1}, \ldots, b_{m}\right)$.

2. Let $\mathcal{H}_{2}=\left\{h_{2}:\{0,1\}^{m k} \rightarrow\{0,1\}^{\alpha_{2}}\right\}$ be a family of pairwise-independent hash functions. $\mathbf{S}^{2}$ chooses a random hash $h_{2} \leftarrow \mathcal{H}_{2}$ and computes $y^{(2)}=\left(h_{2}, h_{2}(b)\right) \in\{0,1\}^{q}$.

3. $\left(\mathbf{S}_{c}^{2}, \mathbf{R}_{c}^{2}\right)$ run the interactive hashing protocol $\left(S_{\mathrm{IH}}^{2}\left(y^{(2)}\right), R_{\mathrm{IH}}^{2}\right)\left(1^{q}, 1^{k^{\prime}}\right)$, given by Protocol 3.3, with $\mathbf{S}_{c}^{2}$ and $\mathbf{R}_{c}^{2}$ acting as $S_{\mathrm{IH}}^{2}$ and $R_{\mathrm{IH}}^{2}$, respectively.

Let circuit $C:\{0,1\}^{k^{\prime}} \rightarrow\{0,1\}^{q}$ be the common output and $d^{(2)} \in$ $\{0,1\}^{k^{\prime}}$ be $S_{\mathrm{IH}}^{2}$ 's private output in $\left(S_{\mathrm{IH}}^{2}\left(y^{(2)}\right), R_{\mathrm{IH}}^{2}\right)\left(1^{q}, 1^{k^{\prime}}\right)$.

Second-phase sender's private output: String $d^{(2)} \in\{0,1\}^{k^{\prime}}$.

Second-phase reveal:

$S_{r}^{2}$ sends tuple $\gamma^{(2)}=\left(d^{(2)}, b, h_{2}\right) \circ\left(\gamma_{1}^{(2)}, \ldots, \gamma_{m}^{(2)}\right)$, where $\gamma_{i}^{(2)}$ is the secondphase revelation string of $S_{r}^{2}[i]$ in the above execution of $\left(S_{r}^{2}[i]\left(x_{i}\right), R_{r}^{2}[i]\right)$.

Receiver $\mathbf{R}_{r}^{2}$ accepts if and only if $C^{(2)}\left(d^{(2)}\right)=\left(h_{2}, h_{2}(b)\right)$ and $R_{r}^{2}[i]$ accepts $\left(\gamma_{i}^{(2)}, b_{i}\right)$ for all $i \in\{1,2, \ldots, m\}$.

Starting from a weakly hiding scheme $\left(S_{0}, R_{0}\right)$ of Protocol 5.5 , we iteratively apply the amplification process Amplify, as described by Algorithm 6.17 below, to achieve a new scheme $(\mathrm{S}, \mathrm{R})$ that we will show to be statistically hiding. Let $D>1$ denote a large enough integer constant. We will set the number of schemes to be combined to be $m=D$ in all but the last iteration, in which we set $m=n$.

AlgORITHM 6.17. Iterative amplification procedure.

Input: Function $f:\{0,1\}^{n} \rightarrow\{0,1\}^{n}$, constant integer $D>1$, and thresholds $t \in$ $\{1,2, \ldots, n\}, \beta_{1}, \ldots, \beta_{\ell} \in\{0,1, \ldots, D-1\}, \beta_{\ell+1} \in\{0,1, \ldots, n\}$.

1. Let $\ell=\lceil\log n\rceil, k_{0}=(16 D) \cdot \ell$, and $\left(S_{0}, R_{0}\right)$ be the 2-phase commitment scheme based on function $f:\{0,1\}^{n} \rightarrow\{0,1\}^{n}$ from Protocol 5.5 using parameters $t, k=k_{0}$, and $\Delta_{1}=\Delta_{2}=\lceil 2 \log n\rceil$.

2. For $j=1,2, \ldots, \ell$, repeat the following:

(a) Set $k_{j}=k_{j-1}-8 D-8$.

(b) Set $\left(S_{j}, R_{j}\right)=\operatorname{Amplify}\left(S_{j-1}, R_{j-1}\right)$ for settings of parameters $m=$ $D, r=n \cdot D^{j-1}, k=k_{j-1}, k^{\prime}=k_{j}, \alpha_{1}=\left(\beta_{j}+1\right)\left(k_{j-1}-1\right)-3$, and $\alpha_{2}=\left(D-\beta_{j}\right)\left(k_{j-1}-1\right)-3$.

3. Set $(\mathrm{S}, \mathrm{R})=\operatorname{Amplify}\left(S_{\ell}, R_{\ell}\right)$ for settings of parameters $m=n, r=n \cdot D^{\ell}$, $k=k_{\ell}, k^{\prime}=n, \alpha_{1}=\left\lfloor\left(\beta_{\ell+1}+\frac{1}{3} \delta n\right) k\right\rfloor$, and $\alpha_{2}=\left\lfloor\left(n-\beta_{\ell+1}+\frac{1}{3} \delta n\right) k\right\rfloor$, where $\delta=1 / D$.

Output: 2-phase commitment scheme $(\mathrm{S}, \mathrm{R})$.

Lemma 6.18. If scheme $\left(S_{0}, R_{0}\right)$ used by Algorithm 6.17 runs in polynomial time, then scheme $(\mathrm{S}, \mathrm{R})$, the output of Algorithm 6.17, also runs in polynomial time.

Proof. Scheme $(\mathrm{S}, \mathrm{R})$ consists of $n \cdot D^{\ell}=n \cdot D^{O(\log n)}=\operatorname{poly}(n)$ executions of $\left(S_{0}, R_{0}\right)$. In addition, each amplification procedure Amplify adds an overhead time of poly $(n)$ since both the sender and receiver are doing interactive hashing. Since there are only $1+n+n D+n D^{2}+\cdots+D^{\ell-1}=\operatorname{poly}(n)$ amplification steps, the overhead time is polynomial. Hence, scheme $(\mathrm{S}, \mathrm{R})$ runs in polynomial time if $\left(S_{0}, R_{0}\right)$ does, too.

The rest of this section, which is technically involved, is devoted to proving the hiding and binding properties of the final scheme $(S, R)$. (In process, we also analyze the hiding and binding properties of intermediate schemes $\left(S_{j}, R_{j}\right)$.)

6.3.1. Hiding amplification. The following two lemmas, Lemmas 6.19 and 6.20 , provide us with a way to understand the hiding property (in the $\mathrm{CP}^{1 / 2}$ measure) of amplified scheme $(\mathbf{S}, \mathbf{R})$ in terms of its base scheme $(S, R)$. Lemma 6.19 basically 
says that the hiding probability doubles when we go from $\left(S_{j-1}, R_{j-1}\right)$ to $\left(S_{j}, R_{j}\right)=$ Amplify $\left(S_{j-1}, R_{j-1}\right)$ (refer to step 2(b) in Algorithm 6.17). So if we start up with a $1 / n$-hiding scheme $\left(S_{0}, R_{0}\right)$, in $\ell=\log n$ iterations, we will get a scheme $\left(S_{\ell}, R_{\ell}\right)$ with $\Omega(1)$-hiding. Lemma 6.20 essentially argues that the final amplification step boosts the hiding probability all the way to $1-\operatorname{neg}(n)$ (in both phases) when starting from a scheme that is $\Omega(1)$-hiding. With these two lemmas, we can establish that the final scheme $(\mathrm{S}, \mathrm{R})=\operatorname{Amplify}\left(S_{\ell}, R_{\ell}\right)$ is statistically hiding in both phases.

LEMma 6.19 (intermediate step hiding amplification). For every sufficiently large constant $D \in \mathbb{Z}^{+}$, the following holds: If scheme $(S, R)$ is $\delta$-hiding in $\mathrm{CP}^{1 / 2}$ measure, then there exists an integer $\beta \in\{0,1, \ldots, D-1\}$ such that scheme $(\mathbf{S}, \mathbf{R})=$ $\operatorname{Amplify}(S, R)$, with parameters $m=D, k^{\prime}=k-8 D-8, \alpha_{1}=(\beta+1)(k-1)-3$, and $\alpha_{2}=(D-\beta)(k-1)-3$, is $\delta^{\prime}$-hiding in $\mathrm{CP}^{1 / 2}$ measure for $\delta^{\prime}=\min \{2 \delta, 1 / D\}$.

Proof. Without loss of generality, we may assume that $R^{*}$ is deterministic since we can fix the coins of $R^{*}$ that maximize the collision probability. Throughout this proof, the value of $m$ will be fixed to $D$, although we will keep writing $m$. Let the $\delta$-hiding properties, as stated in Definition 6.11, of $(S, R)$ be (H.1), (H.2), and (H.3), respectively. We will prove that $(\mathbf{S}, \mathbf{R})$ satisfies Definition 6.11 with Properties $\left(\mathrm{H}^{\prime} .1\right)$, $\left(\mathrm{H}^{\prime} .2\right)$, and $\left(\mathrm{H}^{\prime} .3\right)$ by showing that Property $(\mathrm{H} .1)$ implies $\left(\mathrm{H}^{\prime} .1\right)$, and so forth.

Property (H.1) implies $\left(\mathrm{H}^{\prime} .1\right)$. Let $\Gamma_{1}$ and $\Gamma_{2}$ be the corresponding sets for $(S, R)$. Define the sets $\Gamma_{1}^{\prime}$ and $\Gamma_{2}^{\prime}$ in terms as follows (the value of $\beta$ will be determined later):

$$
\begin{aligned}
& \Gamma_{1}^{\prime}=\left\{\left(x_{1}, \ldots, x_{m}\right): \exists i_{1}, \ldots, i_{\beta+1} \text { such that } x_{i_{1}}, \ldots, x_{i_{\beta+1}} \in \Gamma_{1}\right\}, \\
& \Gamma_{2}^{\prime}=\left\{\left(x_{1}, \ldots, x_{m}\right): \exists i_{1}, \ldots, i_{m-\beta} \text { such that } x_{i_{1}}, \ldots, x_{i_{m-\beta}} \in \Gamma_{2}\right\} .
\end{aligned}
$$

By the way we defined $\Gamma_{1}^{\prime}$ and $\Gamma_{2}^{\prime}$ together with the fact that $\Gamma_{1} \cup \Gamma_{2}=\{0,1\}^{r}$, it is the case that $\Gamma_{1}^{\prime} \cup \Gamma_{2}^{\prime}=\{0,1\}^{m r}$. This is because either at least $\beta+1$ of the $x_{i}$ are in $\Gamma_{1}$ (in which case, $\left.\left(x_{1}, \ldots, x_{m}\right) \in \Gamma_{1}^{\prime}\right)$ or else at most $\beta$ of the $x_{i}$ are in $\Gamma_{1}$, which implies that at least $m-\beta$ of the $x_{i}$ are in $\Gamma_{2}$ (in which case $\left(x_{1}, \ldots, x_{m}\right) \in \Gamma_{2}^{\prime}$ ).

We are given that $\mu\left(\Gamma_{1} \cap \Gamma_{2}\right) \geq \delta$. Define $\delta^{\prime}=\min \{\delta, 1 /(2 m)\}$. What we need to show is that $\mu\left(\Gamma_{1}^{\prime} \cap \Gamma_{2}^{\prime}\right) \geq \delta^{\prime}$. Choose any subset $S \subseteq \Gamma_{1} \cap \Gamma_{2}$ such that $\mu(S)=\delta^{\prime}$. Hence, we have

$$
\begin{aligned}
\operatorname{xin}_{1, \ldots, x_{m} \leftarrow\{0,1\}^{r}}\left[\text { exactly one } x_{i} \in S\right] & =m \delta^{\prime}\left(1-\delta^{\prime}\right)^{m-1} \geq m \delta^{\prime}(1-1 /(m-1))^{m-1} \\
& =\Omega\left(m \delta^{\prime}\right) .
\end{aligned}
$$

Given that exactly one $x_{i} \in S$, assume without loss of generality that $x_{m} \in S$. Let $p_{t}$ denote the conditional probability that exactly $t$ of the rest of the $m-1 x_{i}$ 's are in $\Gamma_{1} \backslash \Gamma_{2}$. Choose $\beta \in[0, m-1]$ to maximize $p_{t}$, i.e., $\beta=\operatorname{argmax}_{t} p_{t}$. Let $I_{i}$ for $i=1,2, \ldots, m-1$ be a binary random variable indicating whether $x_{i} \in \Gamma_{1}$ or not; note that these are independent random variables conditioned on the fact that $x_{m} \in S$. Let $\mu$ be the mean of the $I_{i}$ 's. By a Chernoff bound,

$$
\operatorname{Pr}\left[\left|\sum_{i} I_{i}-\mu \cdot(m-1)\right|>3 \sqrt{m-1}\right] \leq 2 e^{((m-1) / 3) \cdot(3 / \sqrt{m-1})^{2}}<1 / 2 .
$$

This means that greater than half of the probability mass is in the interval $\mu \cdot(m-$ $1) \pm 3 \sqrt{m-1}$. Since we chose $\beta=\operatorname{argmax}_{t} p_{t}$ in a maximal way, we have

$$
\underset{x_{1}, \ldots, x_{m} \leftarrow\{0,1\}^{r}}{\operatorname{Pr}}\left[\text { exactly } \beta \text { of } x_{i} \text { 's are in } \Gamma_{1} \backslash S \mid \text { exactly one } x_{i} \in S\right]=\Omega\left(\frac{1}{\sqrt{m}}\right) .
$$


Knowing that $\Gamma_{1} \cup \Gamma_{2}=\{0,1\}^{r}$, if exactly $\beta$ of $x_{i}$ 's in $\Gamma_{1} \backslash S$ and exactly one $x_{i} \in S$, then there must be at least $m-1-\beta$ of $x_{i}$ 's in $\Gamma_{2} \backslash S$. Consequently,

$$
\begin{aligned}
\operatorname{Pr}_{x_{1}, \ldots, x_{m} \leftarrow\{0,1\}^{r}}\left[\left(x_{1}, \ldots, x_{m}\right) \in \Gamma_{1}^{\prime} \cap \Gamma_{2}^{\prime}\right] & =\Omega\left(m \delta^{\prime}\right) \cdot \Omega\left(\frac{1}{\sqrt{m}}\right) \\
& =\Omega\left(\sqrt{m} \delta^{\prime}\right) \\
& >2 \delta^{\prime}=\min \{2 \delta, 1 / m\},
\end{aligned}
$$

where the last inequality holds when $m=D$ is a large enough constant.

Property (H.2) implies $\left(\mathrm{H}^{\prime} .2\right)$. In the first commitment phase $\left(\mathbf{S}_{c}^{1}, R^{*}\right)$, the cheating receiver $R^{*}$ interacts with $m$ sequential executions of $S_{c}^{1}$. Here we must analyze the case when $S_{c}^{1}$ 's private input in these $m$ executions, given by $x=\left(x_{1}, \ldots, x_{m}\right)$, is distributed uniformly in $\Gamma_{1}^{\prime}$. We let $A_{i}(x)$ denote the private output of the sender and $V_{i}(x)$ the view of the receiver in the $i$ th execution, for $x$ being the private input for $\mathbf{S}_{c}^{1}$. That is, for $i=1, \ldots, m$,

$$
\begin{gathered}
A_{i}(x)=\operatorname{output}_{S}\left(S_{c}^{1}\left(x_{i}\right), R^{*}\left(V_{1}, \ldots, V_{i-1}\right)\right) ; \\
V_{i}(x)=\operatorname{view}_{R^{*}}\left(S_{c}^{1}\left(x_{i}\right), R^{*}\left(V_{1}, \ldots, V_{i-1}\right)\right) .
\end{gathered}
$$

Note that while the sender's behavior in the $i$ th execution is independent of the previous executions, the cheating receiver may base its strategy on its previous views. We want to bound $\mathrm{CP}^{1 / 2}\left(A^{\prime \prime}\left(\Gamma_{1}^{\prime}\right) \mid V^{\prime \prime}\left(\Gamma_{1}^{\prime}\right)\right)$, where $A^{\prime \prime}\left(\Gamma_{1}^{\prime}\right)=\left(A_{1}\left(\Gamma_{1}^{\prime}\right), \ldots, A_{m}\left(\Gamma_{1}^{\prime}\right)\right)$ represents the combined first-phase private outputs of the $m$ senders, and $V^{\prime \prime}\left(\Gamma_{1}^{\prime}\right)=$ $\left(V_{1}\left(\Gamma_{1}^{\prime}\right), \ldots, V_{m}\left(\Gamma_{1}^{\prime}\right)\right)$ represents the view of $R^{*}$ when interacting with these $m$ senders. Note that random variable $\Gamma_{1}^{\prime}$ represents an independent random element from the set $\Gamma_{1}^{\prime}$. To do this, we consider, for each $I \subseteq[m]$ of size at least $\beta+1$, the random variable $\left.\Gamma_{1}^{\prime}\right|_{I}$ for private input of the sender $\mathbf{S}$, where $\left.\Gamma_{1}^{\prime}\right|_{I}$ represents choosing $x_{i}$ uniformly in $\Gamma_{1}$ for $i \in I$, and uniformly in $\overline{\Gamma_{1}}$ for $i \notin I$. To get a bound on $\mathrm{CP}^{1 / 2}\left(A^{\prime \prime}\left(\left.\Gamma_{1}^{\prime}\right|_{I}\right) \mid V^{\prime \prime}\left(\left.\Gamma_{1}^{\prime}\right|_{I}\right)\right)$, we will have to refer to Lemma 6.5 and see why the $\left(A_{i}, V_{i}\right)$ 's satisfy the two conditions of the lemma.

Conditioned on the any previous view-namely, $V_{1}\left(\left.\Gamma_{1}^{\prime}\right|_{I}\right)=v_{1}, \ldots, V_{i-1}\left(\left.\Gamma_{1}^{\prime}\right|_{I}\right)=$ $v_{i-1}$ for any $v_{1}, \ldots, v_{i-1}$ it is the case that $\mathrm{CP}^{1 / 2}\left(A_{i}\left(\left.\Gamma_{1}^{\prime}\right|_{I}\right) \mid V_{i}\left(\left.\Gamma_{1}^{\prime}\right|_{I}\right)\right) \leq \sqrt{2^{-(k-1)}}$ if $i \in I$. This follows from Property (H.2) because the receiver $R^{*}$ can incorporate the previous view $v_{1}, \ldots, v_{i-1}$ as advice (since $R^{*}$ is unbounded), and then the only randomness in the definition of $A_{i}$ and $V_{i}$ is the sender's coins $x_{i} \leftarrow\left(\left.\Gamma_{1}^{\prime}\right|_{I}\right)_{i}$, which are uniform in $\Gamma_{1}$ (even conditioned on $v_{1}, \ldots, v_{i-1}$ ). This shows that the first condition of Lemma 6.5 is satisfied.

For the second condition, what we need to show is that conditioned on $V_{1}\left(\left.\Gamma_{1}^{\prime}\right|_{I}\right)=$ $v_{1}, \ldots, V_{i}\left(\left.\Gamma_{1}^{\prime}\right|_{I}\right)=v_{i}$, the random variables $A_{1}\left(\left.\Gamma_{1}^{\prime}\right|_{I}\right), \ldots, A_{i}\left(\left.\Gamma_{1}^{\prime}\right|_{I}\right), V_{i+1}\left(\left.\Gamma_{1}^{\prime}\right|_{I}\right)$ are independent. This can be seen by induction on $i$ as follows. It is vacuously true for $i=0$. Assuming it is true for $i=j-1$, we prove it for $i=j$ as follows. First condition on $v_{1}, \ldots, v_{j-1}$. By inductive hypothesis, $A_{1}, \ldots, A_{j-1}, V_{j}$ are independent (omitting $\left.\Gamma_{1}^{\prime}\right|_{I}$ from the notation for readability). Moreover, since we have conditioned on $v_{1}, \ldots, v_{j-1}$, we have that $A_{j}$ and $V_{j}$ are functions of only $\left(\left.\Gamma_{1}^{\prime}\right|_{I}\right)_{j}$, the sender's coins in the $j$ th execution, which is independent of $A_{1}, \ldots, A_{j-1}$ (because we have used only $\left(\left.\Gamma_{1}^{\prime}\right|_{I}\right)_{1}, \ldots,\left(\left.\Gamma_{1}^{\prime}\right|_{I}\right)_{j-1}$ so far). Thus, if we condition on $V_{j}=v_{j}, A_{j}$ remains independent of $A_{1}, \ldots, A_{j-1} . V_{j+1}$ is independent of $A_{1}, \ldots, A_{j}$ because now it is only a function of $\left(\left.\Gamma_{1}^{\prime}\right|_{I}\right)_{j+1}$, which has not been used yet.

Applying Lemma 6.5, we have

$$
\mathrm{CP}^{1 / 2}\left(A^{\prime \prime}\left(\left.\Gamma_{1}^{\prime}\right|_{I}\right) \mid V^{\prime \prime}\left(\left.\Gamma_{1}^{\prime}\right|_{I}\right)\right) \leq \sqrt{2^{-(\beta+1)(k-1)}},
$$


since from property (H.2) it is the case that for all $i \in I, \mathrm{CP}^{1 / 2}\left(A_{i} \mid V_{i}\right) \leq \sqrt{2^{-(k-1)}}$ (even conditioned on the previous views), and $|I| \geq \beta+1$.

Now, to bound $\mathrm{CP}^{1 / 2}\left(A^{\prime \prime}\left(\Gamma_{1}^{\prime}\right) \mid V^{\prime \prime}\left(\Gamma_{1}^{\prime}\right)\right)$ where $X$ is uniform in $\Gamma_{1}^{\prime}$, we observe that $\Gamma_{1}^{\prime}=\left.\Gamma_{1}^{\prime}\right|_{\mathcal{I}}$, where $\mathcal{I}$ is the random variable on subsets $I$ of size at least $\beta+1$ given by

$$
\operatorname{Pr}[\mathcal{I}=I]=\underset{\left(x_{1}, \ldots, x_{m}\right) \leftarrow \Gamma_{1}^{\prime}}{\operatorname{Pr}}\left[\left\{i: x_{i} \in \Gamma_{1}\right\}=I\right] .
$$

In other words, sampling from $\Gamma_{1}^{\prime}$ can be broken into two steps; first sampling an $I \leftarrow \mathcal{I}$, and then sampling $x_{i} \leftarrow \Gamma_{1}$ for $i \in I$, and $x_{i} \leftarrow \overline{\Gamma_{1}}$ for $i \notin I$. Therefore, we have

$$
\begin{aligned}
\mathrm{CP}^{1 / 2}\left(A^{\prime \prime}\left(\left.\Gamma_{1}^{\prime}\right|_{\mathcal{I}}\right) \mid V^{\prime \prime}\left(\left.\Gamma_{1}^{\prime}\right|_{\mathcal{I}}\right)\right) & \leq \mathrm{CP}^{1 / 2}\left(A^{\prime \prime}\left(\left.\Gamma_{1}^{\prime}\right|_{\mathcal{I}}\right) \mid\left(V^{\prime \prime}\left(\left.\Gamma_{1}^{\prime}\right|_{\mathcal{I}}\right), \mathcal{I}\right)\right) \quad \text { (by Lemma 6.7) } \\
& =\underset{I \leftarrow \mathcal{I}}{\mathrm{E}}\left[\mathrm{CP}^{1 / 2}\left(A^{\prime \prime}\left(\left.\Gamma_{1}^{\prime}\right|_{I}\right) \mid V^{\prime \prime}\left(\left.\Gamma_{1}^{\prime}\right|_{I}\right)\right)\right] \\
& \leq \sqrt{2^{-(\beta+1)(k-1)}} \\
& =\sqrt{2^{-\left(\alpha_{1}+3\right)}}
\end{aligned}
$$

with the last inequality following from (3). Therefore we can apply the Randomness Extraction Lemma, Lemma 6.6, to get $\mathrm{CP}^{1 / 2}\left(H_{1}, H_{1}\left(A^{\prime \prime}\left(\Gamma_{1}^{\prime}\right)\right) \mid V^{\prime \prime}\left(\Gamma_{1}^{\prime}\right)\right) \leq \sqrt{2^{-(q-1)}}$, where $H_{1}$ is an independent random hash from $\mathcal{H}_{1}$.

Next, let $A^{\prime}=$ output $_{\mathbf{S}}\left(\mathbf{S}^{1}\left(\Gamma_{1}^{\prime}\right), R^{*}\right)$ denote the private output of the sender $\mathbf{S}$ in the first phase, which in turn is equal to the output of $S_{\mathrm{IH}}$ in the interactive hashing protocol, so equivalently $A^{\prime}=$ output $_{S_{\mathrm{IH}}}\left(S_{\mathrm{IH}}(Q), R_{\mathrm{IH}}^{*}\right)$, where $Q=\left(H_{1}, H_{1}\left(A^{\prime \prime}\left(\Gamma_{1}^{\prime}\right)\right)\right)$. Similarly, let $V^{\prime}=\operatorname{view}_{R^{*}}\left(\mathbf{S}^{1}\left(\Gamma_{1}^{\prime}\right), R^{*}\right)$ denote the view of the adversarial receiver $R^{*}$ in the first phase, which in turn is equal to the view of $R^{*}$ in the interactive hashing protocol together with $V^{\prime \prime}$, so equivalently $V^{\prime}=\left(\operatorname{view}_{R_{\mathrm{IH}}^{*}}\left(S_{\mathrm{IH}}(Q), R_{\mathrm{IH}}^{*}\right), V^{\prime \prime}\right)$ for the same $Q=\left(H_{1}, H_{1}\left(A^{\prime \prime}\left(\Gamma_{1}^{\prime}\right)\right)\right)$.

The final step is to use the hiding property of interactive hashing given by Lemma 6.9 to bound the collision probability of $A^{\prime}$ (the private output of the sender S) given $V^{\prime}$ (the view of the adversarial receiver $R^{*}$ ) as follows:

$$
\mathrm{CP}^{1 / 2}\left(A^{\prime} \mid V^{\prime}\right) \leq \sqrt{2^{q-k^{\prime}}} \cdot \mathrm{CP}^{1 / 2}\left(Q \mid V^{\prime \prime}\right) \leq \sqrt{2^{q-k^{\prime}}} \cdot \sqrt{2^{-(q-1)}}=\sqrt{2^{-\left(k^{\prime}-1\right)}} .
$$

Property (H.3) implies $\left(\mathrm{H}^{\prime} .3\right)$. Fix a transcript $\tau^{\prime} \in \operatorname{Supp}\left(\mathrm{T}^{\prime}\right)$, where random variable $\mathrm{T}^{\prime}=\operatorname{transcript}\left(\mathbf{S}^{1}\left(\Gamma_{2}^{\prime}\right), R^{*}\right)$. Transcript $\tau^{\prime}$ contains first-phase transcripts $\left(\tau_{1}, \ldots, \tau_{m}\right)$ for the $m$ executions of $(S, R)$. Similarly to the above proof of Property $\left(\mathrm{H}^{\prime} .2\right)$, we define the following random variables:

$$
\begin{aligned}
B_{i}(x) & =\operatorname{output}_{S}\left(S_{c}^{2}\left(x_{i}\right), R^{*}\left(W_{1}, \ldots, W_{i-1}\right)\left(\tau_{i}\right)\right), \\
W_{i}(x) & =\operatorname{view}_{R^{*}}\left(S_{c}^{2}\left(x_{i}\right), R^{*}\left(W_{1}, \ldots, W_{i-1}\right)\left(\tau_{i}\right)\right),
\end{aligned}
$$

where $x_{i}$ is the private input of the sender in the $i$ th execution of the $(S, R)$. As above, we want to bound $\mathrm{CP}^{1 / 2}\left(B^{\prime \prime}\left(X_{\tau^{\prime}}\right) \mid W^{\prime \prime}\left(X_{\tau^{\prime}}\right)\right)$, where random variable $B^{\prime \prime}\left(X_{\tau^{\prime}}\right)=$ $\left(B_{1}\left(X_{\tau^{\prime}}\right), \ldots, B_{m}\left(X_{\tau^{\prime}}\right)\right)$ represents the combined second-phase private outputs of the $m$ senders, and random variable $W^{\prime \prime}\left(X_{\tau^{\prime}}\right)=\left(W_{1}\left(X_{\tau^{\prime}}\right), \ldots, W_{m}\left(X_{\tau^{\prime}}\right)\right)$ represents the view of $R^{*}$ when interacting with these $m$ senders, with $X_{\tau^{\prime}}$ being a shorthand for $\left.\Gamma_{2}^{\prime}\right|_{\mathrm{T}\left(\Gamma_{2}^{\prime}\right)=\tau^{\prime}}$. To do this, we consider, for each subset $J \subseteq[m]$ of size at least $m-\beta$, the random variable $X_{J}$ for private input of the sender $\mathbf{S}$, where $X_{J}$ represents choosing $x_{i}$ uniformly in $\Gamma_{2}$ for $i \in J$ and uniformly in $\overline{\Gamma_{2}}$ for $i \notin J$. 
It is important to note that even when we condition on $\mathrm{T}^{\prime}\left(X_{J}\right)=\tau^{\prime}$, the components $\left(X_{1}, \ldots, X_{m}\right)$ of $X_{J}$ remain independent, and the distribution of $\left.X_{i}\right|_{\mathrm{T}^{\prime}\left(X_{J}\right)=\tau^{\prime}}$ is equivalent to $\left.X_{i}\right|_{\mathrm{T}\left(X_{i}\right)=\tau_{i}}$, where we condition only on the transcript of the $i$ th execution. (Similarly to the inductive proof above, it can be shown that $\left(X_{1}, \ldots, X_{m}\right)$ are independent given the receiver's view $V_{m}$ of the $m$ executions of $S_{c}^{1}$. The only additional information revealed about the $X_{i}$ 's in the first phase is $\left(A_{1}, \ldots, A_{m}\right)$, where $A_{i}$ is a function only of $X_{i}$ once we condition on $V_{m}$.)

Thus from property (H.3), we have for all $i \in J, \mathrm{CP}^{1 / 2}\left(B_{i}\left(X_{J, \tau^{\prime}}\right) \mid W_{i}\left(X_{J, \tau^{\prime}}\right)\right) \leq$ $\sqrt{2^{-(k-1)}}$, where $X_{J, \tau^{\prime}}=\left.\left.\Gamma_{2}^{\prime}\right|_{J}\right|_{\mathrm{T}^{\prime}\left(\left.\Gamma_{2}^{\prime}\right|_{J}\right)=\tau^{\prime}}$, and this holds even conditioned on the previous views. Similarly to the first phase, we apply Lemma 6.5 to show that

$$
\mathrm{CP}^{1 / 2}\left(B^{\prime \prime}\left(X_{J, \tau^{\prime}}\right) \mid W^{\prime \prime}\left(X_{J, \tau^{\prime}}\right)\right) \leq \sqrt{2^{-(m-\beta)(k-1)}} .
$$

Again, analogously to the first phase, we observe that $X_{\tau^{\prime}}=X_{\mathcal{J}, \tau^{\prime}}$ for an appropriate random variable $\mathcal{J}$ on sets of size at least $m-\beta$, and thus

$$
\begin{aligned}
\mathrm{CP}^{1 / 2}\left(B^{\prime \prime}\left(X_{\tau^{\prime}}\right) \mid W^{\prime \prime}\left(X_{\tau^{\prime}}\right)\right) & \leq \sqrt{2^{-(m-\beta)(k-1)}} \\
& =\sqrt{2^{-\left(\alpha_{2}+3\right)}} .
\end{aligned}
$$

By the Randomness Extraction Lemma, Lemma 6.6, we get

$$
\mathrm{CP}^{1 / 2}\left(H_{2}, H_{2}\left(B^{\prime \prime}\left(X_{\tau^{\prime}}\right)\right) \mid W^{\prime \prime}\left(X_{\tau^{\prime}}\right)\right) \leq \sqrt{2^{-(q-1)}} .
$$

The final step is to use the hiding property of interactive hashing given by Lemma 6.9 to bound the collision probability of $B_{\tau}$ (the private output of the sender $S$ ) given $W_{\tau}$ (the view of the adversarial receiver $R^{*}$ ) as follows:

$$
\mathrm{CP}^{1 / 2}\left(B_{\tau^{\prime}}^{\prime} \mid W_{\tau^{\prime}}^{\prime}\right) \leq \sqrt{2^{q-k^{\prime}}} \cdot \sqrt{2^{-(q-1)}}=\sqrt{2^{-\left(k^{\prime}-1\right)}} .
$$

Lemma 6.20 (final step hiding amplification). The following statement holds for every constant $\delta>0$ and every integer $k \geq 100 / \delta$ : If scheme $(S, R)$ is $\delta$ hiding in $\mathrm{CP}^{1 / 2}$ measure, then there exists an integer $\beta \in[0, n]$ such that scheme $(\mathbf{S}, \mathbf{R})=\operatorname{Amplify}(S, R)$, with parameters $m=n, k^{\prime}=n, \alpha_{1}=\left\lfloor\left(\beta+\frac{1}{3} \delta n\right) k\right\rfloor$, and $\alpha_{2}=\left\lfloor\left(n-\beta+\frac{1}{3} \delta n\right) k\right\rfloor$, is statistically hiding in the sense of Definition 5.3.

Proof. Let the $\delta$-hiding properties, as stated in Definition 6.11, of $(S, R)$ be (H.1), (H.2), and (H.3), respectively. To prove that scheme (S, R) is statistically hiding, it suffices to show that there exist sets $\Gamma_{1}^{\prime}, \Gamma_{2}^{\prime} \subseteq\{0,1\}^{n r}$ such that the following hold for every adversarial receiver $R^{*}$ :

$\left(\mathrm{H}^{\prime} .1\right)$ Both $\mu\left(\Gamma_{1}^{\prime}\right), \mu\left(\Gamma_{2}^{\prime}\right) \geq 1-2^{-\Omega(n)}$.

$\left(\mathrm{H}^{\prime} .2\right)\left(A^{\prime}, V^{\prime}\right)$ is $2^{-\Omega(n)}$-close to $\left(U_{n}, V^{\prime}\right)$, where $A^{\prime}=\operatorname{output}_{\mathbf{S}}\left(\mathbf{S}_{c}^{1}\left(\Gamma_{1}^{\prime}\right), R^{*}\right)$ denotes the private output of the sender $\mathbf{S}$ in the first phase, and $V^{\prime}=\operatorname{view}_{R^{*}}\left(\mathbf{S}_{c}^{1}\left(\Gamma_{1}^{\prime}\right), R^{*}\right)$ denotes the view of the adversarial receiver $R^{*}$ in the first phase.

$\left(\mathrm{H}^{\prime} .3\right)$ For all $\tau^{\prime} \in \operatorname{Supp}\left(\mathrm{T}^{\prime}\right),\left(B_{\tau^{\prime}}^{\prime}, W_{\tau^{\prime}}^{\prime}\right)$ is $2^{-\Omega(n)}$-close to $\left(U_{n}, W_{\tau^{\prime}}^{\prime}\right)$, where random variable $\left(B_{\tau^{\prime}}^{\prime}, W_{\tau^{\prime}}^{\prime}\right)=\operatorname{output}_{\mathbf{S}}\left(\mathbf{S}_{c}^{2}\left(\Gamma_{2}^{\prime}\right), R^{*}\right)$, view $\left._{R^{*}}\left(\mathbf{S}_{c}^{2}\left(\Gamma_{2}^{\prime}\right), \quad R^{*}\right)\right)\left.\right|_{\mathrm{T}^{\prime}=\tau^{\prime}}$, and random variable $\mathrm{T}^{\prime}=$ transcript $\left(\mathbf{S}^{1}\left(\Gamma_{2}^{\prime}\right), R^{*}\right)$. In other words, $B_{\tau^{\prime}}^{\prime}$ is the private output of the sender $\mathbf{S}$ in the second phase given that the first-phase transcript is $\tau^{\prime}$ and $W_{\tau^{\prime}}^{\prime}$ is the view of the adversarial receiver $R^{*}$ in the second phase given that the first-phase transcript is $\tau^{\prime}$. 
Property (H.1) implies $\left(\mathrm{H}^{\prime} .1\right)$. Let $\Gamma_{1}$ and $\Gamma_{2}$ be the corresponding sets for $(S, R)$, and let $p=\mu\left(\Gamma_{1}\right)$. Set $\beta=\left\lfloor p n-\frac{1}{2} \delta n\right\rfloor, \gamma_{1}=\left\lfloor p n-\frac{1}{12} \delta n\right\rfloor$, and $\gamma_{2}=\lfloor(1-p+\delta) n-$ $\left.\frac{1}{12} \delta n\right\rfloor$. Note that $\beta \in[0, n]$ since $p \in[\delta, 1]$.

Define the sets $\Gamma_{1}^{\prime}$ and $\Gamma_{2}^{\prime}$ as follows:

$$
\begin{aligned}
& \Gamma_{1}^{\prime}=\left\{\left(x_{1}, \ldots, x_{n}\right): \exists i_{1}, \ldots, i_{\gamma_{1}} \text { such that } x_{i_{1}}, \ldots, x_{i_{\gamma_{1}}} \in \Gamma_{1}\right\}, \\
& \Gamma_{2}^{\prime}=\left\{\left(x_{1}, \ldots, x_{n}\right): \exists i_{1}, \ldots, i_{\gamma_{2}} \text { such that } x_{i_{1}}, \ldots, x_{i_{\gamma_{2}}} \in \Gamma_{2}\right\} .
\end{aligned}
$$

To lower bound $\mu\left(\Gamma_{1}^{\prime}\right)$, note that $\mu\left(\Gamma_{1}\right)-\gamma_{1} / n=p-\left\lfloor p n-\frac{1}{12} \delta n\right\rfloor / n \geq \frac{1}{12} \delta=\Omega(1)$ since $\delta=\Omega(1)$. Using a Chernoff bound, we get

$$
\begin{aligned}
\mu\left(\Gamma_{1}^{\prime}\right) & =1-\underset{\left(x_{1}, \ldots, x_{n}\right)}{\operatorname{Pr}}\left[\text { fewer than } \gamma_{1} \text { of the } x_{i} \text { 's are in } \Gamma_{1}\right] \\
& =1-2^{-\Omega(n)} .
\end{aligned}
$$

To analyze $\mu\left(\Gamma_{2}^{\prime}\right)$, we note that $\mu\left(\Gamma_{2}\right)-\gamma_{2} / n \geq(1-p+\delta)-\left\lfloor(1-p+\delta) n-\frac{1}{12} \delta n\right\rfloor / n$ $\geq \frac{1}{12} \delta=\Omega(1)$. Using ananalysis similar to that above, we get $\mu\left(\Gamma_{2}^{\prime}\right)=1-2^{-\Omega(n)}$.

Property (H.2) implies $\left(\mathrm{H}^{\prime} .2\right)$. Using the same notation and analysis as in the proof of Lemma 6.19 , we let $A_{i}(x)$ denote the private output of the sender and $V_{i}(x)$ the view of the receiver in the $i$ th execution for $x$ being the private input for $\mathbf{S}_{c}^{1}$. That is, for $i=1, \ldots, n$,

$$
\begin{aligned}
A_{i}(x) & =\operatorname{output}_{S}\left(S_{c}^{1}\left(x_{i}\right), R^{*}\left(V_{1}, \ldots, V_{i-1}\right)\right) ; \\
V_{i}(x) & =\operatorname{view}_{R^{*}}\left(S_{c}^{1}\left(x_{i}\right), R^{*}\left(V_{1}, \ldots, V_{i-1}\right)\right) .
\end{aligned}
$$

Let $A^{\prime \prime}\left(\Gamma_{1}^{\prime}\right)=\left(A_{1}\left(\Gamma_{1}^{\prime}\right), \ldots, A_{n}\left(\Gamma_{1}^{\prime}\right)\right)$ represent the combined first-phase private outputs of the $n$ senders and $V^{\prime \prime}\left(\Gamma_{1}^{\prime}\right)=\left(V_{1}\left(\Gamma_{1}^{\prime}\right), \ldots, V_{n}\left(\Gamma_{1}^{\prime}\right)\right)$ represent the view of $R^{*}$ when interacting with these $n$ senders before interactive hashing is done. From now on, we simplify notation by making $A^{\prime \prime}=A^{\prime \prime}\left(\Gamma_{1}^{\prime}\right)$ and $V^{\prime \prime}=V^{\prime \prime}\left(\Gamma_{1}^{\prime}\right)$.

Similar to (4) and as in the proof of Lemma 6.19, we obtain

$$
\mathrm{CP}^{1 / 2}\left(A^{\prime \prime} \mid V^{\prime \prime}\right) \leq \sqrt{2^{-\gamma_{1} \cdot(k-1)}} .
$$

And by a Markov bound, we know that with probability greater than $1-2^{-n}$ over $v^{\prime \prime} \leftarrow V^{\prime \prime}$,

$$
\mathrm{CP}\left(\left.A^{\prime \prime}\right|_{V^{\prime \prime}=v^{\prime \prime}}\right) \leq 2^{-\gamma_{1}(k-1)} \cdot 2^{2 n} \leq 2^{-\alpha_{1}-(1 / 24) \delta k n+3 n} \leq 2^{-\left(\alpha_{1}+n\right)},
$$

with the last inequality following from $k \geq 100 / \delta$.

Consider $v^{\prime \prime} \in V^{\prime \prime}$ such that $(6)$ holds. Let $Q=\left(H_{1}, H_{1}\left(A^{\prime \prime}\right)\right)$, where $H_{1}$ is an independent random hash from $\mathcal{H}_{1}$. Because $H_{1}$ is independent, $\left.Q\right|_{V^{\prime \prime}=v^{\prime \prime}}=$ $\left(H_{1}, H_{1}\left(\left.A^{\prime \prime}\right|_{V^{\prime \prime}=v^{\prime \prime}}\right)\right)$, and we can apply the Leftover Hash Lemma, Lemma 6.8, to obtain that $\left.Q\right|_{V^{\prime \prime}=v^{\prime \prime}}$, the input to the interactive hashing protocol, is $2^{-\Omega(n)}$-close to uniform.

Next, let $A^{\prime}=$ output $_{\mathbf{S}}\left(\mathbf{S}^{1}\left(\Gamma_{1}^{\prime}\right), R^{*}\right)$ denote the private output of $\mathbf{S}$ in the first phase, which in turn is equal to the output of $S_{\mathrm{IH}}$ in the interactive hashing protocol, so equivalently $A^{\prime}=\operatorname{output}_{S_{\mathrm{IH}}}\left(S_{\mathrm{IH}}(Q), R^{*}\right)$. Similarly, let $V^{\prime}=\operatorname{view}_{R^{*}}\left(S_{c}^{1}\left(\Gamma_{1}\right), R^{*}\right)$ denote the view of the adversarial receiver $R^{*}$ in the first phase, and let $V_{\mathrm{IH}}=$ view $_{R_{\mathrm{IH}}^{*}}\left(S_{\mathrm{IH}}(Q), R_{\mathrm{IH}}^{*}\right)$ denote the view of receiver $R^{*}$ during the interactive hashing execution only. Observe that $V^{\prime}=\left(V^{\prime \prime}, V_{\mathrm{IH}}\right)$, recalling that $V^{\prime \prime}$ is the view of $R^{*}$ when interacting with these $n$ senders, before interactive hashing is done. 
Because $\left.Q\right|_{V^{\prime \prime}=v^{\prime \prime}}$, the input to interactive hashing, is $2^{-\Omega(n)}$-close to uniform, we know that $\left(\left.A^{\prime}\right|_{V^{\prime \prime}=v^{\prime \prime}},\left.V_{\mathrm{IH}}\right|_{V^{\prime \prime}=v^{\prime \prime}}\right)$ is $2^{-\Omega(n)}$-close to $\left(U_{n},\left.V_{\mathrm{IH}}\right|_{V^{\prime \prime}=v^{\prime \prime}}\right)$, as guaranteed by the hiding property of interactive hashing (see Definition 3.1). So S's private output $\left.A^{\prime}\right|_{V^{\prime \prime}=v^{\prime \prime}}$ is hidden from $R^{*}$ for any $v^{\prime \prime} \in V^{\prime \prime}$ satisfying (6). Finally, note that (6) is satisfied for all but a $2^{-n}$ fraction of $v^{\prime \prime} \leftarrow V^{\prime \prime}$, so it follows that $\left(A^{\prime}, V^{\prime}\right)$ is $2^{-\Omega(n)}$-close to $\left(U_{n}, V^{\prime}\right)$, as required.

Property (H.3) implies $\left(\mathrm{H}^{\prime} .3\right)$. Using ideas similar to those in the proof of Lemma 6.19 , we can proceed as above and obtain that Property $\left(\mathrm{H}^{\prime} .3\right)$ holds assuming (H.3).

6.3.2. Binding preservation. In the execution of Algorithm 6.17, we obtained $\ell$ intermediate commitment schemes $\left[\left(S_{j}, R_{j}\right)\right]_{1 \leq j \leq \ell}$, and one final commitment scheme $(S, R)$. Our goal is to prove that the final scheme $(S, R)$ satisfies the 1-out-of-2 binding property of Definition 5.4. To achieve our goal, we inductively show that the expected number of openings a sender can produce in the intermediate schemes is bounded by some constant, namely, 32. (This is captured by Lemma 6.22 below.) Then in the final step, for scheme $(S, R)$, we show how to shrink this expectation to a value that is very close to 1 , thereby proving that scheme $(S, R)$ satisfies the 1-out-of-2 binding property. (This in turn is captured by Lemma 6.24.)

In the definition of the computational 1-out-of-2 binding property (Definition 5.4), we stipulated that the adversarial sender in the second phase can be computationally unbounded, whereas the adversarial sender in the first phase must be probabilistic polynomial time (PPT). It will be rather messy to work with PPT senders; hence we will abstract away the PPT requirement by showing, in the next section, how to convert any PPT sender violating the 1-out-of-2 binding property in the first phase into a computationally unbounded sender with a special unique binding property. A sender with the unique binding property, intuitively, will not break the (first-phase) binding property of any execution of the initial schemes $\left(S_{0}, R_{0}\right)$ but might still break the binding property of the intermediate schemes $\left(S_{j}, R_{j}\right)$ (or final scheme $(\mathrm{S}, \mathrm{R})$ ). Intuitively, we can restrict our attention to such senders because of the computational 1-out-of-2 binding property of the initial scheme $\left(S_{0}, R_{0}\right)$. Once we have a sender with the unique binding property, the analysis of the amplification steps is entirely information theoretic.

To formally define the unique binding property for senders, we observe that schemes $\left[\left(S_{j}, R_{j}\right)\right]_{1 \leq j \leq \ell}$ and $(\mathrm{S}, \mathrm{R})$ each contain multiple executions of initial scheme $\left(S_{0}, R_{0}\right)$. Hence, when a cheating sender $S^{*}$ interacts with $R_{j}$, it is actually also interacting with the $i$ th execution of $R_{0}$ for each $i=1,2, \ldots$, which we will denote by $R_{0}[i]$. Formally, we define a (computationally unbounded) cheating sender strategy $S^{*}[i]$ that interacts with this single execution of $R_{0}[i]$ (more precisely, the first commit stage $R_{0, c}^{1}[i]$ ) by simulating all of the other messages of $R_{j}$ on its own until the end of the first commit stage of $R_{0}[i]$. Then it enumerates over all choices for the subsequent messages of $R_{j}$ and outputs all of the resulting full transcripts of $S^{*}$ 's interactions with $R_{0}[i]$ (including both phases).

Let $\mathcal{B}_{0}^{t}$ be the binding set guaranteed by Lemma 6.14 for $\left(S_{0}, R_{0}\right)$ with parameter $t$. Then we define the random variable openings $\left(S^{*}[i], R_{0}^{1}[i]\right)\left(\mathcal{B}_{0}^{t}\right)$ as follows, analogously to section 6.2.4:

- $S^{*}[i]$ and $R_{0, c}^{1}[i]$ interact to get first-phase commitment $c^{(1)}$. (According to the definition of $S^{*}$ above, $S^{*}[i]$ accomplishes this by simulating the rest of the interaction of $S^{*}$ and $R_{j}$ on its own.)

- After the interaction, $S^{*}[i]$ outputs a sequence of values $d_{1}^{(1)}, \ldots, d_{\ell}^{(1)}$ and 
corresponding full transcripts $\lambda_{1}, \ldots, \lambda_{\ell}$ of both phases. Recall that $\lambda_{i}=$ $\left(\tau_{i}, \kappa_{i}\right)$, where $\tau_{i}$ and $\kappa_{i}$ are the first-phase and second-phase transcripts, respectively. (According to the definition of $S^{*}$ above, $S^{*}[i]$ accomplishes this by enumerating over all possible continuations of the interaction of $S^{*}$ and $R_{j}$ and outputting all of the resulting transcripts of the interaction with $R_{0}[i]$.)

- We let openings $\left(S^{*}[i], R_{0}^{1}[i]\right)\left(\mathcal{B}_{0}\right)$ be the set of distinct values $d_{i}^{(1)}$ whose opening $\lambda_{i}$ is valid, where by valid we mean that $\lambda_{i}$ begins with prefix $c^{(1)}, \lambda_{i}$ contains a decommitment of $c^{(1)}$ to the value $d_{i}^{(1)}$ with first-phase transcript $\tau_{i} \notin \mathcal{B}_{0}^{t}$, and both $R_{0, r}^{1}$ and $R_{0, r}^{2}$ accept in $\lambda_{i}$.

DEFINITION 6.21 (unique binding property of sender). For an intermediate scheme $\left(S_{j}, R_{j}\right)$ or the final scheme $(\mathrm{S}, \mathrm{R})$ with parameter $t$, a (deterministic) sender $S^{*}$ has the unique $\mathcal{B}_{0}^{t}$-binding property if for all $i$ we have

$$
\left|\operatorname{openings}\left(S^{*}[i], R_{0}^{1}[i]\right)\left(\mathcal{B}_{0}^{t}\right)\right| \leq 1
$$

with probability 1 (over the coins of $S^{*}[i]^{10}$ and $R_{0}^{1}[i]$ ), where $S^{*}[i]$ and openings $\left(S^{*}[i]\right.$, $\left.R_{0}^{1}[i]\right)\left(\mathcal{B}_{0}^{t}\right)$ are defined as above.

The following two lemmas, Lemmas 6.22 and 6.24 , provide us with a way to understand the binding property (in an average-case sense) of ( $\mathbf{S}, \mathbf{R})$, the amplified hiding scheme as presented in Protocol 6.16, in terms of $(S, R)$. We might occasionally drop the superscripts 1 and 2 from the notation if it is clear which phase we are referring to.

LEMma 6.22 (intermediate step binding preservation). For every sufficiently large constant $D \in \mathbb{N}$, the following holds: For all integers $t \in[1, n], j \in[1, \ell]$, $\beta_{1}, \ldots, \beta_{j} \in\{0,1, \ldots, D-1\}$, letting $\left(S_{j}, R_{j}\right)$ be the intermediate commitment schemes obtained in the execution of Algorithm 6.17 with parameters $D, t$, and $\left(\beta_{1}, \ldots, \beta_{j}\right),{ }^{11}$ there exists a binding set $\mathcal{B}_{j}=\mathcal{B}_{j}\left(t, \beta_{1}, \ldots, \beta_{j}\right)$ such that the following two conditions hold:

(B.1) For every deterministic sender $S^{*}$ with the unique $\mathcal{B}_{0}^{t}$-binding property,

$$
\mathrm{E}\left[\left|\operatorname{openings}\left(S^{*}, R_{j}^{1}\right)\left(\mathcal{B}_{j}\right)\right|\right]<32,
$$

where the expectation is taken over the coin tosses of $R_{j}^{1}$.

(B.2) For every $\tau \in \mathcal{B}_{j}$ and for every deterministic sender $S^{*}$,

$$
\mathrm{E}\left[\left|\operatorname{openings}\left(S^{*}, R_{j}^{2}\right)(\tau)\right|\right]<32,
$$

where the expectation is taken over the coin tosses of $R_{j}^{2}$.

Proof. We proceed to prove by induction on $j$. In fact, we will start with a base case of $j=0$; i.e., consider the scheme $\left(S_{0}, R_{0}\right)$ from section 6.2. By Definition 6.21, the scheme $\left(S_{0}, R_{0}\right)$ satisfies condition (B.1), and by Lemma 6.14 , it satisfies condition (B.2).

For the inductive step, we assume $\left(S_{j}, R_{j}\right)$ satisfy both (B.1) and (B.2) and show that so does $\left(S_{j+1}, R_{j+1}\right)$. Note that $\left(S_{j+1}, R_{j+1}\right)$ is obtained by the amplification procedure (Protocol 6.16) that combines $m$ sequential executions of $\left(S_{j}, R_{j}\right)$, i.e.,

\footnotetext{
${ }^{10}$ Note that $S^{*}[i]$ is probabilistic even if $S^{*}$ is deterministic, because it simulates all of the random choices of $R_{j}$ other than those of $R_{0}[i]$.

${ }^{11}$ Note that $\left(S_{j}, R_{j}\right)$ does not depend on $\beta_{j+1}, \ldots, \beta_{\ell+1}$.
} 
$\left(S_{j+1}, R_{j+1}\right)=\operatorname{Amplify}\left(S_{j}, R_{j}\right)$. Hence, for convenience of notation we will denote $\left(S_{j}, R_{j}\right)$ and $\left(S_{j+1}, R_{j+1}\right)$ as $(S, R)$ and $(\mathbf{S}, \mathbf{R})$, respectively. The $i$ th execution of $(S, R)$ in $(\mathbf{S}, \mathbf{R})$ is denoted as $(S[i], R[i])$, which is not to be confused with the subscript indexing notation of $\left(S_{j}, R_{j}\right)$.

Also, throughout this proof, the value of $m$ will be fixed to $D$, although we will keep writing $m$. Let $\mathcal{B}=\mathcal{B}_{j}$ be the binding set for $(S, R)$. We define our new binding set $\mathcal{B}^{\prime}=\mathcal{B}_{j+1}$ for $(\mathbf{S}, \mathbf{R})$ in terms of $\mathcal{B}$ and $\beta=\beta_{j+1}$ as follows:

$$
\mathcal{B}^{\prime}=\left\{\left(\tau_{1}, \ldots, \tau_{m}\right): \exists j_{1}, \ldots, j_{\beta+1} \text { such that } \tau_{j_{1}}, \ldots, \tau_{j_{\beta+1}} \in \mathcal{B}\right\} .
$$

That is, a transcript $\tau^{\prime}=\left(\tau_{1}, \ldots, \tau_{m}\right) \in \mathcal{B}^{\prime}$ if and only if at least $\beta+1$ of the $\tau_{j}$ 's are in $\mathcal{B}$. So, $\tau^{\prime} \notin \mathcal{B}^{\prime}$ if and only if at least $m-\beta$ of the $\tau_{j}$ 's are not in $\mathcal{B}$.

Property (B.1). Consider a deterministic $S^{*}$ with the unique $\mathcal{B}_{0}^{t}$-binding property interacting with $\mathbf{R}^{1}$. The random coins of $\mathbf{R}^{1}$ can be broken up into independent random coins of $R^{1}[1], \ldots, R^{1}[m]$ and $R_{\mathrm{IH}}^{1}$, the receiver in the interactive hashing.

Recall that the $m$ executions of $(S, R)$ in $(\mathbf{S}, \mathbf{R})$ are sequential. We want to focus on the interaction of $S^{*}$ with (the commit phase of) $R^{1}[i]$. To do so, for each possible setting of the coin tosses $r_{1}, \ldots, r_{i-1}$ for $R^{1}[1], \ldots, R^{1}[i-1]$, define a sender strategy $S^{*}[[i]]\left(r_{1}, \ldots, r_{i-1}\right)$ that interacts with $R^{1}[i]$, as follows: $S^{*}[[i]]\left(r_{1}, \ldots, r_{i-1}\right)$ simulates $S^{*}$ using fixed coins $r_{j}$ for all the previous $R^{1}[j]$ 's (for all $j<i$ ) and after the interaction with $R^{1}[i], S^{*}[[i]]$ outputs all the valid openings that occur in some continuation of $S^{*}$ 's interaction with $R[i]$ (by enumerating over all coins of the future $R[j]$ 's, $j>i$, the coins of $R_{\mathrm{IH}}^{1}$, and the coins of $\left.\mathbf{R}^{2}\right)$. Observe that $S^{*}[[i]]\left(r_{1}, \ldots, r_{i-1}\right)$ inherits the unique $\mathcal{B}_{0}^{t}$-binding property from $S^{*}$.

Let $X_{i}\left(r_{1}, \ldots, r_{i}\right)=\left|\operatorname{openings}\left(S^{*}[i]\left(r_{1}, \ldots, r_{i-1}\right), R^{1}[i]\left(r_{i}\right)\right)(\mathcal{B})\right| ;$ this counts the number of valid decommitments in the $i$ th execution, when the sender uses simulated coins $r_{1}, \ldots, r_{i-1}$ and $R^{1}[i]$ uses coins $r_{i}$. Let $U=\left(U_{1}, \ldots, U_{m}\right)$, where $U_{i}$ denotes the uniform random variable on coins $r_{i}$ for $R[i]$; note that these are independent because the honest receiver tosses independent coins for each execution. We now consider the random variables $X_{i}(U)=X_{i}\left(U_{1}, \ldots, U_{i}\right)$.

By our induction hypothesis applied to $S^{*}[[i]]\left(r_{1}, \ldots, r_{i-1}\right)$, for all fixed $\left(r_{1}, \ldots\right.$, $\left.r_{i-1}\right)$, we have

$$
\underset{U}{\mathrm{E}}\left[X_{i}(U) \mid U_{1}=r_{1}, \ldots, U_{i-1}=r_{i-1}\right]=\underset{U_{i}}{\mathrm{E}}\left[X_{i}\left(r_{1}, \ldots, r_{i-1}, U_{i}\right)\right]<32 .
$$

Because the previous $X_{j}(U)$ 's for $j<i$ depend only on $U_{1}, \ldots, U_{j}$, we have that the expected value of $X_{i}$ is less than 32 even given any previous values of $X_{j}$ 's. That is, $\mathrm{E}\left[\left.X_{i}\right|_{X_{1}=x_{1}, \ldots, X_{i-1}=x_{i-1}}\right]<32$ for every $\left(x_{1}, \ldots, x_{i-1}\right) \in \operatorname{Supp}\left(X_{1}, \ldots, X_{i-1}\right)$. The following claim allows us to bound the expectation of the product of these random variables.

ClaIm 6.23. Let $Y_{1}, \ldots, Y_{\ell}$ be nonnegative real-valued random variables such that for all $i=1,2, \ldots, \ell$, we have $\mathrm{E}\left[\left.Y_{i}\right|_{Y_{1}=y_{1}, \ldots, Y_{i-1}=y_{i-1}}\right]<\alpha_{i} \in \mathbb{R}^{+}$, for every $\left(y_{1}, \ldots, y_{i-1}\right) \in \operatorname{Supp}\left(Y_{1}, \ldots, Y_{i-1}\right)$. Then,

$$
\mathrm{E}\left[\prod_{i=1}^{\ell} Y_{i}\right]<\prod_{i=1}^{\ell} \alpha_{i} .
$$


Proof of Claim 6.23. Note that

$$
\begin{aligned}
\mathrm{E}\left[Y_{1} \cdots Y_{\ell}\right] & =\underset{\left(y_{1}, \ldots, y_{\ell-1}\right) \stackrel{\mathrm{R}}{(}\left(Y_{1}, \ldots, Y_{\ell-1}\right)}{\mathrm{E}}\left[y_{1} \cdots y_{\ell-1} \cdot \mathrm{E}\left[\left.Y_{\ell}\right|_{Y_{1}=y_{1}, \ldots, Y_{\ell-1}=y_{\ell-1}}\right]\right] \\
& <\mathrm{E}\left[Y_{1} \cdots Y_{\ell-1} \cdot \alpha_{\ell}\right] \\
& =\alpha_{\ell} \cdot \mathrm{E}\left[Y_{1} \cdots Y_{\ell-1}\right]
\end{aligned}
$$

and the claim follows by induction on $\ell$.

As noted above, it is always the case that $\mathrm{E}\left[X_{i}\right]<32$, regardless of the instantiation of previous $X_{j}$ 's for $j<i$. Note that Claim 6.23 also applies to computing the expectation of $\prod_{i \in J} X_{i}$ for any subset $J \subset[m]$, since any subset of the $X_{i}$ 's (preserving the right order) satisfies the condition of the claim.

Once the $m$ commitments $R^{1}[i]$ are complete, we can define a random variable $A=A(U)$ that denotes the set of values $a=\left(a_{1}, \ldots, a_{m}\right)$ 's for which the sender $S^{*}$ produces a valid opening with respect to $\mathcal{B}^{\prime}$ in some continuation of the protocol when receiver $R^{1}[i]$ uses coin tosses $U_{i}$. By the definition of $\mathcal{B}^{\prime}$, this means that $a=\left(a_{1}, \ldots, a_{m}\right)$ is valid only if at least $m-\beta$ of those are $a_{i}$ 's corresponding to decommitments that are not in $\mathcal{B}$. For those $a_{i}$ 's corresponding to decommitments that are in $\mathcal{B}$, the number of possible values that $a_{i}$ can take on is $X_{i}(U)$. And for those $a_{i}$ 's corresponding to decommitments that are not in $\mathcal{B}$, we can bound the number of possible values that $a_{i}$ can take on by $2^{k}$ (since $a_{i}$ is a $k$-bit string).

$$
\begin{array}{rlr}
\underset{U}{\mathrm{E}}[|A(U)|] & \leq \underset{U}{\mathrm{E}}\left[\sum_{J \subseteq[m],|J| \geq m-\beta} \prod_{i \in J} X_{i}(U) \prod_{i \notin J} 2^{k}\right] & \\
& =\sum_{J \subseteq[m],|J| \geq m-\beta} \underset{U}{\mathrm{E}}\left[\prod_{i \in J} X_{i}(U) \prod_{i \notin J} 2^{k}\right] & \\
& <\sum_{J \subseteq[m],|J| \geq m-\beta} \prod_{i \in J} 32 \cdot \prod_{i \notin J} 2^{k} \quad \quad \text { (by Claim 6.23) } \\
& \leq 2^{m} \cdot 32^{m-\beta} \cdot\left(2^{k}\right)^{\beta} & \\
& \leq 2^{(\beta+1)(k-1)+6 m-k+1}=2^{\alpha_{1}-(k-6 m-4)} . &
\end{array}
$$

Define random variable $T_{1}=T_{1}(U)=\left\{\left(h_{1}, h_{1}(a)\right): a \in A, h_{1} \in \mathcal{H}_{1}\right\}$. Since $\mathrm{E}[|A|] \leq 2^{\alpha_{1}-(k-6 m-4)}$ and the range of $h_{1} \in \mathcal{H}_{1}$ is of length $\alpha_{1}$, the expected density of $T_{1}$ satisfies $\mathrm{E}\left[\mu\left(T_{1}\right)\right] \leq \mathrm{E}[|A|] \cdot 2^{-\alpha_{1}} \leq 2^{-(k-6 m-4)}$, where the expectations are taken over the coin tosses $U=\left(U_{1}, \ldots, U_{m}\right)$. Note that $T_{1}$ is independent of the coins of $R_{\mathrm{IH}}^{1}$ in the first-phase interactive hashing (though not independent of the coins of $\left.\mathbf{R}^{1}\right)$.

Finally, we have

$$
\underset{\text { coins } \mathbf{R}^{1}}{\mathrm{E}}\left[\mid \text { openings }\left(S^{*}, \mathbf{R}^{1}\right)\left(\mathcal{B}^{\prime}\right) \mid\right] \leq \underset{T_{1}, \text { coins } R_{\mathrm{IH}}^{1}}{\mathrm{E}}\left[\left|\left\{d^{(1)}: C^{(1)}\left(d^{(1)}\right) \in T_{1}\right\}\right|\right],
$$

where, in the second expectation, $C^{(1)}=\operatorname{output}\left(S^{*}, R_{\mathrm{IH}}^{1}\right)$. By Lemma 6.10,

$$
\underset{T_{1}, \text { coins } R_{\mathrm{IH}}^{1}}{\mathrm{E}}\left[\left|\left\{d^{(1)}: C^{(1)}\left(d^{(1)}\right) \in T_{1}\right\}\right|\right]<24+2^{k^{\prime}+1} \cdot \mathrm{E}\left[\mu\left(T_{1}\right)\right]<32,
$$

with the last inequality following from $k^{\prime}=k-8 m-8$. 
Property (B.2). We use the same approach as above, except this time, we consider all deterministic $S^{*}$, as opposed to only those with the unique binding property. Also, we need to fix a binding transcript $\tau=\left(\tau_{1}, \ldots, \tau_{m}\right) \in \mathcal{B}^{\prime}$. Let $J$ be the set of indices such that $\tau_{i} \in \mathcal{B}$.

Analogously to the proof of Property (B.1), we define $S^{*}[[[i]]]\left(r_{1}, \ldots, r_{i-1}\right)$ for every possible setting $r_{1}, \ldots, r_{i-1}$ of coin tosses for receivers $R^{2}[1], \ldots, R^{2}[i-1]$, and we set $X_{i}\left(r_{1}, \ldots, r_{i}\right)=\left|\operatorname{openings}\left(S^{*}[[[i]]]\left(r_{1}, \ldots, r_{i-1}\right), R^{2}[i]\left(r_{i}\right)\right)\left(\tau_{i}\right)\right|$. By our induction hypothesis, for all $i \in J$, we have

$$
\mathrm{E}\left[\left.X_{i}\right|_{X_{1}=x_{1}, \ldots, X_{i-1}=x_{i-1}}\right]<32
$$

for any $\left(x_{1}, \ldots, x_{i-1}\right) \in \operatorname{Supp}\left(X_{1}, \ldots, X_{i-1}\right)$, where we define the random variables $X_{i}=X_{i}\left(U_{1}, \ldots, U_{i}\right)$ and $U=\left(U_{1}, \ldots, U_{m}\right)$ with $U_{i}$ being uniform over the coin tosses $r_{i}$ for $R^{2}[i]$.

Let random variable $B=B(U)$ denote the set of values $b=\left(b_{1}, \ldots, b_{m}\right)$ for which the sender $S^{*}$ produces a valid opening in some continuation of the protocol. Noting that $X_{i}$ can be as large as $2^{k}$ for indices $i \notin J$, we have

$$
\begin{array}{rlrl}
\mathrm{E}[|B|] & \leq \underset{U}{\mathrm{E}}\left[\prod_{i \in J} X_{i} \prod_{i \notin J} 2^{k}\right] & & \\
& <\prod_{i \in J} 32 \cdot \prod_{i \notin J} 2^{k} & & \text { (by Claim } 6.23) \\
& \leq 32^{\beta+1} \cdot\left(2^{k}\right)^{m-\beta-1} & & \text { (because } \left.32<2^{k}\right) \\
& \leq 2^{(m-\beta)(k-1)-(k-6 m)} & & \text { (because } m>5) \\
& =2^{\alpha_{2}-(k-6 m-3)} . &
\end{array}
$$

Let random variable $T_{2}=T_{2}(U)=\left\{\left(h_{2}, h_{2}(b)\right): b \in B, h_{2} \in \mathcal{H}_{2}\right\}$. Since $\mathrm{E}[|B|] \leq$ $2^{\alpha_{2}-(k-6 m-3)}$ and the range of $h_{2} \in \mathcal{H}_{2}$ is $\alpha_{2}$, the expected density of $T_{2}$ satisfies $\mathrm{E}\left[\mu\left(T_{2}\right)\right] \leq \mathrm{E}[|B|] \cdot 2^{-\alpha_{2}} \leq 2^{-(k-6 m-3)}$, where the expectations are taken over $U=$ $\left(U_{1}, \ldots, \bar{U}_{m}\right)$. Note that $\bar{T}_{2}$ is independent of the coins of $R_{\mathrm{IH}}^{2}$ in the second-phase interactive hashing (though not independent of the coins of $\mathbf{R}^{2}$ ). Finally, we have

$$
\underset{\text { coins } \mathbf{R}^{2}}{\mathrm{E}}\left[\left|\operatorname{openings}\left(S^{*}, \mathbf{R}^{2}\right)\left(\tau^{\prime}\right)\right|\right] \leq \underset{T_{2}, \operatorname{coins} R_{\mathrm{IH}}^{2}}{\mathrm{E}}\left[\left|\left\{d^{(2)}: C^{(2)}\left(d^{(2)}\right) \in T_{2}\right\}\right|\right],
$$

where, in the second expectation, $C^{(2)}=\operatorname{openings}\left(S^{*}\left(T_{2}\right), R_{\mathrm{IH}}\right)$. By Lemma 6.10,

$$
\underset{\operatorname{coins} R_{\mathrm{IH}}^{2}, T_{2}}{\mathrm{E}}\left[\left|\left\{d^{(2)}: C^{(2)}\left(d^{(2)}\right) \in T_{2}\right\}\right|\right]<24+2^{k^{\prime}+1} \cdot \mathrm{E}\left[\mu\left(T_{2}\right)\right]<32,
$$

with the last inequality following from $k^{\prime}=k-8 m-8$.

LEMMA 6.24 (final step binding preservation). For every sufficiently large constant $D \in \mathbb{N}$, the following holds: For all integers $t \in[1, n], \beta_{1}, \ldots, \beta_{\ell} \in\{0,1, \ldots, D-$ $1\}$, and $\beta_{\ell+1} \in[0, n]$, letting $(\mathrm{S}, \mathrm{R})$ be the final output of Algorithm 6.17 with parameters $D, t$, and $\left(\beta_{1}, \ldots, \beta_{\ell+1}\right)$, there exists a binding set $\mathcal{B}^{\prime}$ such that the following two conditions hold:

(B.1) For every deterministic sender $S^{*}$ with the unique $\mathcal{B}_{0}^{t}$-binding property, with probability $1-2^{-\Omega(n)}$ over the coins of $\mathrm{R}^{1}$,

$$
\mid \text { openings }\left(S^{*}, \mathrm{R}^{1}\right)\left(\mathcal{B}^{\prime}\right) \mid \leq 1 \text {. }
$$


(B.2) For every $\tau \in \mathcal{B}^{\prime}$ and for every deterministic sender $S^{*}$, with probability $1-2^{-\Omega(n)}$ over the coins of $\mathrm{R}^{2}$,

$$
\mid \text { openings }\left(S^{*}, \mathrm{R}^{2}\right)(\tau) \mid \leq 1 \text {. }
$$

Proof. From Lemma 6.22 , we have scheme $\left(S_{\ell}, R_{\ell}\right)$ with an associated binding set $\mathcal{B}=\mathcal{B}_{\ell}$ satisfying both conditions (B.1) and (B.2) in Lemma 6.22. Scheme (S,R)= Amplify $\left(S_{\ell}, R_{\ell}\right)$, and hence we will need to show that the amplification boosts the binding by making sure both $\mid$ openings $\left(S^{*}, \mathrm{R}^{1}\right)\left(\mathcal{B}^{\prime}\right) \mid \leq 1$ and $\mid$ openings $\left(S^{*}, \mathrm{R}^{2}\right)(\tau) \mid \leq$ 1 with probability $1-2^{-\Omega(n)}$.

Throughout this proof, the value of $m$ will be fixed to $n$ (as in step 3 of Algorithm 6.17), although we will keep writing $m$. We define our new binding set $\mathcal{B}^{\prime}$ for $(\mathrm{S}, \mathrm{R})$ in terms of $\mathcal{B}$ and $\beta=\beta_{\ell+1}$ as follows:

$$
\mathcal{B}^{\prime}=\left\{\left(\tau_{1}, \ldots, \tau_{m}\right): \exists j_{1}, \ldots, j_{\beta+1} \text { such that } \tau_{j_{1}}, \ldots, \tau_{j_{\beta+1}} \in \mathcal{B}\right\} .
$$

That is, a transcript $\tau^{\prime}=\left(\tau_{1}, \ldots, \tau_{m}\right) \in \mathcal{B}^{\prime}$ if and only if at least $\beta+1$ of the $\tau_{j}$ 's are in $\mathcal{B}$. Thus, $\tau^{\prime} \notin \mathcal{B}^{\prime}$ if and only if at least $m-\beta$ of the $\tau_{j}$ 's are not in $\mathcal{B}$.

Property (B.1). Using the same analysis and notation as in the proof of Lemma 6.22 , we have that

$$
\underset{U}{\mathrm{E}}[|A|] \leq 2^{m} \cdot 32^{m-\beta} \cdot\left(2^{k}\right)^{\beta} \leq 2^{\beta k+6 m},
$$

where $A$ is the random variable denoting the set of values $a=\left(a_{1}, \ldots, a_{m}\right)$ for which the sender $S^{*}$ produces a valid opening with respect to $\mathcal{B}^{\prime}$ in some continuation of the protocol and $U=\left(U_{1}, \ldots, U_{m}\right)$ denotes coin tosses for $R^{1}[1], \ldots, R^{1}[m]$.

Since $\delta=\Omega(1)$ and $k=k_{\ell} \geq \log n$, observe that $\alpha_{1}=\left\lfloor\left(\beta+\frac{1}{3} \delta n\right) k\right\rfloor=\beta k+\omega(n)$ for large enough values of $n$. Let random variable $T_{1}=\left\{\left(h_{1}, h_{1}(a)\right): h_{1} \in \mathcal{H}_{1}, a \in\right.$ $A\})$. Since the range of $h_{1} \in \mathcal{H}_{1}$ is $\{0,1\}^{\alpha_{1}}$, the density of $\Gamma_{1}$ satisfies

$$
\underset{U}{\mathrm{E}}\left[\mu\left(T_{1}\right)\right] \leq \mathrm{E}[|A|] \cdot 2^{-\alpha_{1}}<2^{\beta k+6 m} \cdot 2^{-(\beta k+\omega(n))}=2^{-\omega(n)},
$$

since $m=n$. Thus, with probability at least $1-2^{-n}$ over the coin tosses $U$ of $R^{1}[1], \ldots, R^{1}[m]$, we have that

$$
\mu\left(T_{1}\right) \leq 2^{-\omega(n)} \cdot 2^{n} \leq 2^{-2 n} .
$$

By Lemma 3.7, we can conclude that for such a $T_{1}\left(\right.$ with $\left.\mu\left(T_{1}\right) \leq 2^{-2 n}\right)$,

$$
\underset{\text { coins } R_{\mathrm{IH}}^{1}}{\operatorname{Pr}}\left[\left|\left\{d^{(1)}: C^{(1)}\left(d^{(1)}\right) \in T_{1}\right\}\right|>1\right] \leq \operatorname{poly}(n) \cdot\left(2^{-2 n} \cdot 2^{k^{\prime}}\right)^{1 / 2}=2^{-\Omega(n)} .
$$

Finally, we have

$$
\begin{aligned}
& \underset{\text { coins } \mathbf{R}^{1}}{\operatorname{Pr}}\left[\left|\operatorname{openings}\left(S^{*}, \mathbf{R}^{1}\right)\right|>1\right] \\
& \leq \underset{\text { coins } R_{1}^{1}, \cdots, R_{m}^{1}}{\operatorname{Pr}}\left[\mu\left(T_{1}\right)>2^{-2 n}\right] \\
& +\operatorname{Pr}_{\text {coins } R_{\mathrm{IH}}^{1}}\left[\left|\left\{d^{(1)}: C^{(1)}\left(d^{(1)}\right) \in T_{1}\right\}\right|>1 \mid \mu\left(T_{1}\right) \leq 2^{-2 n}\right] \\
& =2^{-\Omega(n)} \text {. }
\end{aligned}
$$


Property (B.2). Fix any $\tau^{\prime} \in \mathcal{B}^{\prime}$. Again, we use the same analysis and notation as in the proof of Lemma 6.22 to get

$$
\underset{U}{\mathrm{E}}[|B|] \leq 32^{\beta+1} \cdot\left(2^{k}\right)^{m-\beta-1} \leq 2^{(m-\beta) k+5 m},
$$

where $B$ is the random variable denoting the set of values $b=\left(b_{1}, \ldots, b_{m}\right)$ for which the sender $S^{*}$ produces a valid opening in some continuation of the protocol and $U=\left(U_{1}, \ldots, U_{m}\right)$ denotes coin tosses for $R^{2}[1], \ldots, R^{2}[m]$.

Since $\delta=\Omega(1)$ and $k \geq \log n$, observe that $\alpha_{2}=\left\lfloor\left(n-\beta+\frac{1}{3} \delta n\right) k\right\rfloor=(n-\beta) k+$ $\omega(n)$ for large enough values of $n$. Let random variable $T_{2}=\left\{\left(h_{2}, h_{2}(b)\right): h_{2} \in\right.$ $\left.\mathcal{H}_{2}, b \in B\right\}$. Since the range of $h_{2} \in \mathcal{H}_{2}$ is $\{0,1\}^{\alpha_{2}}$, the density of $T_{2}$ satisfies

$$
\underset{U}{\mathrm{E}}\left[\mu\left(T_{2}\right)\right] \leq \mathrm{E}[|B|] \cdot 2^{-\alpha_{2}}<2^{(m-\beta) k+5 m} \cdot 2^{-((n-\beta) k+\omega(n))}=2^{-\omega(n)},
$$

since $m=n$. Thus, with probability at least $1-2^{-n}$ over the coin tosses $U$ of $R^{2}[1], \ldots, R^{2}[m]$, we have that

$$
\mu\left(T_{2}\right) \leq 2^{-\omega(n)} \cdot 2^{n} \leq 2^{-2 n}
$$

By Lemma 3.7, we can conclude that for such a $T_{2}\left(\right.$ with $\left.\mu\left(T_{2}\right) \leq 2^{-2 n}\right)$,

$$
\underset{\text { coins } R_{\mathrm{IH}}^{2}}{\operatorname{Pr}}\left[\left|\left\{d^{(2)}: C^{(2)}\left(d^{(2)}\right) \in T_{2}\right\}\right|>1\right]=2^{-\Omega(n)} .
$$

Finally, we have

$$
\begin{aligned}
& \operatorname{Pr}_{\text {coins } \mathbf{R}^{2}}\left[\mid \text { openings }\left(S^{*}, \mathbf{R}^{2}\right)\left(\tau^{\prime}\right) \mid>1\right] \\
& \leq \underset{\text { coins } R_{1}^{2}, \cdots, R_{n}^{2}}{\operatorname{Pr}}\left[\mu\left(T_{2}\right)>2^{-2 n}\right] \\
& +\underset{\text { coins } R_{\mathrm{IH}}^{2}}{\operatorname{Pr}}\left[\left|\left\{d^{(2)}: C^{(2)}\left(d^{(2)}\right) \in T_{2}\right\}\right| \mid \mu\left(T_{2}\right) \leq 2^{-2 n}\right] \\
& =2^{-\Omega(n)} .
\end{aligned}
$$

6.4. A collection of 1-out-of-2-binding commitments. In this section, we prove Theorem 6.1, which is restated below.

Restatement of Theorem 6.1. Given a one-way function $f:\{0,1\}^{n} \rightarrow$ $\{0,1\}^{n}$, we can construct in time polynomial in $n$ a collection of $m=\operatorname{poly}(n)$ publiccoin 2-phase commitment schemes $\mathcal{C O} \mathcal{M}=\left\{\mathrm{Com}_{1}, \ldots, \mathrm{Com}_{m}\right\}$ with message lengths $\left(k_{1}, k_{2}\right)=(n, n)$, such that

- there exists an index $i \in\{1,2, \ldots, m\}$ such that scheme $\mathrm{Com}_{i}$ is statistically hiding, and

- for every index $i \in\{1,2, \ldots, m\}$, scheme $\mathrm{Com}_{i}$ is computationally 1-out-of-2 binding.

6.4.1. Proof of Theorem 6.1. To obtain the desired collection of 2-phase commitment schemes, we apply Algorithm 6.17 to the weakly hiding scheme $\left(S_{0}, R_{0}\right)$, which can be constructed based on any one-way function $f:\{0,1\}^{n} \rightarrow\{0,1\}^{n}$. More precisely, we obtain a collection of commitments by enumerating over all the polynomially many choices of the integers $t \in\{\lceil 2 \log n\rceil, 2, \ldots, n-\lceil 2 \log n\rceil\}, \beta_{1}, \ldots, \beta_{\ell} \in$ $\{0,1, \ldots, D-1\}$, and $\beta_{\ell+1} \in\{0,1, \ldots, n\}$. Note that the number of choices is 
$n \cdot D^{\ell} \cdot(n+1)=\operatorname{poly}(n)$, as $D=O(1)$ and $\ell=\log n$. By Lemma 6.18 , the resulting commitment schemes $\mathrm{Com}_{1}, \ldots, \mathrm{Com}_{m}$ all run in polynomial time. The hiding and binding properties of these schemes are given by Lemmas 6.25 and 6.26 , which together establish Theorem 6.1.

Lemma 6.25. For every sufficiently large constant $D \in N$, the following holds: Let $f:\{0,1\}^{n} \rightarrow\{0,1\}^{n}$ be any function (not necessarily one-way) such that $\left|f^{-1}(y)\right| \in$ $\left[2 n^{2}, 2^{n} / n^{2}\right]$ for every $y \in\{0,1\}^{n}$. Then there exist integers $t \in\{\lceil 2 \log n\rceil, 2, \ldots, n-$ $\lceil 2 \log n\rceil\}, \beta_{1}, \ldots, \beta_{\ell} \in\{0,1, \ldots, D-1\}$, and $\beta_{\ell+1} \in\{0,1, \ldots, n\}$ such that the 2 phase commitment scheme $(\mathrm{S}, \mathrm{R})$ produced by Algorithm 6.17 with parameters $D, t$, and $\left(\beta_{1}, \ldots, \beta_{\ell+1}\right)$ is statistically hiding in the sense of Definition 5.3.

As we observed earlier with Theorem 5.6, the condition on the preimage sizes of $f$ can be easily made to hold by padding the input of $f$. Specifically, given any function $f:\{0,1\}^{n} \rightarrow\{0,1\}^{n}$, the function $f^{\prime}(x, y, z)=(f(x), y)$ with $|x|=|y|=|z|$ has input length $n^{\prime}=3 n$, has all preimage sizes between $2^{n^{\prime} / 3}$ and $2^{2 n^{\prime} / 3}$, and is one-way if $f$ is.

Proof. We prove by induction on $j=0, \ldots, \ell$ that there exist $t, \beta_{1}, \ldots, \beta_{j}$ such that $\left(S_{j}, R_{j}\right)$ is $\delta_{j}$-hiding in $\mathrm{CP}^{1 / 2}$ measure for $\delta_{j}=\min \left\{2^{j} / n, 1 / D\right\}$. The base case of $j=0$ is simply Lemma 6.13 . The induction step is provided by the intermediate step hiding amplification lemma, Lemma 6.19. Specifically, if by induction we choose $t, \beta_{1}, \ldots, \beta_{j-1}$ such that $\left(S_{j-1}, R_{j-1}\right)$ is $\delta_{j-1}$-hiding, the lemma tells us that there exists $\beta_{j}$ such that $\left(S_{j}, R_{j}\right)$ is $\delta^{\prime}$-hiding for $\delta^{\prime}=\min \left\{2 \delta_{j-1}, 1 / D\right\}=\delta_{j}$.

Finally, we apply the final step hiding amplification lemma, Lemma 6.20 , to obtain a $\beta_{\ell+1}$ such that $(S, R)$ is statistically hiding. The lemma applies because $\delta_{\ell}=\min \left\{2^{\ell} / n, 1 / D\right\}=\Omega(1)$ (recall that $\ell=\lceil\log n\rceil$ and $D=O(1)$ ), and $k_{\ell}=$ $(16 D) \cdot \ell-(8 D+8) \cdot \ell>100 / \delta_{\ell}$ for sufficiently large $n$.

Lemma 6.26. For every sufficiently large constant $D$, the following holds: If $f$ : $\{0,1\}^{n} \rightarrow\{0,1\}^{n}$ is one-way, then for all integers $t \in\{\lceil 2 \log n\rceil, 2, \ldots, n-\lceil 2 \log n\rceil\}$, $\beta_{1}, \ldots, \beta_{\ell} \in\{0,1, \ldots, D-1\}$, and $\beta_{\ell+1} \in\{0,1, \ldots, n\}$, the 2-phase commitment scheme $(\mathrm{S}, \mathrm{R})$ produced by Algorithm 6.17 with parameters $D$, $t$, and $\left(\beta_{1}, \ldots, \beta_{\ell+1}\right)$ is computationally 1-out-of-2 binding in the sense of Definition 5.4. (Here the function $f$ on which the scheme is based needs to be hard to invert.)

Proof. Let $\mathcal{B}^{\prime}$ be the binding set given by Lemma 6.24. That lemma establishes that the 2-phase commitment scheme $(\mathrm{S}, \mathrm{R})$ produced by Algorithm 6.17 satisfies the second condition of Definition 5.4 with respect to $\mathcal{B}^{\prime}$. In addition, it also satisfies the first condition for all $S^{*}$ with the unique binding property. Stated formally, for every deterministic (and computationally unbounded) $S^{*}$ with the unique $\mathcal{B}_{0}^{t}$-binding property,

$$
\operatorname{Pr}\left[\mid \text { openings }\left(S^{*}, \mathrm{R}^{1}\right)\left(\mathcal{B}^{\prime}\right) \mid \leq 1\right]=1-2^{-\Omega(n)},
$$

where the probability is taken over the coins of $\mathrm{R}^{1}$.

Thus, it suffices to prove is that any PPT $S^{*}$ breaking the first condition of Definition 5.4 (with respect to $\mathcal{B}^{\prime}$ ) with probability $\varepsilon$ will either (i) yield a $P P T \hat{S}$ that violates the computational 1-out-of-2 binding property of $\left(S_{0}, R_{0}\right)$ with probability at least $\varepsilon^{O(1)} / \operatorname{poly}(n)$, or (ii) yield a computationally unbounded $\hat{S}$ that has the unique $\mathcal{B}_{0}^{t}$-binding property and violates $(7)$.

From now on, let $\varepsilon$ be the probability that $S^{*}$ breaks the second condition of Definition 5.4 with respect to scheme $(\mathrm{S}, \mathrm{R})$. This probability is taken over the coin tosses of both the receiver R and the cheating sender $S^{*}$. We will write $S^{*}(r)$ to denote 
$S^{*}$ with its coin tosses fixed to $r$. By our definition of $(\mathrm{S}, \mathrm{R})$, it contains polynomially many executions of $\left(S_{0}, R_{0}\right)$. Let $N=n \cdot D^{\ell}$ denote the number of such executions.

Let $\mathbf{z}$ denote a transcript of $\left(S^{*}, \mathrm{R}\right)$. Contained in $\mathbf{z}$ is also a first-phase commitment $z[i]$ for the $i$ th execution of $R_{0}$, denoted $R_{0}^{1}[i]$ (for all $i=1,2, \ldots, N$ ). Let $\hat{z}[i]$ be the partial transcript of $\mathbf{z}$ up to and including the first commit stage of $R_{0}[i]$. Note that $z[i]$ is a suffix of $\hat{z}[i]$, and $\hat{z}[i]$ is a prefix of $\mathbf{z}$.

For all indices $i \in[N]$, partial transcripts $\hat{z}[i]$ ending with the first commit stage of $R_{0}[i]$ and $d \in\{0,1\}^{k_{0}}$, and coin tosses $r$ for $S^{*}$, define

$p_{i, \hat{z}[i], d, r}=\operatorname{Pr}_{\mathbf{z} \leftarrow\left(S^{*}(r), \mathrm{R}\right)}[\mathbf{z}$ contains a valid opening of $z[i]$ to value $d \mid \mathbf{z}$ begins with $\hat{z}[i]]$,

where the probability is taken over the coin tosses of $\mathrm{R}$ (which includes coins of the subsequent executions $R_{0}[j], j>i$, as well as coins for many executions of $R_{\mathrm{IH}}$ ). As usual by a valid opening, we mean that $S^{*}$ sends a full transcript $\lambda[i]=(\tau[i], \kappa[i])$ of both phases of its interaction with $R_{0}[i]$ such that $\lambda[i]$ begins with $z[i], \lambda[i]$ contains a decommitment of $z[i]$ to value $d$ with a first-phase transcript $\tau[i] \notin \mathcal{B}_{0}^{t}$, and both $R_{0}^{1}[i]$ and $R_{0}^{2}[i]$ accept in $\lambda[i]$.

Let $K=2^{k_{0}}$, where $k_{0}$ is the message length in $\left(S_{0}, R_{0}\right)$. We have two cases to consider.

Case 1. There exists an $i \in[N]$ such that, with probability at least $\varepsilon / 4 N K$ over $\hat{z}[i]$ and $r$, there exists $d \neq d^{\prime}$ with both $p_{i, \hat{z}[i], d, r}, p_{i, \hat{z}[i], d^{\prime}, r}>\varepsilon / 4 N K$.

In this case, we violate the computational 1-out-of-2 binding property of $\left(S_{0}, R_{0}\right)$ by considering the following sender $\hat{S}$ interacting with $R_{0}[i]$.

1. Select a random $i \leftarrow[N]$ and coin tosses $r$ for $S^{*}$.

2. Run $S^{*}(r)$ with $\mathrm{R}$, simulating all of the messages of $\mathrm{R}$ internally except for those of $R_{0}[i]$. Halting after the first commit stage of $R_{0}[i]$, we obtain a partial transcript $\hat{z}[i]$. From $\hat{z}[i]$, we get $z[i]$, the first-phase commitment of $R_{0}[i]$.

3. Record the current state $\psi$ of $S^{*}(r)$ and R.

4. Continue the execution of $S^{*}(r)$ with $\mathrm{R}$ from $\psi$ twice using independent randomness for $\mathrm{R}$.

5. Examine the transcripts to look for valid decommitments to two different values $d, d^{\prime}$.

Because our goal is to violate the computational 1-out-of-2 binding property of $\left(S_{0}, R_{0}\right)$, we succeed in the above algorithm if $d \neq d^{\prime}$ and decommitments produced are valid. We calculate our success probability as follows: We guess the correct index $i \in$ $[N]$ with probability $1 / N$. Given that we guess the correct $i$, we get the desired $\hat{z}[i]$ and $r$ with probability at least $\varepsilon / 4 N K$. Now, when we do two independent continuations of $\hat{z}[i]$ we arrive at two different decommitted values with probability greater than $(\varepsilon / 4 N K)^{2}$. Consequently, we violate the computational 1-out-of-2 binding property of $\left(S_{0}, R_{0}\right)$ (i.e., win the game in condition 2 of Definition 5.4) with probability greater than

$$
\frac{1}{N} \cdot \frac{\varepsilon}{4 N K} \cdot\left(\frac{\varepsilon}{4 N K}\right)^{2}=\left(\frac{\varepsilon}{n}\right)^{O(1)},
$$

since $K=2^{k_{0}}=2^{O(\log n)}=\operatorname{poly}(n)$ and $N=n \cdot D^{\ell}=n \cdot O(1)^{O(\log n)}=\operatorname{poly}(n)$. This forces $\varepsilon$ to be a negligible function.

Case 2. For all $i \in[N]$, it holds that with probability greater than $1-\varepsilon / 4 N K$ over $\hat{z}[i]$ and $r$, there is at most one $d$ such that $p_{i, \hat{z}[i], d, r}>\varepsilon / 4 N K$. 
Define $d^{*}(\hat{z}[i], r)$ to be the value of $d$ that maximizes $p_{i, \hat{z}[i], d, r}$. Taking a union bound over other possible values $d^{\prime} \neq d^{*}(\hat{z}[i], r)$, we have that

$$
\begin{aligned}
& \operatorname{Pr}_{r, \mathbf{z} \leftarrow\left(S^{*}(r), \mathrm{R}\right)}\left[\text { in } \mathbf{z}, S^{*}(r) \text { opens some } z[i] \text { to a value other than } d^{*}(\hat{z}[i], r)\right] \\
\leq & \sum_{i=1}^{N}\left(\frac{\varepsilon}{4 N K} \cdot K+\operatorname{Pr}_{\hat{z}[i], r}\left[\text { exists more than one } d \text { such that } p_{i, \hat{z}[i], d, r}>\frac{\varepsilon}{4 N K}\right]\right) \\
< & N \cdot\left(\frac{\varepsilon}{4 N K} \cdot K+\frac{\varepsilon}{4 N K}\right) \\
< & \frac{\varepsilon}{2}
\end{aligned}
$$

Let $\hat{S}(r)$ be the adversary that mimics $S^{*}(r)$ except that it halts and fails if $S^{*}(r)$ attempts to open some $z[i]$ to a value other than $d^{*}(\hat{z}[i], r)$ for some $i \in[N]$ and $\hat{z}[i]$. By the above calculations, the final outcome of $\left(\hat{S}, \mathrm{R}^{1}\right)$ will differ only the original final outcome of $\left(S^{*}, \mathrm{R}^{1}\right)$ with probability at most $\varepsilon / 2$ over $r$ and the coins of $\mathrm{R}^{1}$. In addition, for each $r, \hat{S}(r)$ has the unique $\mathcal{B}_{0}^{t}$-binding property. By $(7)$ above, for every $r$, openings $\left(\hat{S}(r), \mathrm{R}^{1}\right)\left(\mathcal{B}^{\prime}\right) \mid>1$ occurs with at most negligible probability over the coins of $\mathrm{R}^{1}$. Hence, $\mid$ openings $\left(S^{*}(r), \mathrm{R}^{1}\right)\left(\mathcal{B}^{\prime}\right) \mid>1$ occurs with probability at most neg $(n)+\varepsilon / 2$ over $r$ and the coins of $\mathrm{R}^{1}$. We started off assuming that $S^{*}$ breaks property (B.1) of scheme (S, R) with probability at least $\varepsilon$, that is to say $\mid$ openings $\left(S^{*}, \mathrm{R}^{1}\right)\left(\mathcal{B}_{0}^{t}\right) \mid>1$ with probability at least $\varepsilon$. Thus $\varepsilon \leq n \operatorname{neg}(n)+\varepsilon / 2$, which implies that $\varepsilon=\operatorname{neg}(n)$.

7. Standard commitments from 1-out-of-2-binding commitments. In this section we show how to transform any $\left(\begin{array}{l}2 \\ 1\end{array}\right)$-binding 2-phase commitment scheme into a standard computationally binding commitment scheme, which is statistically hiding given that the 2-phase commitment is. Applied to our result from the previous section, this gives a family of commitment schemes that are all computationally binding, and one of which is statistically hiding. In the next section, we show how to convert any such family into a single statistically hiding commitment scheme. We accomplish this using a novel application of a universal one-way hash family, whose existence can be based on any one-way function [7] (see also [29]). Thus, our transformation can be based on any one-way function.

7.1. Overview. We would like to use a 2-phase commitment scheme to construct a (standard) commitment scheme. A naive attempt to design the commitment scheme may go as follows: First, the sender commits to some random string $x$ using the first-phase commit stage. Then, the receiver flips a coin phase $\in\{$ first, second $\}$, if phase $=$ first; then the first-phase commitment is used as the commitment (e.g., the sender sends to the receiver the exclusive-or of its secret with $x$ ). Otherwise ( phase $=$ second $)$, the two parties execute the first-phase reveal stage, and if successful (i.e., the receiver does not reject), they use the second-phase commitment (invoked with the transcript of the first phase as input) as the commitment.

The intuition is that since the commitment is $\left(\begin{array}{l}2 \\ 1\end{array}\right)$-binding, the sender cannot cheat in both phases together, and thus the receiver would catch a cheating sender with probability one half. The problem is, however, that the sender can decide in which commitment he likes to cheat after knowing the value of phase. Hence, the sender can cheat successfully in both cases without violating the $\left(\begin{array}{l}2 \\ 1\end{array}\right)$-binding of the underlying protocol. 
Our additional idea is to use universal one-way hash functions (UOWHFs) in order to force the sender to decide in which phase it is about to cheat before knowing the value of phase. A family of universal one-way hash functions is a relaxation of collision-resistant hash functions that were defined by Naor and Yung [23] and shown to be constructable from any one-way function by Rompel [7]. ${ }^{12}$ A family of universal one-way hash functions is a family of compressing functions such that no efficient adversary can succeed in the following game with nonnegligible probability. The adversary should first announce a value $x$. Then, on a uniformly selected hash function $f$ (given to the adversary after it announces $x$ ), it succeeds if it can find $x^{\prime} \neq x$ such that $f\left(x^{\prime}\right)=f(x)$.

Our implementation is as follows: After the first-phase commit stage, the receiver selects a random (universal one-way) hash function $f$ and the sender sends back $y=f(x)$. The protocol proceeds essentially as the naive protocol above, where any time the first-phase reveal stage is executed in the naive protocol revealing the value $x^{\prime}$ (either in the reveal stage for phase $=$ first or in the commit-stage for phase $=$ second), the receiver also verifies that $f\left(x^{\prime}\right)=y$.

Assuming the hash function $f$ is sufficiently compressing, the string $x$ remains quite unpredictable even though $f(x)$ is sent to $R$ (in the new variant of the protocol). Thus, in the case that phase = first, we can still use the "entropy" remaining in $x$ to hide the sender's secret (assuming it is sufficiently shorter than $|x|-|f(x)|$ ). To show the statistical hiding in the complementary case when phase $=$ second, it is sufficient to note that sending $f(x)$ does not compromise the hiding property of the secondphase commitment. All in all, the protocol is statistically hiding for both choices of phase, and thus it is statistically hiding.

To argue about the binding of the protocol, recall that the 1-out-of-2-binding property informally states that with high probability after the first-phase commit stage, there exists a single value $\widetilde{x}$ that allows the sender to cheat in the secondphase commitment. Now, if the sender sends $y$ such that $f(\widetilde{x})=y$, then in order to cheat in the case phase $=$ first, it will have to open the first-phase commitment to a value $x^{\prime} \neq \widetilde{x}$ such that $f\left(x^{\prime}\right)=y=f(\widetilde{x})$. This would imply the breaking of the universal one-way hash function. On the other hand, if $f(\widetilde{x}) \neq y$, then in the case phase $=$ second the sender is forced to open the first-phase commitment to a value different than $\widetilde{x}$. This guarantees that the sender cannot cheat in the second-phase commitment, and thus in this case our protocol is binding. In conclusion, since $y$ is sent before phase is chosen, we are guaranteed that our protocol is weakly binding (since intuitively there always exists a choice of phase that prevents the sender from cheating). We complete the construction by amplifying the above protocol into a full-fledged statistically hiding commitment scheme using standard techniques.

7.2. The transformation. We present the transformation algorithm using an arbitrary family of functions $\mathcal{F}$ and will require only that $\mathcal{F}$ to be a universal one-way hash family when we want to prove the hiding and binding security properties.

Algorithm 7.1. The transformation, denoted as 2-to-1-Transform.

Input: security parameter $1^{n}$, 2-phase commitment scheme $(\mathrm{S}, \mathrm{R})$ with message lengths $\left(k_{1}, k_{2}\right)=(n, 1)$, and a family of functions $\mathcal{F}=\bigcup_{n} \mathcal{F}_{n}=\left\{f:\{0,1\}^{n} \rightarrow\right.$ $\left.\{0,1\}^{m}\right\}$.

Output: Commitment scheme $(\mathbb{S}, \mathbb{R})$ as described by Protocol 7.2.

\footnotetext{
${ }^{12} \mathrm{~A}$ version of Rompel's result [7] that holds also for uniform adversaries was recently written by Katz and Koo [29], who also added missing details and fixed some errors.
} 
Hence, we write the commitment scheme obtained as $(\mathbb{S}, \mathbb{R})=2$-to-1-Transform $((\mathrm{S}$, $\mathrm{R}), \mathcal{F})$.

Protocol 7.2. Standard commitment scheme $(\mathbb{S}, \mathbb{R})$ from 2-phase commitment scheme $(\mathrm{S}, \mathrm{R})$.

Security parameter: $1^{n}$, given as common input to both $\mathbb{S}$ and $\mathbb{R}$.

Sender's private input: Bit $b \in\{0,1\}$.

Commit stage:

1. $\mathbb{S}$ selects a uniform $\sigma \leftarrow\{0,1\}^{n}$.

2. $\mathbb{S}$ and $\mathbb{R}$ engage in $\left(\mathrm{S}_{c}^{1}(\sigma), \mathrm{R}_{c}^{1}\right)\left(1^{n}\right)$, with $\mathbb{S}$ acting as $\mathrm{S}_{c}^{1}$ and $\mathbb{R}$ acting as $\mathrm{R}_{c}^{1}$. Let $c^{(1)}$ be the common output of $\mathrm{S}_{c}^{1}$ and $\mathrm{R}_{c}^{1}$ after the interaction.

3. $\mathbb{R}$ chooses $f \leftarrow \mathcal{F}_{n}$ and sends it to $\mathbb{S}$.

4. $\mathbb{S}$ sends $y=f(\sigma)$ to $\mathbb{R}$.

5 . $\mathbb{R}$ flips a random coin, represented by phase $\leftarrow\{1,2\}$, and sends phase to $\mathbb{S}$.

If phase $=1$, then proceed as follows:

(a) $\mathbb{S}$ selects a random hash $h \leftarrow \mathcal{H}$, where $\mathcal{H}$ is a family of pairwiseindependent hash functions with domain $\{0,1\}^{n}$ and range $\{0,1\}$, and sends $(h, b \oplus h(\sigma))$ to $\mathbb{R}$.

(b) $\mathbb{S}$ and $\mathbb{R}$ both output $\left(c^{(1)}, f, y\right.$, phase $\left.=1, h, b \oplus h(\sigma)\right)$ as the commitment.

If phase $=2$, then proceed as follows:

(a) $\mathbb{S}$ runs $\mathrm{S}_{r}^{1}$ to obtain the decommitment message $\gamma^{(1)}$ and first-phase transcript $\tau$ corresponding to both $\sigma$ and $c^{(1)}$. $\mathbb{S}$ sends $\left(\sigma, \gamma^{(1)}, \tau\right)$ to $\mathbb{R}$.

(b) $\mathbb{S}$ and $\mathbb{R}$ engage in $\left(\mathrm{S}_{c}^{2}(b), \mathrm{R}_{c}^{2}\right)\left(1^{n}, \tau\right)$, with $\mathbb{S}$ acting as $\mathrm{S}_{c}^{2}$ and $\mathbb{R}$ acting as $\mathrm{R}_{c}^{2}$. Let $c^{(2)}$ be the common output of $\mathrm{S}_{c}^{2}$ and $\mathrm{R}_{c}^{2}$ after the interaction.

Reveal stage:

(c) $\mathbb{S}$ and $\mathbb{R}$ both output $\left(c^{(1)}, f, y\right.$, phase $\left.=2, c^{(2)}\right)$ as the commitment.

To decommit to bit $b$, do the following depending the value of phase.

If phase $=1$, then the following occur:

1. $\mathbb{S}$ sends $(b, \sigma)$ to $\mathbb{R}$.

2. If $y=f(\sigma)$ and the last component of the commitment equals $b \oplus h(\sigma)$, then $\mathbb{R}$ accepts. Otherwise, $\mathbb{R}$ rejects.

If phase $=2$, then the following occur:

1. $\mathbb{S}$ runs $\mathrm{S}_{r}^{2}$ to obtain the decommitment message $\gamma^{(2)}$, and sends $\left(b, \gamma^{(2)}\right)$ to $\mathbb{R}$.

2. If $y=f(\sigma)$ and both $\mathrm{R}_{r}^{1}$ and $\mathrm{R}_{r}^{2}$ accept $\left(c^{(1)}, \sigma, \gamma^{(1)}\right)$ and $\left(c^{(2)}, b, \gamma^{(2)}\right)$ respectively, then $\mathbb{R}$ accepts. Otherwise, $\mathbb{R}$ rejects.

7.3. Analyzing the transformation. The hiding and binding security properties of Protocol 7.2 will rely on properties of $\mathcal{F}$ being a universal one-way hash family.

Our plan for the remainder of this section is as follows: (i) we present the definition of a universal one-way hash family due to Naor and Yung [23]; (ii) we separate the properties of a universal one-way hash family into two parts; and finally, (iii) we prove the hiding and binding properties of Protocol 7.2 based on these two separate properties.

Universal one-way hash family. In order to define a universal one-way hash family, we need to understand what it means for a family of functions to be polynomial-time 
computable.

Definition 7.3. A family of functions $\mathcal{F}=\bigcup_{n} \mathcal{F}_{n}=\left\{f:\{0,1\}^{n} \rightarrow\{0,1\}^{m}\right\}$ is polynomial-time computable if the following hold.

- Every function $f \in \mathcal{F}_{n}$ is described by a bitstring of length $p(n)$ for some polynomial p. By abuse of notation, we also denote this description by $f$ and write $f \stackrel{R}{\leftarrow} \mathcal{F}_{n}$ to mean that it is chosen uniformly at random in $\{0,1\}^{p(n)}$. (A more general definition would allow the description of the function to be selected according to any polynomial-time samplable distribution, even one that requires private coin tosses. However, our stronger "public-coin" definition is achieved by existing constructions and can be useful in applications such as constructing public-coin zero-knowledge arguments.)

- There exists a deterministic polynomial-time algorithm $F$ such that for every $n$ and every $f \in \mathcal{F}_{n}$, given the description of the function $f$ and a string $x \in\{0,1\}^{n}, F$ outputs the value of $f(x)$.

Definition 7.4. A polynomial-time computable family of functions $\mathcal{F}=\bigcup_{n} \mathcal{F}_{n}=$ $\left\{f:\{0,1\}^{n} \rightarrow\{0,1\}^{m}\right\}$ is a universal one-way hash family if $m<n$ and for all PPT A the following is negligible in $n$ :

$$
\operatorname{Pr}\left[(x, \text { state }) \leftarrow A\left(1^{n}\right), f \leftarrow \mathcal{F}_{n}, x^{\prime} \leftarrow A(x, \text { state }, f): x^{\prime} \neq x \bigwedge f\left(x^{\prime}\right)=f(x)\right] .
$$

\section{Remark 7.5.}

- In the above definition, we allow the adversary to transfer additional information, i.e., state, between the selection of $x$ and finding the collision. This state variable does not appear in the definition in Katz and Koo [29], which is otherwise identical to the above. However, any universal one-way hash family $\mathcal{F}$ meeting their weaker definition can be converted into one meeting the above definition by selecting $f \stackrel{\mathrm{R}}{\leftarrow} \mathcal{F}$ and $s \stackrel{\mathrm{R}}{\leftarrow}\{0,1\}^{n}$ and defining $f^{\prime}(x)=f(x \oplus s)$. (Intuitively, the random shift $s$ turns an arbitrary point $x$ selected by the adversary into a uniformly random point out of the adversary's control.) The original definition of Naor and Yung [23] (also used by Rompel [7]) does not involve the adversary before $f$ is chosen at all but rather requires that for all $x \in\{0,1\}^{n}, A(x, f)$ has a low probability of producing a collision (over the choice of $f$ and $A$ 's coin tosses). Their definition is suited for the case of nonuniform security (as the arbitrary $x$ can be viewed as nonuniform advice), in which case it becomes equivalent to ours (since $A$ can also have state hardwired nonuniformly).

- Although it is more natural for the security be parameterized in terms of the output length, namely, $m$, our applications do not require hash functions that are shrinking by more than a polynomial factor. Hence, for this reason, and in part for consistency, we keep $n$ as our security parameter.

- Naor and Yung [23] showed that by starting with a universal one-way hash family that is compressing by only one bit, namely, $m=n-1$, more compression can be achieved, say, $m \leq n / 2$, by iterative application of several hash functions chosen from the family. Moreover, it is easy to verify that the same construction holds also with respect to Definition 7.4. Hence, without loss of generality, we can assume that our universal one-way hash family has the feature that $m \leq n / 2$.

Two properties of a universal one-way hash family. A universal one-way hash family satisfying Definition 7.4 has the following two main properties. 
Large preimages: Most of the preimages have a large size. This follows from the compressing nature of hash functions: the output length $m$ is much shorter than the input length $n$. (Recall that we can get a universal one-way hash family with $m \leq n / 2$.) We formalize this property in Definition 7.6.

Target collision resistance: It is hard to find collisions with a value $x$ announced before the hash function is given. We formalize this property in Definition 7.7

DeFinition 7.6. A family of functions $\mathcal{F}=\bigcup_{n} \mathcal{F}_{n}=\left\{f:\{0,1\}^{n} \rightarrow\{0,1\}^{m}\right\}$ has the large preimages property if for every $f \in \mathcal{F}$ most elements in the range of $f$ have large preimage sizes. Stated precisely, there exist a function $\alpha(n)=\omega(1)$ and a negligible function $\varepsilon$ such that for all values of $n$, the following holds:

$$
\operatorname{Pr}_{x \leftarrow\{0,1\}^{n}}\left[\left|f^{-1}(f(x))\right| \geq n^{\alpha(n)}\right] \geq 1-\varepsilon(n)
$$

for every function $f \in \mathcal{F}_{n}$.

Definition 7.7. A family of functions $\mathcal{F}=\bigcup_{n} \mathcal{F}_{n}=\left\{f:\{0,1\}^{n} \rightarrow\{0,1\}^{m}\right\}$ has the statistical (resp., computational) target collision resistance property if for every $A$ (resp., every PPT) the following is negligible in $n$ :

$$
\operatorname{Pr}\left[(x, \text { state }) \leftarrow A\left(1^{n}\right), f \leftarrow \mathcal{F}_{n}, x^{\prime} \leftarrow A(x, \text { state }, f): x^{\prime} \neq x \bigwedge f\left(x^{\prime}\right)=f(x)\right] .
$$

Remark 7.8. In this paper we are using only families of functions that are computational target collision-resistant. Yet whenever possible we also state the results with respect to families with statistical target collision-resistant, because this generalization has proved useful in subsequent work [39].

Large preimages and target collision-resistance are opposing properties. Specifically, it is impossible for a single family of functions to have large preimages and have statistical target collision resistance. The power of a universal one-way hash family comes from the fact that it has the large preimages property and has computational target collision resistance.

Lemma 7.9. If $\mathcal{F}=\bigcup_{n} \mathcal{F}_{n}=\left\{f:\{0,1\}^{n} \rightarrow\{0,1\}^{m}\right\}$ for $m \leq n / 2$ is a universal one-way hash family, then $\mathcal{F}$ has both the large preimages and the computational target collision-resistance property.

Proof. The computational target collision resistance property follow directly from Definition 7.4. Hence, all we need to show is that the compressing nature of $\mathcal{F}$, when $m \leq n / 2$, implies the large preimages property.

Fix $f \in \mathcal{F}_{n}$ and group the elements with small preimages into a set $S=\{y \in$ $\left.\{0,1\}^{m}:\left|f^{-1}(y)\right|<2^{\frac{3}{4} n-m}\right\}$. Since $m \leq n / 2$, every element $y \notin S$ has a preimage of size $\left|f^{-1}(y)\right| \geq 2^{\frac{3}{4} n-m} \geq 2^{n / 4}=n^{\omega(1)}$. To complete the proof, we bound the probability of landing in $S$, by a union bound over the elements in $S$ (for which there are at most $\left.2^{m}\right)$ :

$$
\begin{aligned}
\operatorname{Pr}_{x \leftarrow\{0,1\}^{n}}[f(x) \in S] & =\operatorname{Pr}\left[\exists y \in S \text { with } f\left(U_{n}\right)=y\right] \\
& <\frac{2^{\frac{3}{4} n-m}}{2^{n}} \cdot 2^{m}=2^{-n / 4}=\operatorname{neg}(n) .
\end{aligned}
$$

Hiding. Having separated the properties of a universal one-way hash family into those having large preimages and those having target collision resistance, we now show that the large preimages property of $\mathcal{F}$ translates to the hiding property of the commitment scheme $(\mathbb{S}, \mathbb{R})=2$-to-1-Transform $((\mathrm{S}, \mathrm{R}), \mathcal{F})$. 
LEMma 7.10. If the family of functions $\mathcal{F}$ has the large preimages property, and the 2-phase commitment scheme $(\mathrm{S}, \mathrm{R})$ is statistically hiding, then scheme $(\mathbb{S}, \mathbb{R})=$ 2-to-1-Transform $((\mathrm{S}, \mathrm{R}), \mathcal{F})$ is statistically hiding.

Proof. We need to show that for any adversarial receiver $R^{*}$ the views of $R^{*}$ in $\left(\mathbb{S}(0), R^{*}\right)$ and $\left(\mathbb{S}(1), R^{*}\right)$ are statistically indistinguishable. (In this proof, we drop the security parametrization of $1^{n}$ because it is clear from context.) We can, without loss of generality, consider only deterministic $R^{*}$ because we can fix the adversary's coin tosses to maximize its distinguishing advantage. In the rest of this proof, we use indistinguishability and hiding to mean those of the statistical variant.

Let $P$ denote the value of phase sent by $R^{*}$, and we break our hiding analysis into cases in which $P=1$ and $P=2$. To formalize this case analysis, we say that random variables $X$ and $Y$ are indistinguishable on event $E$ if for all $D$, $|\operatorname{Pr}[D(X)=1 \wedge E]-\operatorname{Pr}[D(Y)=1 \wedge E]|$ is negligible (in the security parameter $n$ ). We will show that the random variables view $R^{*}\left(\mathbb{S}(0), R^{*}\right)$ and view $R^{*}\left(\mathbb{S}(1), R^{*}\right)$ are indistinguishable on both events $P=1$ and $P=2$, thus allowing us to conclude that the scheme is hiding.

First, we analyze the case when $P=2$. Let the random variables $\Sigma$ and $F$ denote $\mathbb{S}^{\prime}$ s choice of $\sigma$ and the value of $f$ sent by $R^{*}$, respectively. Observe that $P$ is a deterministic function of the random variables $V_{1}=\operatorname{view}_{R^{*}}\left(\mathrm{~S}_{c}^{1}(\Sigma), R^{*}\right)$ and $Y=F(\Sigma)$. In turn, $V_{1}$ and $Y$ are deterministic functions of the first-phase transcript $\mathrm{T}=\operatorname{transcript}\left(\mathrm{S}^{1}(\Sigma), R^{*}\right)$, which includes both the commit and reveal stages. This is because we can compute the view of the receiver from the first-phase transcript and the first-phase transcript also contains the value of $\sigma$, from which we can compute $y=f(\sigma)$. For bit $b \in\{0,1\}$, let random variable $V_{2}(b)=\operatorname{view}_{R^{*}}\left(\mathrm{~S}_{c}^{2}(b), R^{*}\right)(\mathrm{T})$, recalling that $\mathrm{T}=\operatorname{transcript}\left(\mathrm{S}^{1}(\Sigma), R^{*}\right)$. Because $(\mathrm{S}, \mathrm{R})$ is hiding, its second-phase commitment is hiding even given the first-phase transcript: this means that $\left(V_{2}(0), \mathrm{T}\right)$ is indistinguishable from $\left(V_{2}(1), \mathrm{T}\right)$. Since $P$ is a deterministic function of $\mathrm{T}$, random variables $\left(V_{2}(0), \mathrm{T}\right)$ and $\left(V_{2}(1), \mathrm{T}\right)$ are indistinguishable on event $P=2$. Finally, since view $\left.R^{*}\left(\mathbb{S}(b), R^{*}\right)\right|_{P=2}$ is a deterministic function of $\left.\left(V_{2}(b), \mathrm{T}\right)\right|_{P=2}$ for $b \in\{0,1\}$, we have that view $R^{*}\left(\mathbb{S}(0), R^{*}\right)$ and view $R^{*}\left(\mathbb{S}(1), R^{*}\right)$ are indistinguishable on event $P=2$.

Next, we analyze the case when $P=1$. The hiding property of the first phase gives us

$$
\left(V_{1}, \Sigma\right) \approx_{s}\left(V_{1}, U_{n}\right)
$$

where $U_{n}$ represents a uniform random variable over $\{0,1\}^{n}$ and is independent from $V_{1}$ and $\Sigma$. Recall that the random variable $F$ denotes the function $f$ sent by $R^{*}$. Since $F$ is a deterministic function of $V_{1}$, we get

$$
\left(V_{1}, F, F(\Sigma), \Sigma\right) \approx_{s}\left(V_{1}, F, F\left(U_{n}\right), U_{n}\right) .
$$

Now, let the random variable $H$ represent the hash function $h$ selected by $\mathbb{S}$ when phase $=1$. Note that $H$ is independent of $V_{1}, F, \Sigma$, and $U_{n}$, so

$$
\left(V_{1}, F, Y, H, H(\Sigma)\right) \approx_{s}\left(V_{1}, F, F\left(U_{n}\right), H, H\left(U_{n}\right)\right),
$$

recalling that $Y=F(\Sigma)$.

We need to establish that $H\left(U_{n}\right)$ is close to uniform so that we have hiding. The next claim does this for us.

CLAIM 7.11. Suppose the family of functions $\mathcal{F}=\bigcup_{n} \mathcal{F}_{n}$ has the large preimages property. Let the random variable $H$ denote a random hash function from a family 
of pairwise-independent hash functions with domain $\{0,1\}^{n}$ and range $\{0,1\}$, let random variable $U_{n}$ denote a uniform string in $\{0,1\}^{n}$, let random variable $U_{1}^{\prime}$ denote a uniform string in $\{0,1\}$, and let $H, U_{n}$, and $U_{1}^{\prime}$ all be independent. For every $f \in \mathcal{F}_{n}$, $\left(f\left(U_{n}\right), H, H\left(U_{n}\right)\right)$ is indistinguishable from $\left(f\left(U_{n}\right), H, U_{1}^{\prime}\right)$.

Proof of Claim. The large preimages property of $\mathcal{F}$ guarantees that with probability $1-\operatorname{neg}(n)$ over $y \leftarrow f\left(U_{n}\right)$, the min-entropy $\mathrm{H}_{\infty}\left(\left.U_{n}\right|_{f\left(U_{n}\right)=y}\right) \geq \omega(\log n)$. For $y$ satisfying this condition, we apply the Leftover Hash Lemma, Lemma 4.1, to get that $\left(y, H, H\left(\left.U_{n}\right|_{f\left(U_{n}\right)=y}\right)\right)$ is indistinguishable from $\left(y, H, H\left(\left.U_{n}\right|_{f\left(U_{n}\right)=y}\right)\right)$.

Because $H$ and $U_{n}$ are independent from the rest of the random variables (and are independent from each other), Claim 7.11 states that

$$
\left(V_{1}, F, F\left(U_{n}\right), H, H\left(U_{n}\right)\right) \approx_{s}\left(V_{1}, F, F\left(U_{n}\right), H, U_{1}^{\prime}\right)
$$

where $U_{1}^{\prime}$ is an independent random variable representing a uniform random variable over $\{0,1\}$. Combining (8) and (9), we get

$$
\left(V_{1}, F, Y, H, H(\Sigma)\right) \approx_{s}\left(V_{1}, F, F\left(U_{n}\right), H, U_{1}^{\prime}\right)
$$

which leads to

$$
\begin{aligned}
\left(V_{1}, F, Y, H, 0 \oplus H(\Sigma)\right) & \approx_{s}\left(V_{1}, F, F\left(U_{n}\right), H, 0 \oplus U_{1}^{\prime}\right) \\
& \equiv\left(V_{1}, F, F\left(U_{n}\right), H, 1 \oplus U_{1}^{\prime}\right) \\
& \approx_{s}\left(V_{1}, F, Y, H, 1 \oplus H(\Sigma)\right) .
\end{aligned}
$$

Since $P$ is a deterministic function of $V_{1}$ and $Y$, random variables $\left(V_{1}, F, Y, H, 0 \oplus\right.$ $H(\Sigma))$ and $\left(V_{1}, F, Y, H, 1 \oplus H(\Sigma)\right)$ are indistinguishable on event $P=1$. Since view $\left._{R^{*}}\left(\mathbb{S}(b), R^{*}\right)\right|_{P=1}$ is a deterministic function of $\left.\left(V_{1}, F, Y, H, b \oplus H(\Sigma)\right)\right|_{P=1}$ for $b \in\{0,1\}$, we have that view $R^{*}\left(\mathbb{S}(0), R^{*}\right)$ and view $R^{*}\left(\mathbb{S}(1), R^{*}\right)$ are indistinguishable on event $P=1$.

Binding. We show that the target collision resistance property of $\mathcal{F}$ translates to the binding property of the commitment scheme $(\mathbb{S}, \mathbb{R})=2$-to-1-Transform $((\mathrm{S}, \mathrm{R}), \mathcal{F})$ obtained from the 2-to-1-Transform. Because we will be able to show only that $(\mathbb{S}, \mathbb{R})$ is binding with probability close to $1 / 2$, we first define what it means to for a scheme to be binding with probability $\delta$ for some $\delta \in[0,1]$.

Definition 7.12. Commitment scheme $(S, R)$ is statistically (resp., computationally) $\delta(n)$-binding if, for every $S^{*}$ (resp., every PPT) and every large enough value of $n$, sender $S^{*}$ succeeds in the following game with probability at most $\delta(n)$ :

On security parameter $1^{n}, S^{*}$ interacts with $R$ in the commit stage obtaining commitment $c$. Then $S^{*}$ outputs pairs $\left(0, d_{0}\right)$ and $\left(1, d_{1}\right)$ and succeeds if in the reveal stage $R\left(0, d_{0}, c\right)=R\left(1, d_{1}, c\right)=$ accept.

The standard notion of binding as given in Definition 2.4 corresponds to being computationally $1 / p(n)$-binding for every polynomial $p$.

LEMMA 7.13. If the family of functions $\mathcal{F}$ is statistically (resp., computationally) target collision-resistant, and the 2-phase commitment scheme $(\mathrm{S}, \mathrm{R})$ is statistically (resp., computationally) $\left(\begin{array}{l}2 \\ 1\end{array}\right)$-binding, then the scheme $(\mathbb{S}, \mathbb{R})=2$-to-1-Transform $((\mathrm{S}, \mathrm{R})$, $\mathcal{F})$ is statistically (resp., computationally) $(1 / 2+1 / p(n))$-binding for every polynomial $p$ and sufficiently large $n$.

Proof. We will focus on the case of computational binding. The statistical case will follow from the fact that the proof is "black-box." Specifically, our proof will (implicitly) give efficient reductions $M_{1}, M_{2}$ such that given any sender strategy 
$S^{*}$ that breaks the $(1 / 2+1 / p(n))$-binding property of $(\mathbb{S}, \mathbb{R})$ as oracle, either $M_{1}^{S^{*}}$ will break the target collision resistance property of $\mathcal{F}$ with nonnegligible probability or $M_{2}^{S^{*}}$ will break the $\left(\begin{array}{l}2 \\ 1\end{array}\right)$-binding property of $(\mathrm{S}, \mathrm{R})$. If both $\mathcal{F}$ and $(\mathrm{S}, \mathrm{R})$ have statistical (resp., computational) security, then this is impossible for every strategy (resp., every PPT strategy) $S^{*}$, and we deduce that $(\mathbb{S}, \mathbb{R})$ must be statistically (resp., computationally) $(1 / 2+1 / p(n))$-binding.

Unless stated otherwise, we take probabilities over the entire interaction between $S^{*}$ and $\mathbb{R}$ in both the commit and reveal stages. We say that $S^{*}$ succeeds if it is able to produce decommitments to two different messages for commitment $\Upsilon$ in the reveal phase (recall that the reveal stage is noninteractive). We want to prove that $\operatorname{Pr}\left[S^{*}\right.$ succeeds $] \leq 1 / 2+1 / p(n)$. We will do this by breaking the probability space into events $E_{1}, \ldots, E_{5}$ corresponding to the various cases in the intuitive proof outline given in section 7.1. We will show that $\operatorname{Pr}\left[\bigvee_{i} E_{i}\right]=1, \operatorname{Pr}\left[E_{1}\right]=1 / 2$, and $\operatorname{Pr}\left[S^{*}\right.$ succeeds $\wedge$ $\left.E_{i}\right] \leq 1 / 4 p(n)$ for $i=2, \ldots, 5$, and this will suffice to prove the lemma.

The first event, $E_{1}$, will depend on the random variables $C=\operatorname{view}_{S^{*}}\left(S^{*}, \mathrm{R}_{c}^{1}\right)$, representing $S^{*}$ 's view of the first-phase commit (this determines the entire state of the interaction $\left(S^{*}, R\right)$, since by Definition 5.1 the honest receiver maintains no private state after the commit phase other than the commitment string); $Y$, denoting the hash value sent by $S^{*}$ after the first-phase commit; $P$, representing the value of phase; and $F$, representing the choice of the function $f \stackrel{\mathrm{R}}{\leftarrow} \mathcal{F}$. We would also like to consider whether or not $Y$ equals $f\left(\Sigma^{*}\right)$, where $\Sigma^{*}$ intuitively represents the value to which $C$ is a commitment, i.e., the "unique" value that will enable $S^{*}$ to break the binding property of the second phase. However, since the commitment scheme may be only computationally binding, $\Sigma^{*}$ is not defined information-theoretically. Thus, we define it as the most likely value to which $S^{*}$ will open the first-phase commitment (with a transcript not in $\mathcal{B})$. Formally, for each first-phase commit transcript $c \in \operatorname{Supp}(C)$, we define

$$
p[\sigma \mid c]=\operatorname{Pr}\left[\begin{array}{c}
\left(S^{*}, R\right) \text { outputs an accepting full transcript } \lambda=(\tau, \kappa) \\
\text { such that } \tau \notin \mathcal{B} \text { and } \tau \text { contains an opening to } \sigma,
\end{array} \mid C=c\right],
$$

where the probability is over the random coins of $S^{*}$ and $R$, and we say full transcript $\lambda$ is accepting if both $R_{r}^{1}$ and $R_{r}^{2}$ accept in $\lambda$. With this measure, we define $\sigma^{*}[c]=$ $\operatorname{argmax}_{\sigma} p[\sigma \mid c]$, breaking ties arbitrarily (say, by choosing the lexicographic smallest $\sigma)$. Then we define the random variable $\Sigma^{*}=\sigma^{*}[C]$.

The intuition described in section 7.1 suggests a case analysis based on whether or not $Y=F\left(\Sigma^{*}\right)$. According to that intuition, the scheme will be binding if $Y=F\left(\Sigma^{*}\right)$ and $P=1$ (by target collision-resistance of $\mathcal{F}$ ) or if $Y \neq F\left(\Sigma^{*}\right)$ and $P=2$ (by the 1out-of-2 binding property), and these events happen with probability $1 / 2$ (because $P$ is randomly chosen after $\Sigma^{*}, F$, and $Y$ are determined). This intuition can be turned directly into a proof in the case that $\mathcal{F}$ has nonuniform target collision-resistance, since the value of $\Sigma^{*}$ (which is determined before $F$ ) can be hardwired into the adversary breaking $\mathcal{F}$. However, to prove our result for uniform adversaries as claimed, we need to ensure that $\Sigma^{*}=\sigma^{*}[C]$ can be efficiently computed (before being given $F$, as per Definition 7.7). We observe that this is the case if $p\left[\Sigma^{*} \mid C\right]>1 / 4 p(n)$, because then if we simulate a continuation of the execution of $\left(S^{*}, R\right)$ starting after $C$, we have a nonnegligible probability of $\Sigma^{*}$ being revealed. On the other hand, the case that $p\left[\Sigma^{*} \mid C\right] \leq 1 / 4 p(n)$ turns out to be analyzable similarly to the case that $Y \neq F\left(\Sigma^{*}\right)$; in both cases we simply use the fact that $S^{*}$ is unlikely to produce a successful opening to $\Sigma^{*}$. 
With the above in mind, we begin by analyzing the event in which we do not expect the scheme to be binding.

Claim 7.14. For the event

$$
E_{1}=\left\{\begin{array}{r}
{\left[\left(Y=F\left(\Sigma^{*}\right)\right) \wedge\left(p\left[\Sigma^{*} \mid C\right]>1 / 4 p(n)\right)\right] \wedge[P=2]} \\
\vee\left[\left(Y \neq F\left(\Sigma^{*}\right)\right) \vee\left(p\left[\Sigma^{*} \mid C\right] \leq 1 / 4 p(n)\right)\right] \wedge[P=1]
\end{array}\right\},
$$

we have $\operatorname{Pr}\left[E_{1}\right]=1 / 2$.

Proof of Claim. $P$ is chosen randomly in $\{1,2\}$ after $C, \Sigma^{*}, F$, and $Y$ are determined.

Now we want to show that the scheme is binding on the complement of $E_{1}$. First we handle the case that $P=1$.

Claim 7.15. For the event

$$
E_{2}=\left\{\left[Y=F\left(\Sigma^{*}\right)\right] \wedge\left[p\left[\Sigma^{*} \mid C\right]>1 / 4 p(n)\right] \wedge[P=1]\right\},
$$

we have $\operatorname{Pr}\left[S^{*}\right.$ succeeds $\left.\wedge E_{2}\right] \leq 1 / 4 p(n)$.

Proof of Claim. Suppose for contradiction that $\operatorname{Pr}\left[S^{*}\right.$ succeeds $\left.\wedge E_{2}\right]>1 / 4 p(n)$; we will show that we can break the target collision-resistance property of $\mathcal{F}$ with nonnegligible probability. In order to do so, we need to output an element $x$ before seeing the hash function, and then given a random function $f \stackrel{R}{\leftarrow} \mathcal{F}$, we need to output $x^{\prime} \neq x$ such that $f(x)=f\left(x^{\prime}\right)$. We do this as follows. First we simulate the interaction between $S^{*}$ and $R$ up to the end of the first-phase commitment and record $c$ as the sender's view so far. Then we continue the interaction from $c$ to the end and set $x$ to be the value of $\sigma$ sent by $S^{*}$ in the protocol (if no valid value is given, we set $x$ to some default value). (In case phase $=1$ and $S^{*}$ produces two values for $\sigma$ in breaking the scheme, choose one of the two at random.) Now we output $x$, store state $=c$, and receive a random hash function $f \stackrel{R}{\leftarrow} \mathcal{F}$. We now rerun the interaction between $S^{*}$ and $R$, starting with the view $(c, f)$, and set $x^{\prime}$ to be the value of $\sigma$ sent by $S^{*}$ in the protocol (again choosing randomly if phase $=1$ and $S^{*}$ produces two values).

To see that this strategy breaks the target collision-resistance property with nonnegligible probability, consider the second completed execution of the interaction between $S^{*}$ and $R$ (the one with the given, random, hash function $f$, which we now denote as a random variable $F)$. By assumption, with probability greater than $1 / 4 p(n)$ in this execution, it holds that $S^{*}$ succeeds, $Y=F\left(\Sigma^{*}\right), p\left[\Sigma^{*} \mid C\right]>1 / 4 p(n)$, and $P=1$. Since $S^{*}$ succeeds and $P=1$, it must be the case that $S^{*}$ produces two successful openings $\Sigma_{1}, \Sigma_{2}$ to the first-phase commit. At least one of these is different from $\Sigma^{*}$, yet both must satisfy $F\left(\Sigma_{i}\right)=Y=F\left(\Sigma^{*}\right)$. With probability at least $1 / 2$, we output $\Sigma_{i} \neq \Sigma^{*}$ as $x^{\prime}$. Now, conditioned on all this, we argue that we had nonnegligible probability (of at least $(1 / 2) \cdot 1 / 4 p(n)$ ) of outputting $\Sigma^{*}$ as $x$ (prior to receiving $F$ ). This follows because $p\left[\Sigma^{*} \mid C\right]>1 / 4 p(n)$. Therefore, we break the target collisionresistance property with probability at least $(1 / 4 p(n)) \cdot(1 / 2) \cdot(1 / 2) \cdot(1 / 4 p(n))$, which is a contradiction.

Now we turn to the complement of $E_{1}$ in case $P=2$, namely, the event

$$
E^{\prime}=\left\{\left[\left(Y \neq F\left(\Sigma^{*}\right)\right) \vee\left(p\left[\Sigma^{*} \mid C\right] \leq 1 / 4 p(n)\right)\right] \wedge[P=2]\right\} .
$$

Since we are now restricted to $P=2$, there is a single first-phase decommitment value produced by $S^{*}$ which we denote by the random variable $\Sigma$.

First we argue that it is almost always the case in $E^{\prime}$ that $\Sigma \neq \Sigma^{*}$ (assuming $S^{*}$ succeeds). 
Claim 7.16. For the event

$$
E_{3}=E^{\prime} \wedge\left(\Sigma=\Sigma^{*}\right),
$$

we have $\operatorname{Pr}\left[S^{*}\right.$ succeeds $\left.\wedge E_{3}\right] \leq 1 / 4 p(n)$.

Proof of Claim. In $E^{\prime}$, either we have $Y \neq F\left(\Sigma^{*}\right)$, in which case $S^{*}$ cannot succeed unless $\Sigma \neq \Sigma^{*}$, or we have $p\left[\Sigma^{*} \mid C\right] \leq(1 / 4(p(n)))$, in which case $S^{*}$ successfully opens to value $\Sigma^{*}$ with probability at most $1 / 4 p(n)$.

So now, instead of $E^{\prime}$, we can focus on the event that $\left\{\left[\Sigma \neq \Sigma^{*}\right] \wedge[P=2]\right\}$. For this, we have two cases, depending on whether the transcript $\mathrm{T}$ of the first-phase commitment (including the reveal) gives a binding second phase or not.

Claim 7.17. For the event

$$
E_{4}=\left\{\left[\Sigma \neq \Sigma^{*}\right] \wedge[P=2] \wedge[\mathrm{T} \in \mathcal{B}]\right\},
$$

we have

$$
\operatorname{Pr}\left[S^{*} \text { succeeds } \wedge E_{4}\right] \leq 1 / 4 p(n) .
$$

Proof of Claim. If $\mathrm{T} \in \mathcal{B}$, then the second-phase commitment is binding. Since $P=2, S^{*}$ can succeed only with negligible probability.

Claim 7.18. For the event

$$
E_{5}=\left\{\left[\Sigma \neq \Sigma^{*}\right] \wedge[P=2] \wedge[\mathrm{T} \notin \mathcal{B}]\right\}
$$

we have

$$
\operatorname{Pr}\left[S^{*} \text { succeeds } \wedge E_{5}\right] \leq 1 / 4 p(n) .
$$

Proof of Claim. Assume for contradiction that $\operatorname{Pr}\left[S^{*}\right.$ succeeds $\left.\wedge E_{5}\right]>1 / 4 p(n)$. By Markov bound, this implies that with probability at least $1 / 8 p(n)$ over $c \stackrel{R}{\leftarrow} C$, it holds that

$$
\operatorname{Pr}\left[S^{*} \text { succeeds } \wedge E_{5} \mid C=c\right]>1 / 8 p(n) .
$$

We will use this to break the $\left(\begin{array}{l}2 \\ 1\end{array}\right)$-binding of $(S, R)$. Similarly to the proof of Claim 7.15, we carry out two executions of $\left(S^{*}, \mathrm{R}\right)$ beginning with the same first-phase commit $c$. Assume that $c$ satisfies (11). Then, with some probability $q[c]$ greater than $1 / 8 p(n)$, the first execution will produce an accepting full transcript whose first phase is not in $\mathcal{B}$, with an opening to some value $\sigma \neq \sigma^{*}=\sigma^{*}[c]$. Note that conditioned on $C=c$, the probability that a random execution produces an accepting full transcript with an opening to some $\sigma^{\prime} \neq \sigma$ must also be at least $q[c]$, since $\sigma$ is no more likely than $\sigma^{*}[c]$. It follows that with probability at least $(1 / 8 p(n)) \cdot q[c] \cdot q[c]=\Omega\left(1 / p(n)^{3}\right)$, we can output two transcripts $\lambda$ and $\widetilde{\lambda}$ such that the following hold:

- both transcripts are accepting and start with $c$,

- the part of the first-phase commitment in both transcripts is not in $\mathcal{B}$,

- the first-phase commitment in $\lambda$ is decommitted to a different value than the one in $\widetilde{\lambda}$.

Namely, we break the $\left(\begin{array}{l}2 \\ 1\end{array}\right)$-binding of $(\mathrm{S}, \mathrm{R})$ with nonnegligible probability.

With the above claims, we complete the proof. By inspection, we have $\operatorname{Pr}\left[\bigvee_{i} E_{i}\right]=$ 1 , and thus

$$
\operatorname{Pr}\left[S^{*} \text { succeeds }\right] \leq \operatorname{Pr}\left[E_{1}\right]+\sum_{i=1}^{4} \operatorname{Pr}\left[S^{*} \text { succeeds } \wedge E_{i}\right] \leq \frac{1}{2}+\frac{1}{p(n)},
$$

as desired. 
Boosting the binding. The commitment scheme $(\mathbb{S}, \mathbb{R})$ from Lemma 7.13 is only $\left(\frac{3}{4}+\operatorname{neg}(n)\right)$-binding. Nonetheless, by the following "folklore" claim, $(\mathbb{S}, \mathbb{R})$ implies a commitment scheme that is neg $(n)$-binding and preserves the same hiding property as the original scheme.

ClaIm 7.19. There exists an efficient procedure that for any function $\delta \geq$ $1 / \operatorname{poly}(n)$ converts a statistically (resp., computationally) $(1-\delta(n))$-binding commitment scheme $(\mathbb{S}, \mathbb{R})$ into a commitment scheme $(S, R)$ that is statistically (resp., computationally) binding. Furthermore, if $(\mathbb{S}, \mathbb{R})$ is statistically (resp., computationally) hiding, so is $(S, R)$.

Proof. The protocol $(S, R)$ is defined as follows: in order to commit to a bit $b$, the two parties run $t=\lceil n / \delta\rceil=\operatorname{poly}(n)$ independent executions of the commit stage of $(\mathbb{S}(b), \mathbb{R})$ one after the other, where $S$ and $R$ act as $\mathbb{S}$ and $\mathbb{R}$, respectively. In the reveal stage, $S$ decommits, via the reveal stage of $(\mathbb{S}, \mathbb{R})$, all the $t$ commitments and $R$ accepts if and only if all the commitments are opened successfully to the same value. The hiding of the above scheme follows by a straightforward hybrid argument. For the binding part, let $S^{*}$ be a PPT trying to break the binding of $(S, R)$. We show that $S^{*}$ breaks the binding of $(S, R)$ only with negligible probability, and since $S^{*}$ was arbitrarily chosen, it follows that $(S, R)$ is computationally binding.

We say that $S^{*}$ breaks the binding of the ith execution of $(\mathbb{S}, \mathbb{R})$ if while trying to break the binding of $(S, R)$ it successfully opens the $i$ th commitment into two different values. Notice that this event depends on several random variables: $C_{<i}$, the coins of $S^{*}$ and the coins of $R$ in the first $i-1$ executions; $C_{i}$, the coins of $R$ in the $i$ th execution; and $C_{>i}$, the coins of $R$ in executions $i+1, \ldots, t$. For settings $\left(c_{<i}, c_{i}\right) \in \operatorname{Supp}\left(C_{<i}, C_{i}\right)$, we define $q_{i}\left(c_{<i}, c_{i}\right)$ to be the probability over $C_{>i}$ that $S^{*}$ breaks the binding of the $i$ th execution conditioned on $\left(C_{<i}, C_{i}\right)=\left(c_{<i}, c_{i}\right)$.

For an arbitrary positive polynomial $p$, define a prefix $c_{<i}$ as bad if $\operatorname{Pr}\left[q_{i}\left(c_{<i}, C_{i}\right)>\right.$ $1 / p(n)]>1-\delta+1 / p(n)$, and otherwise call $c_{<i}$ good. We will now show that $\operatorname{Pr}\left[C_{<i}\right.$ is bad $] \leq 1 / p(n)$. Suppose not. Then we can construct an efficient algorithm $\mathbb{S}^{*}$ that breaks the binding of $(\mathbb{S}, \mathbb{R})$ with probability $1-\delta+1 / 3 p(n)$. In the commit stage, $\mathbb{S}^{*}$ first finds a value $c_{<i}$ for which $\operatorname{Pr}\left[q_{i}\left(c_{<i}, C_{i}\right)>1 / 2 p(n)\right]>1-\delta+1 / 2 p(n)$ and "hardwires" this value into $S^{*}$. Note that the above can be done efficiently and with overwhelming success probability by random sampling, given oracle access to $S^{*} \cdot{ }^{13}$

When interacting with $\mathbb{R}, \mathbb{S}^{*}$ acts as $S^{*}$ does in the $i$ th execution of $\left(S^{*}, R\right)$. With probability at least $1-\delta+1 / 2 p(n)$ over the coins $c_{i}$ of $\mathbb{R}$, we have $q_{i}\left(c_{<i}, c_{i}\right)>1 / 2 p(n)$. If this occurs, then by randomly continuing the simulation of $\left(S^{*}, R\right)$ with $O(n \cdot p(n))$ independent choices of $C_{>i}, \mathbb{S}^{*}$ will be able to break the binding with probability $1-\operatorname{neg}(n)$. Thus, $\mathbb{S}^{*}$ breaks the binding of $(\mathbb{S}, \mathbb{R})$ with probability $1-\delta+1 / 2 p(n)-$ $\operatorname{neg}(n)>1-\delta+1 / 3 p(n)$.

Let $E_{1}$ be the event that for some $i, C_{<i}$ is bad. By the above and a union bound, $\operatorname{Pr}\left[E_{1}\right] \leq t / p(n)$. Let $E_{2}$ be the event that for some $i, q_{i}\left(C_{<i}, C_{i}\right) \leq 1 / p(n)$ but $S^{*}$ breaks the binding of the $i$ th execution. By the definition of $q_{i}$, we have

\footnotetext{
${ }^{13}$ For any given value of $c_{<i}$, we can evaluate, with save but negligible error probability, the value of $\operatorname{Pr}\left[q_{i}\left(c_{<i}, C_{i}\right)>1 / 2 p(n)\right]$ up to an additive error $1 / 4 p(n)$. (In order to do so, we sample $n \cdot p(n)$ uniformly chosen continuations of the commit and reveal stages of $\left(S^{*}, R\right)$, and we compute the fraction of continuations in which $S^{*}$ breaks the binding of the $i$ th commitment). Thus, we can find a value of $c_{<i}$ for which $\operatorname{Pr}\left[q_{i}\left(c_{<i}, C_{i}\right)>1 / 2 p(n)\right]>1-\delta+1 / 2 p(n)$ by sampling $n \cdot p(n)$ values for $c_{<i}$, and for each of these values we evaluate $\operatorname{Pr}\left[q_{i}\left(c_{<i}, C_{i}\right)>1 / 2 p(n)\right]$.
} 
$\operatorname{Pr}\left[E_{2}\right] \leq t / p(n)$. Finally, we have

$$
\begin{aligned}
& \operatorname{Pr}\left[S^{*} \text { breaks the binding } \wedge \neg E_{1} \wedge \neg E_{2}\right] \\
& \leq \operatorname{Pr}\left[\bigwedge_{i=1}^{t}\left[\left(C_{<i} \text { good }\right) \wedge\left(q_{i}\left(C_{<i}, C_{i}\right)>1 / p(n)\right)\right]\right] \\
& =\prod_{i=1}^{t} \operatorname{Pr}\left[\left(C_{<i} \text { good }\right) \wedge\left(q_{i}\left(C_{<i}, C_{i}\right)>1 / p(n)\right) \mid \bigwedge_{j<i}\left[\left(C_{<j} \text { good }\right)\right.\right. \\
& \left.\left.\qquad \wedge\left(q_{j}\left(C_{<j}, C_{j}\right)>1 / p(n)\right)\right]\right] \\
& \leq(1-\delta+1 / p(n))^{t} \\
& =\operatorname{neg}(n)+t / p(n),
\end{aligned}
$$

where the last inequality can be seen by considering any fixed value $C_{<i}=c_{<i}$, which fixes the event on which we are conditioning in the $i$ th factor and whether $C_{<i}$ is good or bad. If $c_{<i}$ is bad, then the probability in the $i$ th factor is 0 . If $c_{<i}$ is good, then the probability (over just $\left.C_{i}\right)$ is at most $(1-\delta+1 / p(n))$ by the definition of good. Taking $p(n)$ to be an arbitrarily large polynomial, we deduce that $S^{*}$ breaks the binding with negligible probability.

Having established the appropriate claims and lemmas, we now state what is achievable from our transformation.

THEOREM 7.20. There exists an efficient procedure (call it 2-to-1-FullTransform) that takes as input a security parameter $1^{n}$, a 2-phase commitment scheme $(\mathrm{S}, \mathrm{R})$ with message lengths $\left(k_{1}, k_{2}\right)=(n, 1)$, and a family of functions $\mathcal{F}=\bigcup_{n} \mathcal{F}_{n}=$ $\left\{f:\{0,1\}^{n} \rightarrow\{0,1\}^{m}\right\}$ and outputs a commitment scheme $(S, R)=2$-to-1-FullTransform $((\mathrm{S}, \mathrm{R}), \mathcal{F})$ satisfying the following properties:

- If $(\mathrm{S}, \mathrm{R})$ is statistically hiding and $\mathcal{F}$ has the large preimages property, then $(S, R)$ is statistically hiding.

- If $(\mathrm{S}, \mathrm{R})$ is statistically (resp., computationally) $\left(\begin{array}{l}2 \\ 1\end{array}\right)$-binding and $\mathcal{F}$ has statistical (resp., computational) target collision-resistance, then $(S, R)$ is statistically (resp., computationally) binding (in the standard sense of binding).

- If $(\mathrm{S}, \mathrm{R})$ is public coin, then $(S, R)$ is also public coin.

Proof. We describe the 2-to-1-FullTransform algorithm, recapping what we have done thus far, as follows.

1. Apply Algorithm 7.1 on $(\mathrm{S}, \mathrm{R})$ and $\mathcal{F}$ to obtain a (standard) commitment scheme $(\mathbb{S}, \mathbb{R})$. Lemmas 7.10 and 7.13 state that for the right properties of both $\left(\mathrm{S}^{\prime}, \mathrm{R}^{\prime}\right)$ and $\mathcal{F}$ (see the first two items in 7.20 above), $(\mathbb{S}, \mathbb{R})$ is hiding and $(1 / 2+\operatorname{neg}(n))$-binding.

2. Next, using Claim 7.19 , boost the binding of $(\mathbb{S}, \mathbb{R})$ to obtain a scheme $(S, R)$ that is neg $(n)$-binding while not affecting the hiding property. Output $(S, R)$ as our desired scheme.

As for the preservation of the public coin property, observe that the messages sent by $\mathbb{R}$ that are specific to the 2 -to-1-Transform are choosing $f \leftarrow \mathcal{F}$ and selecting phase $\leftarrow\{0,1\}$, both of which are public coin operations.

8. Putting it together. Now, we put together everything from the previous sections to establish our main theorem.

Restatement of Theorem 1.1. Given a one-way function $f:\{0,1\}^{n} \rightarrow\{0,1\}^{n}$, we can construct in time polynomial in $n$ a public-coin commitment scheme $(\mathbb{S}, \mathbb{R})$ that 
is statistically hiding and computationally binding.

The statistical hiding property holds regardless of whether or not $f$ is secure (hard to invert). On the other hand, if $f$ is uniformly (resp., nonuniformly) secure, than $(\mathbb{S}, \mathbb{R})$ will be computationally binding with uniform (resp., nonuniform) security.

Proof of Theorem 1.1. We start off by constructing a collection of 2-phase commitment schemes from $f$ using Theorem 6.1. For any polynomial $k(n)$ (which we will choose below) we can construct in time polynomial in $n$ a collection of $m=\operatorname{poly}(n)$ public-coin 2-phase commitment schemes $\mathcal{C O M}=\left\{\mathrm{Com}_{1}, \ldots, \mathrm{Com}_{m}\right\}$ with message lengths $(k(n), 1)$ such that

- there exists an index $i \in\{1,2, \ldots, m\}$ such that scheme $\operatorname{Com}_{i}$ is statistically hiding, and

- for every index $i \in\{1,2, \ldots, m\}$, scheme $\operatorname{Com}_{i}$ is computationally $\left(\begin{array}{l}2 \\ 1\end{array}\right)$-binding. (As remarked after Theorem 6.1, we can obtain 2-phase commitments with message lengths $(k(n), k(n))$ for any polynomial $k$ that we choose. Using only 1 bit of the second-phase message (padding with $k-1$ zeros), we obtain message lengths $(k, 1)$.)

Now in order to apply Theorem 7.20 , from $f$ we use $[7,29]$ to obtain a universal one-way hash family $\mathcal{F}_{n}=\left\{f:\{0,1\}^{k(n)} \rightarrow\{0,1\}^{k(n) / 2}\right\}$ for some polynomial $k$ (which we use to determine the message length for the 2-phase commitment above). ${ }^{14}$ Let the resulting (standard) commitment schemes be $\mathrm{Com}_{i}^{\prime}=$ 2-to-1-FullTransform $\left(\mathrm{Com}_{i}, \mathcal{F}\right)$. By Theorem 7.20 and Lemma 7.9, we know that

- $\mathrm{Com}_{i}^{\prime}$ is statistically hiding if $\mathrm{Com}_{i}$ is statistically hiding,

- $\mathrm{Com}_{i}^{\prime}$ is computationally binding if $\mathrm{Com}_{i}$ is computationally $\left(\begin{array}{l}2 \\ 1\end{array}\right)$-binding, and

- $\mathrm{Com}_{i}^{\prime}$ is public coin if $\mathrm{Com}_{i}$ is public coin.

This means that we now have a collection of public-coin (standard) commitment schemes $\mathcal{C O} \mathcal{M}^{\prime}=\left\{\mathrm{Com}_{1}^{\prime}, \ldots, \operatorname{Com}_{m}^{\prime}\right\}$, where $m=\operatorname{poly}(n)$, such that

- there exists an index $i \in\{1,2, \ldots, m\}$ such that scheme Com $_{i}^{\prime}$ is statistically hiding, and

- for every index $i \in\{1,2, \ldots, m\}$, scheme $\mathrm{Com}_{i}^{\prime}$ is computationally binding (in the standard sense of binding).

We are almost done, except that we are still left with a collection of commitments instead of a single commitment scheme. The following claim states that the latter collection can be converted into the desired commitment scheme.

CLAIM 8.1. There is an efficient procedure that converts a polynomial collection of commitment schemes, at least one of which is statistically hiding and all of which are computationally binding, into a single commitment scheme that is statistically hiding and computationally binding. In addition, if we start off with public-coin schemes, we also end up with a public-coin scheme.

Proof. To commit to a bit $b$, we randomly secret-share $b=b_{1} \oplus \cdots \oplus b_{m}$ and commit to share $b_{i}$ using the $i$ th commitment scheme. Alternatively, the proposition can be deduced from [25, Thm. 5.2].

The main theorem statement is now complete since we now have a single commitment scheme that is statistically hiding and computationally binding, and the only complexity assumption made is the existence of one-way functions.

\footnotetext{
${ }^{14}$ Since we are using here the uniform definition of universal one-way hash family (i.e., where $x$ is sampled by $A$ ), we need to use the theorem of Katz and Koo [29]. In their theorem, however, it is not explicitly defined whether or not the adversary can encode additional information (i.e., state) between the declaration of $x$ and finding the collision (see Remark 7.5). Fortunately, the stronger version of this theorem required by our proof follows readily from their original proof.
} 
We now proceed to the additional properties mentioned. By inspection, we observe that the statistical hiding properties throughout the construction hold regardless of the security of $f$ (see, e.g., Lemma 6.13). As for nonuniform security, we observe that our construction is "fully black-box" in the sense of [40]; in particular, the computational binding property is proven by specifying for every polynomial $p$ a PPT reduction $R$ such that if $\mathbb{S}^{*}$ is any sender strategy (of arbitrary complexity) that breaks the binding property with probability $1 / p(n)$, then $R^{\mathbb{S}^{*}}$ inverts $f$ with nonnegligible probability. In particular, if $\mathbb{S}^{*}$ is a nonuniform PPT algorithm, then we obtain a nonuniform PPT inverter for $f$, which cannot exist if $f$ is nonuniformly secure.

\section{Appendix. Collision probability lemmas.}

We prove the lemmas presented in section 6.2.1.

Restatement of Lemma 6.4. For independent pairs of random variables $\left(X_{1}, Y_{1}\right)$, $\ldots,\left(X_{m}, Y_{m}\right)$,

$$
\mathrm{CP}^{1 / 2}\left(\left(X_{1}, \ldots, X_{m}\right) \mid\left(Y_{1}, \ldots, Y_{m}\right)\right)=\prod_{i=1}^{m} \mathrm{CP}^{1 / 2}\left(X_{i} \mid Y_{i}\right) .
$$

Note that $X_{i}$ and $Y_{i}$ can be correlated; it is required only that the pair $\left(X_{i}, Y_{i}\right)$ be independent from the other tuples.

Proof. Since the $X_{i}$ 's are independent, for all $y_{1}, \ldots, y_{m}$, we have

$$
\mathrm{CP}\left(\left.\left(X_{1}, \ldots, X_{m}\right)\right|_{Y_{1}=y_{1}, \ldots, Y_{m}=y_{m}}\right)=\prod_{i=1}^{m} \mathrm{CP}\left(\left.X_{i}\right|_{Y_{i}=y_{i}}\right) .
$$

This gives us

$$
\begin{array}{ll}
\mathrm{CP}^{1 / 2}\left(\left(X_{1}, \ldots, X_{m}\right) \mid\left(Y_{1}, \ldots, Y_{m}\right)\right) & \\
=\underset{\left(Y_{1}, \ldots, Y_{m}\right)}{\mathrm{E}}\left[\mathrm{CP}^{1 / 2}\left(\left.\left(X_{1}, \ldots, X_{m}\right)\right|_{Y_{1}, \ldots, Y_{m}}\right)\right] & \\
=\underset{\left(Y_{1}, \ldots, Y_{m}\right)}{\mathrm{E}}\left[\prod_{i=1}^{m} \mathrm{CP}^{1 / 2}\left(\left.X_{i}\right|_{Y_{i}}\right)\right] & \text { (by 12) } \\
=\prod_{i=1}^{m} \underset{Y_{i}}{\mathrm{E}}\left[\mathrm{CP}^{1 / 2}\left(\left.X_{i}\right|_{Y_{i}}\right)\right] & \text { (by independence of } \left.Y_{i}^{\prime} \mathrm{s}\right) \\
=\prod_{i=1}^{m} \mathrm{CP}^{1 / 2}\left(X_{i} \mid Y_{i}\right) . &
\end{array}
$$

Restatement of Lemma 6.5. Suppose random variables $\left(X_{1}, Y_{1}\right), \ldots,\left(X_{m}, Y_{m}\right)$ satisfy the following conditions for some values of $\alpha_{1}, \ldots, \alpha_{m} \in \mathbb{R}^{+}$and all $i=$ $1,2, \ldots, m$ :

1. For every $\left(y_{1}, \ldots, y_{i-1}\right) \in \operatorname{Supp}\left(Y_{1}, Y_{2}, \ldots, Y_{i-1}\right)$,

$$
\mathrm{CP}^{1 / 2}\left(\left.X_{i}\right|_{Y_{1}=y_{1}, \ldots, Y_{i-1}=y_{i-1}}\left|Y_{i}\right|_{Y_{1}=y_{1}, \ldots, Y_{i-1}=y_{i-1}}\right) \leq \alpha_{i} .
$$

2. For every $\left(y_{1}, \ldots, y_{i}\right) \in \operatorname{Supp}\left(Y_{1}, Y_{2}, \ldots, Y_{i}\right)$, the $i+1$ random variables $X_{1}, X_{2}, \ldots, X_{i}$, and $Y_{i+1}$ are independent after conditioning on $Y_{1}=y_{1}, \ldots$, $Y_{i}=y_{i}$. 
Then,

$$
\mathrm{CP}^{1 / 2}\left(\left(X_{1}, \ldots, X_{m}\right) \mid\left(Y_{1}, \ldots, Y_{m}\right)\right) \leq \prod_{i=1}^{m} \alpha_{i} .
$$

Proof. By induction, it suffices to prove

$\mathrm{CP}^{1 / 2}\left(\left(X_{1}, \ldots, X_{m}\right) \mid\left(Y_{1}, \ldots, Y_{m}\right)\right) \leq \alpha_{m} \cdot \mathrm{CP}^{1 / 2}\left(\left(X_{1}, \ldots, X_{m-1}\right) \mid\left(Y_{1}, \ldots, Y_{m-1}\right)\right)$,

and then by iteratively expanding $\mathrm{CP}^{1 / 2}\left(\left(X_{1}, \ldots, X_{m-1}\right) \mid\left(Y_{1}, \ldots, Y_{m-1}\right)\right)$ in terms of $\alpha_{j}$ 's, we get our result. To simplify notation, we write $X_{m}^{\prime}=\left.X_{m}\right|_{Y_{1}=y_{1}, \ldots, Y_{m-1}=y_{m-1}}$ and $Y_{m}^{\prime}=\left.Y_{m}\right|_{Y_{1}=y_{1}, \ldots, Y_{m-1}=y_{m-1}}$ when $y_{1}, \ldots, y_{m-1}$ are clear from context. We prove (13) as follows:

$$
\begin{aligned}
& \mathrm{CP}^{1 / 2}\left(\left(X_{1}, \ldots, X_{m}\right) \mid\left(Y_{1}, \ldots, Y_{m}\right)\right) \\
& =\underset{\left(Y_{1}, \ldots, Y_{m}\right)}{\mathrm{E}}\left[\mathrm{CP}^{1 / 2}\left(\left.\left(X_{1}, \ldots, X_{m}\right)\right|_{Y_{1}, \ldots, Y_{m}}\right)\right] \\
& =\underset{\left(Y_{1}, \ldots, Y_{m-1}\right)}{\mathrm{E}}\left[\underset{Y_{m}^{\prime}}{\mathrm{E}}\left[\mathrm{CP}^{1 / 2}\left(\left.\left(X_{1}, \ldots, X_{m}\right)\right|_{Y_{1}, \ldots, Y_{m}^{\prime}}\right)\right]\right] \\
& =\underset{\left(Y_{1}, \ldots, Y_{m-1}\right)}{\mathrm{E}}\left[\underset{Y_{m}^{\prime}}{\mathrm{E}}\left[\mathrm{CP}^{1 / 2}\left(\left.\left(X_{1}, \ldots, X_{m-1}\right)\right|_{Y_{1}, \ldots, Y_{m}^{\prime}}\right) \cdot \mathrm{CP}^{1 / 2}\left(\left.X_{m}\right|_{Y_{1}, \ldots, Y_{m}^{\prime}}\right)\right]\right] \\
& =\underset{\left(Y_{1}, \ldots, Y_{m-1}\right)}{\mathrm{E}}\left[\mathrm{CP}^{1 / 2}\left(\left.\left(X_{1}, \ldots, X_{m-1}\right)\right|_{Y_{1}, \ldots, Y_{m-1}}\right) \cdot \underset{Y_{m}^{\prime}}{\mathrm{E}}\left[\mathrm{CP}^{1 / 2}\left(\left.X_{m}\right|_{Y_{1}, \ldots, Y_{m}^{\prime}}\right)\right]\right] \\
& =\underset{\left(Y_{1}, \ldots, Y_{m-1}\right)}{\mathrm{E}}\left[\mathrm{CP}^{1 / 2}\left(\left.\left(X_{1}, \ldots, X_{m-1}\right)\right|_{Y_{1}, \ldots, Y_{m-1}}\right) \cdot \mathrm{CP}^{1 / 2}\left(X_{m}^{\prime} \mid Y_{m}^{\prime}\right)\right] \\
& \leq \alpha_{m} \cdot \underset{\left(Y_{1}, \ldots, Y_{m-1}\right)}{\mathrm{E}}\left[\mathrm{CP}^{1 / 2}\left(\left.\left(X_{1}, \ldots, X_{m-1}\right)\right|_{Y_{1}, \ldots, Y_{m-1}}\right)\right] \\
& \leq \alpha_{m} \cdot \mathrm{CP}^{1 / 2}\left(\left(X_{1}, \ldots, X_{m-1}\right) \mid\left(Y_{1}, \ldots, Y_{m-1}\right)\right) .
\end{aligned}
$$

Equation (17) follows because $X_{1}, \ldots, X_{m}$ conditioned on $Y_{1}=y_{1}, \ldots, Y_{m}=y_{m}$ are independent. Equation (18) follows because $X_{1}, \ldots, X_{m-1}$ and $Y_{m}$ conditioned on $Y_{1}=y_{1}, \ldots, Y_{m-1}=y_{m-1}$ are independent. Finally, (20) follows from the assumption that for all $\left(y_{1}, \ldots, y_{i-1}\right) \in \operatorname{Supp}\left(Y_{1}, Y_{2}, \ldots, Y_{m-1}\right)$,

$\mathrm{CP}^{1 / 2}\left(X_{m}^{\prime} \mid Y_{m}^{\prime}\right)=\mathrm{CP}^{1 / 2}\left(\left.X_{m}\right|_{Y_{1}=y_{1}, \ldots, Y_{m-1}=y_{m-1}}\left|Y_{m}\right|_{Y_{1}=y_{1}, \ldots, Y_{m-1}=y_{m-1}}\right) \leq \alpha_{m}$.

Restatement of Lemma 6.6. (Randomness Extraction Lemma.) Let $(X, Y)$ be any (possibly correlated) pair of random variables, and let random variable $H$ denote a random hash function from a family of pairwise-independent hash functions $\mathcal{H}$ with range $\{0,1\}^{\alpha}$. Suppose the hash functions from $\mathcal{H}$ are represented by $(q-\alpha)$-bit strings and $\mathrm{CP}^{1 / 2}(X \mid Y) \leq \sqrt{2^{-(\alpha+3)}}$. If $H$ is independent from $(X, Y)$, then

$$
\mathrm{CP}^{1 / 2}((H, H(X)) \mid Y) \leq \sqrt{2^{-(q-1)}} .
$$

Proof. We bound the value of $\mathrm{CP}^{1 / 2}((H, H(X)) \mid Y)$ as follows:

$$
\begin{array}{ll}
\mathrm{CP}^{1 / 2}(H, H(X) \mid Y) & \\
=\underset{y \leftarrow Y}{\mathrm{E}}\left[\mathrm{CP}^{1 / 2}\left(H,\left.H(X)\right|_{Y=y}\right)\right] & \\
\leq \underset{y \leftarrow Y}{\mathrm{E}}\left[\mathrm{CP}^{1 / 2}(H) \cdot \sqrt{\mathrm{CP}\left(\left.X\right|_{Y=y}\right)+2^{-\alpha}}\right] & \left(\begin{array}{c}
\text { since } \mathrm{CP}(H, H(Z)) \leq \\
\mathrm{CP}(H) \cdot\left(\mathrm{CP}(Z)+2^{-\alpha}\right)
\end{array}\right) \\
\leq \underset{y \leftarrow Y}{\mathrm{E}}\left[\mathrm{CP}^{1 / 2}(H) \cdot\left(\mathrm{CP}^{1 / 2}\left(\left.X\right|_{Y=y}\right)+\sqrt{2^{-\alpha}}\right)\right] & (\text { Cauchy-Schwarz/Jensen })
\end{array}
$$




$$
\begin{aligned}
& =\mathrm{CP}^{1 / 2}(H) \cdot\left(\left(\underset{y \leftarrow Y}{\mathrm{E}}\left[\mathrm{CP}^{1 / 2}\left(\left.X\right|_{Y=y}\right)\right]\right)+\sqrt{2^{-\alpha}}\right) \\
& =\mathrm{CP}^{1 / 2}(H) \cdot\left(\mathrm{CP}^{1 / 2}(X \mid Y)+\sqrt{2^{-\alpha}}\right) \\
& =\sqrt{2^{-(q-\alpha)}} \cdot\left(\mathrm{CP}^{1 / 2}(X \mid Y)+\sqrt{2^{-\alpha}}\right) \\
& \leq \sqrt{2^{-(q-\alpha)}} \cdot\left(\sqrt{\frac{2^{-\alpha}}{8}}+\sqrt{2^{-\alpha}}\right) \\
& <\sqrt{2^{-(q-\alpha)}} \cdot\left(\sqrt{2^{-\alpha}} \cdot \sqrt{2}\right) \\
& =\sqrt{2^{-(q-1)}} \cdot \quad
\end{aligned}
$$

Restatement of Lemma 6.7. For any triple of (possibly correlated) random variables $X, Y$, and $Z$,

$$
\mathrm{CP}^{1 / 2}(X \mid Y) \leq \mathrm{CP}^{1 / 2}(X \mid(Y, Z)) \leq \sqrt{|\operatorname{Supp}(Z)|} \cdot \mathrm{CP}^{1 / 2}(X \mid Y) .
$$

Proof. For each $y \in \operatorname{Supp}(Y)$ and $z \in \operatorname{Supp}(Z)$, let $v_{y, z} \in \mathbb{R}^{\operatorname{Supp}(X)}$ be the vector whose $x$ th entry is $v_{y, z}(x)=\operatorname{Pr}[X=x \wedge Z=z \mid Y=y]$. With this, we compute for each $y$

$$
\begin{array}{rlr}
\left\|\sum_{z} v_{y, z}\right\|_{2} & \leq \sum_{z}\left\|v_{y, z}\right\|_{2} & \\
& \leq \sqrt{\operatorname{Supp}(Z)} \cdot\left(\sum_{z}\left\|v_{y, z}\right\|_{2}^{2}\right)^{1 / 2} & \text { (triangle inequality) } \\
& =\sqrt{\operatorname{Supp}(Z)} \cdot\left(\sum_{z, x} v_{y, z}(x)^{2}\right)^{1 / 2} \\
& \left.\leq \sqrt{\operatorname{Supp}(Z)} \cdot\left(\sum_{x}\left(\sum_{z} v_{y, z}(x)\right)^{2}\right)^{1 / 2} \quad \text { (nonnegativity of } v_{y, z}(x)\right) \\
& =\sqrt{\operatorname{Supp}(Z)} \cdot\left\|\sum_{z} v_{y, z}\right\|_{2}
\end{array}
$$

Since $\mathrm{CP}^{1 / 2}\left(\left.X\right|_{Y=y}\right)=\left\|\sum_{z} v_{y, z}\right\|_{2}$ and $\mathrm{CP}^{1 / 2}\left(\left(\left.X\right|_{Y=y}\right) \mid\left(\left.Z\right|_{Y=y}\right)\right)=\sum_{z}\left\|v_{y, z}\right\|_{2}$, taking expectations over $Y$ for both sides of the above inequalities yields the claim.

RESTATEMENT OF LEMMA 6.8. Let random variable $H$ denote a random hash function from a family of pairwise-independent hash functions $\mathcal{H}$ with range $\{0,1\}^{\alpha}$. For any $\varepsilon>0$, if $\mathrm{CP}(X) \leq \varepsilon^{2} \cdot 2^{-\alpha}$ and $H$ is independent from $X$, then random variable $(H, H(X))$ is $\varepsilon$-close in statistical distance to uniform.

Proof. Let $D=2^{q-\alpha}$ and $L=2^{\alpha}$. We bound the statistical distance of $(H, H(X))$ from uniform as follows:

$$
\begin{aligned}
\frac{1}{2}\left|(H, H(X))-U_{q}\right|_{1} & \leq \frac{\sqrt{D L}}{2}\left\|(H, H(X))-U_{q}\right\|_{2} \\
& =\frac{\sqrt{D L}}{2} \cdot \sqrt{\mathrm{CP}(H, H(X))-2^{-q}} \\
& \leq \frac{\sqrt{D L}}{2} \cdot \sqrt{\frac{1}{D}\left(\mathrm{CP}(X)+\frac{1}{L}\right)-\frac{1}{D L}}
\end{aligned}
$$




$$
\begin{aligned}
& =\frac{\sqrt{\mathrm{CP}(X) \cdot L}}{2} \\
& \leq \frac{\varepsilon}{2} . \quad \square
\end{aligned}
$$

Acknowledgments. We are grateful to the anonymous referees for their extensive comments and corrections. We also thank Oded Goldreich for helpful suggestions.

\section{REFERENCES}

[1] M. Nguyen, S. J. Ong, And S. Vadhan, Statistical zero-knowledge arguments for NP from any one-way function, in Proceedings of the 47th Annual Symposium on Foundations of Computer Science (FOCS), IEEE Computer Society, Washington, DC, 2006, pp. 3-14.

[2] I. Haitner And O. Reingold, Statistically-hiding commitment from any one-way function, in Proceedings of the 39th Annual ACM Symposium on Theory of Computing (STOC), ACM, New York, 2007.

[3] C. Shannon, Communication theory of secrecy systems, Bell System Tech. J., 28 (1949), pp. 656-715.

[4] W. Diffie and M. E. Hellman, New directions in cryptography, IEEE Trans. Inform. Theory, 22 (1976), pp. 644-654

[5] J. Håstad, R. Impagliazzo, L. A. Levin, And M. Luby, A pseudorandom generator from any one-way function, SIAM J. Comput., 28 (1999), pp. 1364-1396.

[6] O. Goldreich, S. Goldwasser, and S. Micali, How to construct random functions, J. ACM, 33 (1986), pp. 792-807.

[7] J. ROMPEL, One-way functions are necessary and sufficient for secure signatures, in Proceedings of the 22nd Annual ACM Symposium on Theory of Computing (STOC), ACM, New York, 1990, pp. 387-394.

[8] M. NAOR, Bit commitment using pseudorandomness, J. Cryptology, 4 (1991), pp. 151-158.

[9] O. Goldreich, S. Micali, And A. Wigderson, Proofs that yield nothing but their validity or all languages in NP have zero-knowledge proof systems, J. ACM, 38 (1991), pp. 691-729.

[10] R. Impagliazzo AND M. LuBy, One-way functions are essential for complexity based cryptography, in Proceedings of the 30th Annual Symposium on Foundations of Computer Science (FOCS), IEEE Computer Society, Washington, DC, 1989, pp. 230-235.

[11] R. Ostrovsky And A. Wigderson, One-way functions are essential for non-trivial zeroknowledge, in Proceedings of the 2nd Israel Symposium on Theory of Computing Systems, IEEE Computer Society, Washington, DC, 1993, pp. 3-17.

[12] R. ImPAGLIAZZO AND S. RUDICH, Limits on the provable consequences of one-way permutations, in Proceedings of the 21st Annual ACM Symposium on Theory of Computing (STOC), ACM, New York, 1989, pp. 44-61.

[13] D. Simon, Finding collisions on a one-way street: Can secure hash functions be based on general assumptions?, in Proceedings of Advances in Cryptology-EUROCRYPT '98, Lecture Notes in Comput. Sci. 1403, Springer-Verlag, New York, 1998, pp. 334-345.

[14] G. Brassard, D. Chaum, and C. Crépeau, Minimum disclosure proofs of knowledge, J. Comput. System Sci., 37 (1988), pp. 156-189.

[15] G. Brassard, C. CRéPeau, AND M. Yung, Constant-round perfect zero-knowledge computationally convincing protocols, Theoret. Comput. Sci., 84 (1991), pp. 23-52.

[16] M. Naor, R. Ostrovsky, R. Venkatesan, and M. Yung, Perfect zero-knowledge arguments for NP using any one-way permutation, J. Cryptology, 11 (1998), pp. 87-108.

[17] O. Goldreich, S. Micali, and A. Wigderson, How to play any mental game or a completeness theorem for protocols with honest majority, in Proceedings of the 19th Annual ACM Symposium on Theory of Computing (STOC), ACM, New York, 1987, pp. 218-229.

[18] J. F. Boyar, S. A. Kurtz, and M. W. Krentel, A discrete logarithm implementation of perfect zero-knowledge blobs, J. Cryptology, 2 (1990), pp. 63-76.

[19] D. Chaum, I. Damgård, And J. GraAF, Multiparty computations ensuring privacy of each party's input and correctness of the result, in Proceedings of Advances in CryptologyCRYPTO '87, Lecture Notes in Comput. Sci. 293, Springer-Verlag, New York, 1987, pp. 87119.

[20] T. P. Pedersen, Non-interactive and information-theoretic secure verifiable secret sharing, in Proceedings of Advances in Cryptology-CRYPTO '91, Lecture Notes in Comput. Sci. 576, Springer-Verlag, New York, 1991, pp. 129-140. 
[21] S. Goldwasser, S. Micali, and R. L. Rivest, A digital signature scheme secure against adaptive chosen-message attacks, SIAM J. Computing, 17 (1988), pp. 281-308.

[22] O. Goldreich AND A. KAHAN, How to construct constant-round zero-knowledge proof systems for NP, J. Cryptology, 9 (1996), pp. 167-190.

[23] M. NAOR AND M. YUng, Universal one-way hash functions and their cryptographic applications, in Proceedings of the 21st Annual ACM Symposium on Theory of Computing (STOC), ACM, New York, 1989, pp. 33-43.

[24] I. B. Damgård, T. P. Pedersen, and B. Pfitzmann, Statistical secrecy and multibit commitments, IEEE Trans. Inform. Theory, 44 (1998), pp. 1143-1151.

[25] I. Haitner, O. Horvitz, J. Katz, C. Koo, R. Morselli, and R. Shaltiel, Reducing complexity assumptions for statistically-hiding commitment, in Proceedings of Advances in Cryptology-EUROCRYPT 2005, Lecture Notes in Comput. Sci. 3494, Springer-Verlag, New York, 2005, pp. 58-77.

[26] S. J. ONG AND S. VADHAN, Zero knowledge and soundness are symmetric, in Advances in Cryptology-EUROCRYPT 2007, Lecture Notes in Comput. Sci. 4515, Springer-Verlag, New York, 2007, pp. 187-209.

[27] R. Ostrovsky, One-way functions, hard on average problems, and statistical zero-knowledge proofs, in Proceedings of the 6th Annual Structure in Complexity Theory Conference, IEEE Computer Society, Washington, DC, 1991, pp. 133-138.

[28] M. NGUYen And S. VAdhan, Zero knowledge with efficient provers, in Proceedings of the 38th Annual ACM Symposium on Theory of Computing (STOC), ACM, New York, 2006, pp. 287-295.

[29] J. Katz And C. Koo, On Constructing Universal One-Way Hash Functions from Arbitrary One-Way Functions, Cryptology ePrint Archive, http://eprint.iacr.org/2005/328, 2005.

[30] I. Haitner, O. Reingold, S. Vadhan, and H. Wee, Inaccessible entropy, in Proceedings of the 41st Annual ACM Symposium on Theory of Computing (STOC), ACM, New York, to appear.

[31] O. Goldreich, Foundations of Cryptography: Basic Tools, Cambridge University Press, Cambridge, UK, 2001.

[32] R. Ostrovsky, R. Venkatesan, and M. Yung, Fair games against an all-powerful adversary, in Advances in Computational Complexity Theory, DIMACS Ser. Discrete Math. Theoret. Comput. Sci. 13, AMS, Providence, RI, 1993, pp. 155-169.

[33] I. Haitner and O. Reingold, A new interactive hashing theorem, in Proceedings of the 22nd Annual IEEE Conference on Computational Complexity, IEEE Computer Society, Washington, DC, 2007, pp. 319-332.

[34] C. Cachin, C. Crépeau, and J. Marcil, Oblivious transfer with a memory-bounded receiver, in Proceedings of the 39th Annual Symposium on Foundations of Computer Science (FOCS), IEEE Computer Society, Washington, DC, 1998, pp. 493-502.

[35] Y. Z. Ding, D. Harnik, A. Rosen, and R. Shaltiel, Constant-round oblivious transfer in the bounded storage model, in Theory of Cryptography, First Theory of Cryptography Conference, TCC 2004, Lecture Notes in Comput. Sci. 2951, Springer-Verlag, New York, 2004, pp. 446-472.

[36] C. CRÉPEAU AND G. SAVvides, Optimal reductions between oblivious transfers using interactive hashing, in Proceedings of Advances in Cryptology-EUROCRYPT 2006, Lecture Notes in Comput. Sci. 4004, Springer-Verlag, New York, 2006, pp. 201-221.

[37] C. H. Bennett, G. Brassard, and J.-M. Robert, Privacy amplification by public discussion, SIAM J. Comput., 17 (1988), pp. 210-229.

[38] O. Goldreich, S. Goldwasser, and N. Linial, Fault-tolerant computation in the full information model, SIAM J. Comput., 27 (1998), pp. 506-544.

[39] S. J. Ong And S. Vadhan, An equivalence between zero knowledge and commitments, in Proceedings of the Third Theory of Cryptography Conference (TCC '08), R. Canetti, ed., Lecture Notes in Comput. Sci. 4948, Springer-Verlag, New York, 2008, pp. 482-500.

[40] O. Reingold, L. Trevisan, and S. Vadhan, Notions of reducibility between cryptographic primitives, in Proceedings of the First Theory of Cryptography Conference (TCC '04), M. Naor, ed., Lecture Notes in Comput. Sci. 2951, Springer-Verlag, New York, 2004, pp. 1-20. 